\title{
FLOER COHOMOLOGY, MULTIPLICITY AND THE LOG CANONICAL THRESHOLD.
}

\author{
MARK MCLEAN
}

\begin{abstract}
Let $f$ be a polynomial over the complex numbers with an isolated singularity at 0 . We show that the multiplicity and the log canonical threshold of $f$ at 0 are invariants of the link of $f$ viewed as a contact submanifold of the sphere.

This is done by first constructing a spectral sequence converging to the fixed point Floer cohomology of any iterate of the Milnor monodromy map whose $E^{1}$ page is explicitly described in terms of a $\log$ resolution of $f$. This spectral sequence is a generalization of a formula by A'Campo. By looking at this spectral sequence, we get a purely Floer theoretic description of the multiplicity and $\log$ canonical threshold of $f$.
\end{abstract}

\section{Contents}

1. Introduction

1.1. Sketch of the proof of Theorem 1.2

1.2. Plan of the paper

1.3. Conventions

2. Multiplicities and Discrepancies of Exceptional Divisors.

3. Liouville domains, Symplectomorphisms and Open Books

4. Fixed Point Floer Cohomology Definition

5. Constructing a Well Behaved Contact Open Book.

5.1. Some Preliminary Definitions

5.2. Trivializing Line Bundles

5.3. Links of Divisors and Open Books

5.4. Constructing a Contact Open Book from a Model Resolution

5.5. Dynamics of abstract contact open books associated to model resolutions.

6. Proof of Theorem 1.2 and Corollary 1.3 .

7. Appendix A: Gradings and Canonical Bundles

8. Appendix B: Contactomorphisms of Mapping Tori and Floer Cohomology

9. Appendix C: A Morse-Bott Spectral Sequence

References

\section{INTRODUCTION}

Let $f: \mathbb{C}^{n+1} \rightarrow \mathbb{C}$ be a polynomial with an isolated singular point at 0 where $n \geq$ 1. Let $S_{\epsilon} \subset \mathbb{C}^{n+1}$ be the sphere of radius $\epsilon$ centered at 0 . The link of $f$ at 0 is the submanifold $L_{f} \equiv f^{-1}(0) \cap S_{\epsilon} \subset S_{\epsilon}$ where $\epsilon>0$ is sufficiently small. One can ask the following question: what is the relationship between the link of $f$ and various algebraic properties of $f$ ? For instance Zariski in [Zar72] asked whether the multiplicity of $f$ at 0 depends only on the embedding $L_{f} \subset S_{\epsilon}$. Another important invariant is the log canonical 
threshold (see Ati70, Mus12 or Definition 2.1). Again one can ask if this is an invariant of $L_{f} \subset S_{\epsilon}$ (see [Bud12, Section 1.6]). We will answer weaker versions of these questions. If $\epsilon>0$ is small enough, it turns out that $L_{f}$ is naturally a contact submanifold of $S_{\epsilon}$ (See [Var82]). If $g: \mathbb{C}^{n+1} \longrightarrow \mathbb{C}$ is another polynomial with isolated singularity at 0 then we say that $f$ and $g$ have embedded contactomorphic links if there is a contactomorphism $\Phi: S_{\epsilon} \longrightarrow S_{\epsilon}$ sending $L_{f}$ to $L_{g}$. Varchenko showed in Var82] that if there is a holomorphic change of coordinates sending $f$ to $g$ then they have embedded contactomorphic links. One of the goals of this paper is to prove the following theorem.

Theorem 1.1. Suppose that $f, g: \mathbb{C}^{n+1} \longrightarrow \mathbb{C}$ are two polynomials with isolated singular points at 0 with embedded contactomorphic links. Then the multiplicity and the log canonical threshold of $f$ and $g$ are equal.

We will prove this theorem by finding formulas for the multiplicity and log canonical threshold in terms of a sequence Floer cohomology groups. The key technical result of this paper proving the above theorem will be a natural generalization of a formula by A'Campo A'C75.

For all $\epsilon>0$ small enough, there is a smooth fibration

$$
\arg (f): S_{\epsilon}-f^{-1}(0) \longrightarrow \mathbb{R} / 2 \pi \mathbb{Z}, \quad \arg (f)(z) \equiv \arg (f(z))
$$

called the Milnor fibration associated to $f$ (see [Mil68, Chapter 4]). A fiber $M_{f} \equiv \arg (f)^{-1}(0)$ is called the Milnor fiber of $f$. By choosing an appropriate connection on this fibration, there is a natural compactly supported diffeomorphism $\phi: M_{f} \longrightarrow M_{f}$ given by parallel transporting around the circle $\mathbb{R} / 2 \pi \mathbb{Z}$ called the Milnor monodromy map. The Lefschetz number $\Lambda\left(\phi^{m}\right)$ of $\phi^{m}$ is defined to be

$$
\Lambda\left(\phi^{m}\right) \equiv \sum_{j=0}^{\infty}(-1)^{j} \operatorname{Tr}\left(\phi_{*}^{m}: H_{j}\left(M_{f} ; \mathbb{Z}\right) \longrightarrow H_{j}\left(M_{f} ; \mathbb{Z}\right)\right),
$$

and this is an invariant of the embedding $L_{f} \subset S_{\epsilon}$ for each $m>0$. A'Campo in A'C75] computed these numbers in the following way. Let $\pi: Y \longrightarrow \mathbb{C}^{n+1}$ be a log resolution of the pair $\left(\mathbb{C}^{n+1}, f^{-1}(0)\right)$ at 0 . Let $\left(E_{j}\right)_{j \in \check{S}}$ be the prime exceptional divisors of this resolution and define $E_{\star_{S}}$ to be the proper transform $\overline{\pi^{-1}\left(f^{-1}(0)-0\right)}$ of $f^{-1}(0)$. Let $S \equiv \check{S} \sqcup\left\{\star_{S}\right\}$. Define $E_{j}^{0} \equiv E_{j}-\cup_{i \in S-\{j\}} E_{i}$ for all $j \in S$ and define $S_{m} \equiv\left\{i \in \check{S}: \operatorname{ord}_{f}\left(E_{i}\right)\right.$ divides $\left.m\right\}$ for all $m>0$. A'Campo showed that

$$
\Lambda\left(\phi^{m}\right)=\sum_{i \in S_{m}} \operatorname{ord}_{f}\left(E_{i}\right) \chi\left(E_{i}^{o}\right), \quad \forall m>0 .
$$

The key technical result of this paper (Theorem 1.2) is a spectral sequence converging to a group whose Euler characteristic is naturally equal to the left hand side of (1.1) multiplied by $(-1)^{n}$ and so that the Euler characteristic of the $E^{1}$ page is naturally equal to the right had side multiplied by $(-1)^{n}$. We will now explain this result.

For $\epsilon>0$ small enough, the Milnor fiber $M_{f}$ is naturally a symplectic manifold and $\phi$ can be made to be a compactly supported symplectomorphism (see Section 3). For any compactly supported symplectomorphism $\psi$ satisfying some additional properties, one can assign a group $H F^{*}(\psi,+)$ called the Floer cohomology group of $\psi$ (see [Sei01, Section 4] or Section 4 of this paper). The Euler characteristic of this group is $(-1)^{n}$ multiplied by the Lefschetz number of $\psi$ (See property (HF1) in Section 4 of this paper). As a result, we have a sequence of groups $H F^{*}\left(\phi^{m},+\right)$ whose Euler characteristic is $(-1)^{n} \Lambda\left(\phi^{m}\right)$ for all $m>0$. All of these groups are invariants of the link of $f$ up to embedded contactomorphism (See Lemma 
8.17). The $\log$ resolution $\pi: Y \longrightarrow \mathbb{C}^{n+1}$ is called a multiplicity $m$ separating resolution if $\operatorname{ord}_{f}\left(E_{i}\right)+\operatorname{ord}_{f}\left(E_{j}\right)>m$ for all $i, j \in S$ satisfying $i \neq j$ and $E_{i} \cap E_{j} \neq \emptyset$.

Theorem 1.2. Suppose that $\pi: Y \longrightarrow \mathbb{C}^{n+1}$ is a multiplicity $m$ separating resolution for some $m \in \mathbb{N}_{>0}$. Let $\left(w_{i}\right)_{i \in \check{S}}$ be positive integers so that $-\sum_{i \in \check{S}} w_{i} E_{i}$ is ample. Let $a_{i}$ be the discrepancy of $E_{i}$ (see Definition 2.1) and define $k_{i} \equiv \frac{m}{\operatorname{ord}_{f}\left(E_{i}\right)}$ for all $i \in S_{m}$. Then there is a cohomological spectral sequence converging to $H F^{*}\left(\phi^{m},+\right)$ with $E^{1}$ page

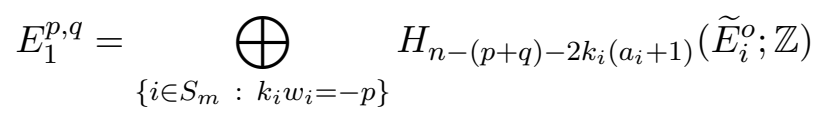

where $\widetilde{E}_{i}^{o}$ is an $m_{i}$-fold cover of $E_{i}^{o}$ for all $i \in S_{m}$. The cover $\widetilde{E}_{i}^{o}$ is constructed as follows: Let $U_{i}$ be a neighborhood of $E_{i}^{o}$ inside $Y-\cup_{j \in S-i} E_{i}$ which deformation retracts on to $E_{i}$, let $\iota_{i}: U_{i}-E_{i}^{o} \longrightarrow U_{i}$ be the natural inclusion map and define

$$
f_{i}: U_{i}-E_{i}^{o} \longrightarrow \mathbb{C}^{*}, f_{i}(x) \equiv f(\pi(x)) .
$$

Then $\widetilde{E}_{i}^{o}$ is disjoint union of connected covers corresponding to normal subgroup

$$
G_{i}:=\left(\iota_{i}\right)_{*}\left(\operatorname{ker}\left(\left(f_{i}\right)_{*}\right)\right) \subset \pi_{1}\left(U_{i}\right)=\pi_{1}\left(E_{i}^{o}\right)
$$

and the number of such covers is $m_{i}$ divided by the index of $G_{i}$ in $\pi_{1}\left(E_{i}^{o}\right)$.

The covers $\widetilde{E}_{i}^{o}$ are described in an explicit algebraic way in [DL02, Section 2.3]. Intuitively, we should think of $\widetilde{E}_{i}^{o}$ in the following way: if $U_{i}$ was a 'nice' tubular neighborhood of $E_{i}^{o}$ and we had a 'nice' projection map $Q_{i}: U_{i} \longrightarrow E_{i}^{o}$ then $Q_{i}:(\pi \circ f)^{-1}(\epsilon) \cap U_{i} \longrightarrow E_{i}^{o}$ would be a covering map on to its image homotopic to $\widetilde{E}_{i}^{o}$ for $\epsilon>0$ small enough (see the proof of Lemma 5.40).

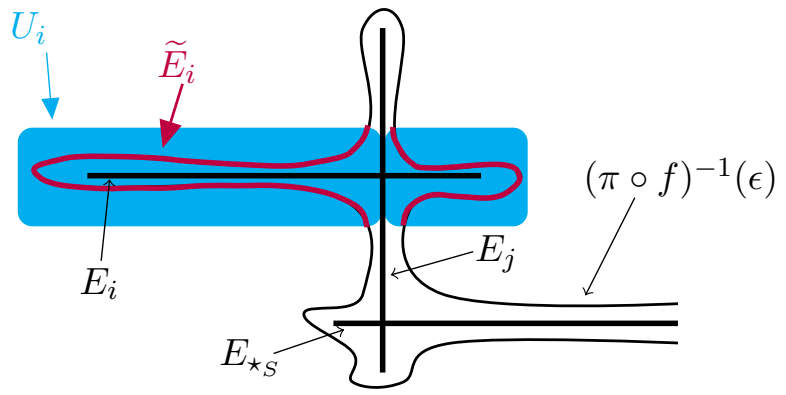

By Lemma 2.4 combined with [Hir64], a resolution satisfying the properties stated in the above Theorem exists for each $m>0$. By looking at this spectral sequence above, one gets the following corollary:

Corollary 1.3. For each $m>0$, define $\nu_{m} \equiv \sup \left\{\alpha: H F^{\alpha}\left(\phi^{m},+\right) \neq 0\right\}$ and define $\mu_{m} \equiv$ $\inf \left\{k_{i}\left(a_{i}+1\right): i \in S_{m}\right\}$ where $k_{i}$ and $a_{i}$ are defined as in Theorem 1.2 above. Then

$$
\nu_{m}=n-2 \mu_{m} \quad \forall m>0 .
$$

In particular $H F^{*}\left(\phi^{m},+\right)$ vanishes if and only if $\mu_{m}=\infty$. Also the numbers $\mu_{m}$ are invariants of the link up to embedded contactomorphism since the groups $H F^{*}\left(\phi^{m},+\right)$ are.

We will prove this corollary in Section 6. We have an immediate corollary of this corollary proving a conjecture of Seidel [Sei14] regarding the multiplicity of a singularity. 
Corollary 1.4. The multiplicity of $f$ is the smallest $m>0$ so that $H F^{*}\left(\phi^{m},+\right) \neq 0$. The log canonical threshold of $f$ at 0 is

$$
l c t_{0}(f)=\liminf _{m \rightarrow \infty}\left(\inf \left\{-\frac{\alpha}{2 m}: H F^{\alpha}\left(\phi^{m},+\right) \neq 0 \text { or }-\frac{\alpha}{2 m}=1\right\}\right) .
$$

Since $H F^{*}\left(\phi^{m},+\right)$ is an invariant of the link of $f$ up to embedded contactomorphism by Lemma 8.17 we get that Theorem 1.1 follows immediately from Corollary 1.4 .

For each $m>0$, Denef and Loeser in DL02 constructed natural spaces $\chi_{m, 1}$ whose Euler characteristic is $\Lambda\left(\phi^{m}\right)$. Therefore it is natural to ask, what is the relationship between these spaces and the groups $H F^{*}\left(\phi^{m},+\right)$, if any? Such a question was considered by Seidel (see DL02, Remark 2.7]). It might be interesting to see if there is a similar spectral sequence converging to $H_{*}\left(\chi_{m, 1} ; \mathbb{Z}\right)$ since these spaces admit a natural stratification induced by the strata of the log resolution $\pi$. A possible proof would exploit the spectral sequence Pet16, Formula (3)] combined with [DL02, Lemma 2.2] (see also the calculations in the proof of [DL02, Lemma 2.5]).

1.1. Sketch of the proof of Theorem 1.2. We will now state one of the key properties of the group $H F^{*}(\psi,+)$ that will be used in this proof. This property is stated precisely in (HF3) Section 4 and proven in Appendix C.

Spectral Sequence Property: Suppose that the set of fixed points of $\psi$ is a disjoint union of connected codimension 0 submanifolds $B_{1}, \cdots, B_{l}$ with boundary and corners and suppose that $\psi$ behaves in a particular way near the boundary of $B_{i}$ for each $i$. Then there is a grading $C Z\left(B_{i}\right) \in \mathbb{Z}$ for each $B_{i}$ and there is a specific function $\iota:\{1, \cdots, l\} \longrightarrow \mathbb{N}$ so that there is a spectral sequence converging to $H F^{*}(\psi,+)$ with $E^{1}$ page equal to

$$
E_{1}^{p, q}=\bigoplus_{\{i \in\{1, \cdots, l\}: \iota(i)=p\}} H_{n-(p+q)-C Z\left(\phi, B_{i}\right)}\left(B_{i} ; \mathbb{Z}\right)
$$

The spectral sequence above is an example of a Morse-Bott spectral sequence (See [Bot57, Corollary 2] and [Hut02, Section 6.4] for other similar examples). Therefore in order to prove Theorem 1.2 it would be sufficient for us to deform the monodromy symplectomorphism $\phi^{m}$ so that the set of fixed points is a union of codimension 0 submanifolds homotopic to $\widetilde{E}_{i_{p}}^{o}$ for each $i \in\{1, \cdots, l\}$. The problem is that we cannot quite do this, but we can construct a new symplectomorphism with the required fixed point sets without changing $H F^{*}\left(\phi^{m},+\right)$. Also, Theorem 1.2 really requires a specific ordering of the submanifolds $\widetilde{E}_{i_{p}}^{o}$ corresponding to the sequence of positive integers $\left(w_{j}\right)_{j \in S}$ but we will ignore this detail here, as the main applications of this paper do not need such an ordering. We will now explain how to modify $\phi^{m}$ without changing $H F^{*}\left(\phi^{m},+\right)$ so that it has this fixed point property. This is done in Section 5 .

We have a natural symplectic form $\omega_{Y}$ on $Y$ coming from the ample divisor $-\sum_{i \in S} w_{i} E_{i}$. This symplectic form gives us a natural Ehresmann connection on $\pi^{*} f$ away form $\left(\pi^{*} f\right)^{-1}(0)$ and hence gives us a monodromy map. First of all, we deform $\omega_{Y}$ so that it behaves well with respect to $\pi^{*} f$ (See Sections 5.1 and 5.2 ). The key idea is that since $\pi^{*} f$ locally looks like $\prod_{i=1}^{m} z_{i}^{m}$, we can deform $\omega_{Y}$ so that it basically looks like the standard symplectic form in these local charts (with a few modifications). The next step is to show that the corresponding monodromy map $\psi$ satisfies $H F^{*}\left(\psi^{m},+\right)=H F^{*}\left(\phi^{m},+\right.$ ) (See sections 5.3, 5.4 and Appendix A). Here we are using the fact that these Floer cohomology groups are invariants of the mapping tori of $\phi^{m}$ and $\psi^{m}$ respectively along with an additional contact structure on these tori and some additional data. Finally we need to compute the fixed points of the monodromy map so that we can apply our spectral sequence property (See section 5.5). 
1.2. Plan of the paper. In Section 2 we construct algebraic invariants of $\left(\mathbb{C}^{n+1}, f^{-1}(0)\right)$ which will be used to tell us the smallest non-vanishing degree of $H F^{*}\left(\phi^{m},+\right)$ for each $m$. These invariants are constructed by looking at the multiplicities and discrepancies of the prime exceptional divisors $\left(E_{i}\right)_{i \in S}$ of a resolution.

In Section 3 we give some basic definitions of the main objects in symplectic and contact geometry that will be used in this paper. These include Liouville domains, (abstract) open books, contact mapping cylinders and gradings. In Section 4 we give a definition Floer cohomology of a symplectomorphism. We also state the three main properties (HF1)-(HF3) of the Floer cohomology group $H F^{*}(\psi,+)$ that will be needed for this paper. These properties will be proven in Appendix B and C.

Section 5 is the largest section of the paper. This section is used to construct a monodromy symplectomorphism nice enough so that we can use the properties from Section 4 to prove Theorem 1.2. This section heavily relies on results and notation from MTZ14a. Section 6 contains a proof of Theorem 1.2 and Corollary 1.3 .

Appendix $\mathrm{A}$ is deals with gradings. It enables us to compute the quantities $C Z\left(B_{i}\right)$ stated in the spectral sequence property in the sketch of the proof of Theorem 1.2 earlier. Appendix B proves that the groups $H F^{*}\left(\phi^{m},+\right)$ only depend on the link $L_{f} \subset S_{\epsilon}$ as a contact submanifold. This relies heavily on results of [McL12b]. In Appendix $\mathrm{C}$ we prove the spectral sequence property of $H F^{*}(\psi,+)$ described above.

1.3. Conventions. If $(M, \omega)$ is a symplectic manifold and $\theta$ is a 1-form then its $\omega$-dual $X_{\theta}^{\omega}$ is the unique vector field satisfying $\omega\left(X_{\theta}^{\omega}, Y\right)=\theta(Y)$ for all vectors $Y$. Sometimes we just write $X_{\theta}$ instead of $X_{\theta}^{\omega}$ if it is clear from the context that the symplectic form we are using is $\omega$. For a smooth function $H: M \longrightarrow \mathbb{R}$, we define $X_{H} \equiv X_{-d H}$. The time $t$ flow of $X_{-d H}$ will be denoted by $\phi_{t}^{H}: M \longrightarrow M$ (this is called the time $t$ Hamiltonian flow of $H$ ).

Also if $f: B^{\prime} \longrightarrow B$ is a smooth map and $\pi: V \longrightarrow B$ is a vector bundle then we will write elements of the pullback bundle $f^{*}(V)$ as pairs $\left(b^{\prime}, v\right) \in B^{\prime} \times V$ satisfying $f\left(b^{\prime}\right)=\pi(v)$. For any fiber bundle $\pi: E \longrightarrow B$ and any subsets $N \subset E, C \subset B$, we define $\left.N\right|_{C} \equiv N \cap \pi^{-1}(C)$. To avoid cluttered notation, we will not distinguish between an element of a set and a subset of size 1 when the context is clear (e.g. $i$ will quite often mean $\{i\}$ ). We also write $\operatorname{Dom}(f)$ and $\operatorname{Im}(f)$ for the domain and image of a map $f$. For any set $I$, we define $\mathbb{N}_{>0}^{I}$ to be the set of tuples $\left(k_{i}\right)_{i \in I}$ where $k_{i} \in \mathbb{N}_{>0}$.

Acknowledgments: Many thanks to Mircea Mustaţă for suggesting the connection with log canonical threshold. Also many thanks to Paul Seidel for answering some of my questions. This paper is supported by the NSF grant DMS-1508207.

\section{Multiplicities and Discrepancies of Exceptional Divisors.}

In this section we will introduce some of the basic tools that are needed from algebraic geometry. We will define the multiplicity and log canonical threshold of an isolated hypersurface singularity as well as some more general invariants. We will also explain how to compute these invariants in terms of certain resolutions called multiplicity $m$ separating resolutions and show how such computational techniques do not depend on the choice of resolution.

Let $f: \mathbb{C}^{n+1} \rightarrow \mathbb{C}$ be a polynomial with an isolated singular point at 0 .

Definition 2.1. A $\log$ resolution at 0 of the pair $\left(\mathbb{C}^{n+1}, f^{-1}(0)\right)$ is a proper holomorphic map $\pi: Y \rightarrow \mathbb{C}^{n+1}$ from a complex manifold $Y$ so that there is some open set $U \subset \mathbb{C}^{n+1}$ containing 0 satisfying: 
(1) $\pi^{-1}\left(f^{-1}(0)\right) \cap U$ is a finite union of smooth transversally intersecting hypersurfaces $\left(E_{i}\right)_{i \in S}$. We will call such divisors resolution divisors. Each $E_{j}$ satisfying $\pi\left(E_{j}\right)=\{0\}$ is called a prime exceptional divisor. We require that the prime exceptional divisors are connected. We also require that there is a unique element $\star_{S} \in S$ where $E_{\star_{S}}=$ $\overline{\pi^{-1}\left(f^{-1}(0)-0\right)}\left(E_{\star_{S}}\right.$ need not be connected). We call $E_{\star_{S}}$ the proper transform of $f^{-1}(0)$.

(2) $\left.\pi\right|_{\pi^{-1}(U \backslash\{0\})}: \pi^{-1}(U \backslash\{0\}) \rightarrow U \backslash\{0\}$ is a biholomorpihsm.

Since the only singularities in this paper will be at $0 \in \mathbb{C}^{n+1}$, we will just call a log resolution at 0 of $f$ a $\log$ resolution of $\left(\mathbb{C}^{n+1}, f^{-1}(0)\right)$.

The multiplicity of $f$ along $E_{j}$, denoted by $\operatorname{ord}_{f}\left(E_{j}\right)$, is the order of $\pi^{*} f$ along $E_{j}$. In other words, choose some local coordinate chart $z_{1}, \cdots, z_{n}$ centered at a generic point of $E_{j}$ so that $E_{j}=\left\{z_{1}=0\right\}$ and define $\operatorname{ord}_{f}(E) \equiv k$ where $k \in \mathbb{Z}$ satisfies $\pi^{*} f=g z_{1}^{k}$ in this coordinate system for some holomorphic function $g$ satisfying $g(0) \neq 0$.

The discrepancy of $E_{j}$ denoted by $a\left(E_{j}\right)$ is calculated as follows: choose local holomorphic coordinates $y_{1}, \cdots, y_{n}$ on $Y$ centered at a point on $E_{j}$ and holomorphic coordinates $x_{1}, \cdots, x_{n}$ on $\mathbb{C}^{n+1}$ centered at 0 . Then $a\left(E_{j}\right)$ is the order of the Jacobian determinant of $f$ along $E_{j}$ expressed in these coordinates. This quantity does not depend on the choices of such holomorphic coordinates. The multiplicity of $f$ at 0 is $\min \left\{\operatorname{ord}_{f}\left(E_{j}\right): j \in S-\star_{S}\right\}$ and the $\log$ canonical threshold is $\min \left\{\frac{a\left(E_{j}\right)+1}{\operatorname{ord}_{f}\left(E_{j}\right)}: j \in S\right\}$.

Throughout this paper, we will define $E_{I}$ to be $\cap_{j \in I} E_{j}$ for each $I \subset S$. If $I$ is the empty set then $E_{I}$ is the entire manifold $Y$.

Definition 2.2. Let $\pi: Y \rightarrow \mathbb{C}^{n+1}$ be a log resolution of $\left(\mathbb{C}^{n+1}, f^{-1}(0)\right)$ as above. For each $m \in \mathbb{N}_{>0}$, we define the minimal multiplicity $m$ discrepancy to be:

$\operatorname{md}_{m}(\pi, f) \equiv \inf \left\{\sum_{j \in I} k_{j} a\left(E_{j}\right): I \subset S, I \neq \star_{S},\left(k_{j}\right)_{j \in I} \in \mathbb{N}_{>0}^{I}, E_{I} \neq \emptyset, \sum_{j \in I} k_{j} \operatorname{ord}_{f}\left(E_{j}\right)=m\right\}$.

Our convention here is that infimum of the empty set is $\infty$. Later on in Lemma 2.6 we will show that $\operatorname{md}_{m}(\pi, f)$ does not depend on $\pi$ and hence we can define $\operatorname{md}_{m}(f) \equiv \operatorname{md}_{m}(\pi, f)$ for some choice of log resolution $\pi$.

A morphism $\pi: Y \rightarrow \mathbb{C}^{n+1}$ is a multiplicity $m$ separating resolution if it is a log resolution of $\left(\mathbb{C}^{n+1}, f^{-1}(0)\right)$ such that for any two resolution divisors $E$ and $F$ of $\pi$ satisfying $E \cap F \neq \emptyset$, the sum of the multiplicities of $f$ along $E$ and $F$ is greater than $m$.

Multiplicity $m$ separating resolutions make it much easier for us to compute the minimal multiplicity $m$ discrepancy.

Lemma 2.3. If $\pi: Y \rightarrow \mathbb{C}^{n+1}$ is a multiplicity $m$ separating resolution of $\left(\mathbb{C}^{n+1}, f^{-1}(0)\right)$ and $\left(E_{j}\right)_{j \in S}$ are its resolution divisors then

$$
m d_{m}(\pi, f)=\inf \left\{k a\left(E_{j}\right): k \in \mathbb{N}_{>0}, j \in S-\star_{S}, \operatorname{kord}_{f}\left(E_{j}\right)=m\right\} .
$$

Proof. This follows from the fact that if $\sum_{j \in I} k_{j} \operatorname{ord}_{f}\left(E_{j}\right)=m$ and $E_{I} \neq \emptyset$ for some $I \subset S-\star_{S}$ and $\left(k_{j}\right)_{j \in I} \in \mathbb{N}_{>0}^{I}$ then $|I|=1$.

Lemma 2.4. If we have any log resolution then we can blow such a resolution up along strata of $\cup_{i \in S} E_{i}$ inside $\pi^{-1}(0)$ so that it becomes a multiplicity $m$ separating resolution. 
Proof. Let $\pi: Y \longrightarrow \mathbb{C}^{n+1}$ be a resolution with resolution divisors $\left(E_{j}\right)_{j \in S}$. Define

$$
a_{Y} \equiv \min \left\{\sum_{j \in I} k_{j} \operatorname{ord}_{f}\left(E_{j}\right): I \subset S,|I|=2, E_{I} \neq \emptyset,\left(k_{j}\right)_{j \in I} \in \mathbb{N}_{>0}^{I}\right\} .
$$

Let $b_{Y}$ be the number of elements in the set

$$
B_{Y} \equiv\left\{I \subset S:|I|=2, E_{I} \neq \emptyset, \sum_{j \in I} k_{j} \operatorname{ord}_{f}\left(E_{j}\right)=a_{Y}\right\} .
$$

Since $b_{Y} \geq 1$, choose $I \in B_{Y}$. Let $Y^{\prime}$ be the blowup of $Y$ along $E_{I}$. Then $a_{Y}-b_{Y}$ is strictly smaller than $a_{Y^{\prime}}-b_{Y^{\prime}}$. Hence by induction we can blow up $Y$ along subsets of the form $E_{I}$ until we get a $\log$ resolution $\pi^{\prime \prime}: Y^{\prime \prime} \longrightarrow \mathbb{C}$ of $\left(\mathbb{C}^{n+1}, f^{-1}(0)\right)$ so that $a_{Y^{\prime \prime}}-b_{Y^{\prime \prime}} \geq m$. Since $b_{Y^{\prime \prime}} \geq 1$, we get that $a_{Y^{\prime \prime}}>m$. Hence $\pi^{\prime \prime}$ is a multiplicity $m$ separating resolution.

Lemma 2.5. Let $\pi: Y \rightarrow \mathbb{C}^{n+1}$ be a log resolution of $\left(\mathbb{C}^{n+1}, f^{-1}(0)\right)$ and $I \subset S$ a subset satisfying $|I| \geq 2$. Let $\check{\pi}: \check{Y} \rightarrow \mathbb{C}^{n+1}$ be the log resolution of $\left(\mathbb{C}^{n+1}, f^{-1}(0)\right)$ obtained by blowing up $Y$ along $E_{I}$. Then

$$
m d_{m}(\check{\pi}, f)=m d_{m}(\pi, f) .
$$

Proof. Let $\left(E_{j}\right)_{j \in S}$ be the resolutions divisors of $\pi$. Let $\check{E}_{j}$ be the proper transform of $E_{j}$ in $\check{Y}$ for all $j \in S$. Then

$$
a\left(\check{E}_{j}\right)=a\left(E_{j}\right) \text { and } \operatorname{ord}_{f}\left(\check{E}_{j}\right)=\operatorname{ord}_{f}\left(E_{j}\right) .
$$

Let $E$ be the exceptional divisor of the blowdown map $\check{Y} \longrightarrow Y$. Then by looking at a local model of the blowdown map and using the chain rule we get

$$
a(E)=|I|-1+\sum_{j \in I} a\left(E_{j}\right), \quad \operatorname{ord}_{f}(E)=\sum_{j \in I} \operatorname{ord}_{f}\left(E_{j}\right) .
$$

Suppose for some $\check{I} \subset S$ satisfying $E_{\check{I}} \neq \emptyset$, some $k \in \mathbb{N}_{\geq 0}$ and $\left(k_{j}\right)_{j \in \check{I}} \in \mathbb{N}_{>0}^{\check{I}}$ we have

$$
k \operatorname{ord}_{f}(E)+\sum_{j \in \check{I}} k_{j} \operatorname{ord}_{f}\left(\check{E}_{j}\right)=m .
$$

Then by Equations (2.1) and (2.2) we have

$$
k a(E)+\sum_{j \in \check{I}} k_{j} a\left(\check{E}_{j}\right)=k(|I|-1)+\sum_{j \in I-\check{I}} k a\left(E_{j}\right)+\sum_{j \in I \cap I^{\prime}}\left(k+k_{j}\right) a\left(E_{j}\right)+\sum_{j \in \check{I}-I} k_{j} a\left(E_{j}\right) .
$$

Also Equations (2.1) and (2.2) tell us that

$$
\begin{array}{r}
\operatorname{kord}_{f}(E)+\sum_{j \in \check{I}} k_{j} \operatorname{ord}_{f}\left(\check{E}_{j}\right)= \\
\sum_{j \in I-\check{I}} k \operatorname{ord}_{f}\left(E_{j}\right)+\sum_{j \in I \cap \check{I}}\left(k+k_{j}\right) \operatorname{ord}_{f}\left(E_{j}\right)+\sum_{j \in \check{I}-I} k_{j} \operatorname{ord}_{f}\left(E_{j}\right) .
\end{array}
$$

Equations (2.3) and (2.4) tell us that $m d_{m}(\check{\pi}, f)=m d_{m}(\pi, f)$.

Lemma 2.6. The minimal multiplicity $m$ discrepancy does not depend on the choice of log resolution $\pi: Y \rightarrow \mathbb{C}^{n+1}$ of $\left(\mathbb{C}^{n+1}, f^{-1}(0)\right)$. 
Proof. Let $\pi: Y \rightarrow \mathbb{C}^{n+1}$ and $\check{\pi}: \check{Y} \rightarrow \mathbb{C}^{n+1}$ be two such resolutions. Lemma 2.5 tells us that blowing up along strata does not change the minimal multiplicity $m$ discrepancy. Hence by Lemma 2.4, we can assume that $\pi$ and $\check{\pi}$ are multiplicity $m$ separating resolutions.

Since $\pi$ and $\check{\pi}$ are birational morphisms, we have that there is a birational morphism $\Phi: Y \rightarrow \check{Y}$ so that $\pi=\check{\pi} \circ \Phi$. Let $\left(E_{j}\right)_{j \in S}$ be the resolution divisors of $\pi$ and $\left(\check{E}_{j}\right)_{j \in \check{S}}$ the resolution divisors of $\check{\pi}$. Suppose that $\operatorname{ord}_{f}\left(E_{j}\right)$ divides $m$ for some $j \in S-\star_{S}$. Let $\check{I} \subset \check{S}$ be the largest subset satisfying $\Phi\left(E_{j}\right) \subset \check{E}_{\check{I}}$.

Since $\Phi$ is well defined outside a subvariety of codimension $\geq 2$, we have a point $p \in E_{j}$ and holomorphic charts $y_{1}, \cdots, y_{n}$ in $Y$ and $x_{1}, \cdots, x_{n}$ in $\check{Y}$ centered at $p$ and $\Phi(p)$ respectively where $\Phi$ is well defined. We will also assume that $E_{j}=\left\{y_{1}=0\right\}$ and $\check{E}_{k}=\left\{x_{\alpha(k)}=0\right\}$ for all $k \in \check{I}$ and for some $\alpha: \check{I} \longrightarrow\{1, \cdots, n\}$. Let $J$ be the Jacobian of $\pi\left(y_{1}, \cdots, y_{n}\right), \breve{J}$ the Jacobian of $\check{\pi}\left(x_{1}, \cdots, x_{n}\right)$ and $J_{\Phi}$ the Jacobian of $\Phi\left(y_{1}, \cdots, y_{n}\right)$. Then

$$
\operatorname{ord}_{f}\left(E_{j}\right)=\sum_{k \in \check{I}} \operatorname{ord}_{x_{\alpha(k)} \circ \Phi}\left(E_{k}\right) \operatorname{ord}_{f}\left(\check{E}_{k}\right)
$$

and

$$
\operatorname{ord}_{J}\left(E_{j}\right)=\operatorname{ord}_{J_{\Phi}}\left(E_{j}\right)+\sum_{k \in \check{I}} \operatorname{ord}_{x_{\alpha(k)} \circ \Phi}\left(E_{k}\right) \operatorname{ord}_{\check{J}}\left(\check{E}_{k}\right) .
$$

By (2.5) combined with the fact that $\check{\pi}$ is a multiplicity $m$ separating resolution, we have that $\check{I}=\{k\}$ for some $k \in \check{S}-\star_{\check{S}}$. Hence $\operatorname{ord}_{f}\left(E_{j}\right)=\kappa_{\operatorname{ord}}\left(\check{E}_{k}\right)$ and $a\left(E_{j}\right) \geq \kappa a\left(\check{E}_{k}\right)$ where $\kappa=\operatorname{ord}_{x_{\alpha(k)} \circ \Phi}\left(E_{j}\right)$. Therefore by Lemma 2.3. $\operatorname{md}_{m}(\pi, f) \geq \operatorname{md}_{m}(\check{\pi}, f)$. Similarly $\operatorname{md}_{m}(\check{\pi}, f) \geq \operatorname{md}_{m}(\pi, f)$ and hence $\operatorname{md}_{m}(\pi, f)=\operatorname{md}_{m}(\check{\pi}, f)$.

\section{Liouville domains, Symplectomorphisms And Open Books}

In this section we give basic definitions of Liouville domains and graded symplectomorphisms and open books. We will also explain the correspondence between open book decompositions and graded symplectomorphisms of Liouville domains. All of the material here is contained in Gir02 with the exception of gradings which is contained in [Sei00]. For more details on open book decompositions see [Dï4].

Definition 3.1. An exact symplectic manifold is a pair $\left(M, \theta_{M}\right)$ where $M$ is a manifold and $\theta_{M}$ is a 1 -form so that $\omega_{M} \equiv d \theta_{M}$ is symplectic. A Liouville domain is an exact symplectic manifold $\left(M, \theta_{M}\right)$ where $M$ is a compact manifold with boundary and the $\omega_{M}$-dual $X_{\theta_{M}}$ of $\theta_{M}$ points outwards along $\partial M$. The 1 -form $\theta_{M}$ is called the Liouville form. The contact boundary of $M$ is the pair $\left(\partial M, \alpha_{M}\right)$ where $\left.\alpha_{M} \equiv \theta_{M}\right|_{\partial M}$. Here $\alpha_{M}$ is a contact form. Since $X_{\theta_{M}}$ points outwards along $\partial M$, we get that the backwards flow

$$
\left(\phi_{t}: M \hookrightarrow M\right)_{t \in(-\infty, 0]}
$$

of $X_{\theta_{M}}$ exists for all time $t$. By considering the smooth embeddings $\left.\phi_{\ln \left(r_{M}\right)}\right|_{\partial M}$ where $r_{M} \in$ $(0,1]$, we can construct a standard collar neighborhood $(0,1] \times \partial M \subset M$ of $\partial M$ where

$$
\left.\theta_{M}\right|_{(0,1] \times \partial M}=r_{M} \alpha_{M} .
$$

Here $r_{M}$ is the coordinate given by the natural projection $r_{M}:(0,1] \times \partial M \rightarrow(0,1]$ and is called the cylindrical coordinate on $M$.

An exact symplectomorphism $\phi: M \longrightarrow M$ is a diffeomorphism so that $\phi^{*} \theta_{M}=\theta_{M}+d F_{\phi}$ for some smooth function $F_{\phi}: M \rightarrow \mathbb{R}$. Technically we want to think of this as a pair $\left(\phi, F_{\phi}\right)$ 
but we will suppress $F_{\phi}$ from the notation and just write $\phi$. The support of such an exact symplectomorphism is the region

$$
\left\{x \in M: \phi(x) \neq x \quad \text { or } \quad d F_{\phi}(x) \neq 0\right\} .
$$

We now need to define graded symplectomorphisms as in [Sei00. This is needed so that we can define their Floer cohomology groups in the next section.

Definition 3.2. We define $\left(\mathbb{R}^{2 n}, \Omega_{s t d}\right)$ to be the standard symplectic vector space. Let $S p(2 n)$ be the space of linear symplectomorphisms of $\left(\mathbb{R}^{2 n}, \Omega_{s t d}\right)$ and $\widetilde{S p}(2 n)$ its universal cover. Let $\pi: E \longrightarrow V$ be a symplectic vector bundle with symplectic form $\Omega_{E}$ whose fibers have dimension $2 n$. Sometimes we will write $\left(E, \Omega_{E}\right)$ or just $E$ for such a symplectic vector bundle when the context is clear. Define the symplectic frame bundle $\operatorname{Fr}(E)$ to be an $S p(2 n)$ bundle whose fiber at $x \in V$ is the space of linear symplectomorphisms from $\left(\mathbb{R}^{2 n}, \Omega_{s t d}\right)$ to $\left(\pi^{-1}(x),\left.\Omega_{E}\right|_{x}\right)$. A grading on a symplectic vector bundle $\pi: E \longrightarrow V$ is an $\widetilde{S p}(2 n)$ bundle $\widetilde{F r}(E) \longrightarrow V$ together with a choice of isomorphism of $\widetilde{S p}(2 n)$ bundles

$$
\iota: \widetilde{F r}(E) \times \widetilde{S p}(2 n) S P(2 n) \cong \operatorname{Fr}(E) .
$$

This is just a choice of reduction of the structure group of $E$ from $S p(2 n)$ to $\widetilde{S p}(2 n)$. A symplectic vector bundle with a choice of grading is called a graded symplectic vector bundle. Suppose that $\check{\pi}: \check{E} \longrightarrow \check{V}$ is a symplectic vector bundle and $\widetilde{\beta}: \check{E} \longrightarrow E$ is a bundle morphism covering a smooth map $\beta: \check{V} \longrightarrow V$ so that $\widetilde{\beta}$ restricted to each fiber is a linear symplectomorphism. Let

$$
\widehat{\beta}: \check{E} \longrightarrow \beta^{*}(E), \quad \widehat{\beta}(\check{e})=(\check{\pi}(\check{e}), \widetilde{\beta}(\check{e}))
$$

be the natural isomorphism between this bundle and the pullback bundle. Then $\check{E}$ has a natural grading

$$
\begin{aligned}
& \beta^{*}(\widetilde{F r}(E)) \longrightarrow \check{V} \\
& \check{\iota}: \beta^{*}(\widetilde{F r}(E)) \times_{\widetilde{S p}(2 n)} S p(2 n)=\beta^{*}\left(\widetilde{F r}(E) \times_{\widetilde{S p}(2 n)} S p(2 n)\right) \longrightarrow \operatorname{Fr}(\check{E}) \text {, } \\
& \check{\iota}(\check{v}, w) \equiv \widehat{\beta}^{-1}((\check{v}, \iota(w))), \forall \check{v} \in \check{V}, w \in \widetilde{F r}(E) \times_{\widetilde{S p}(2 n)} S p(2 n)
\end{aligned}
$$

called the grading on E pulled back by $\widetilde{\beta}$.

The simplest example of a grading is the natural grading on the trivial bundle $\check{V} \times \mathbb{C}^{k} \longrightarrow \check{V}$ given by

$$
\begin{gathered}
\widetilde{F r}\left(\check{V} \times \mathbb{C}^{k}\right) \equiv \check{V} \times \widetilde{S p}(2 n), \\
\iota: \widetilde{F r}\left(\check{V} \times \mathbb{C}^{k}\right) \times \widetilde{S p(2 n)} S p(2 n)=\check{V} \times(\widetilde{S p}(2 n) \times \widetilde{S p}(2 n) \\
S p(2 n))=\check{V} \times S p(2 n) \rightarrow \operatorname{Fr}\left(\check{V} \times \mathbb{C}^{k}\right) .
\end{gathered}
$$

We will call such a grading the trivial grading.

Remark 3.3. In this paper we are only interested in gradings up to isotopy and so we will sometimes regard isotopic gradings as the same grading. If we have a smooth connected family of symplectic vector bundles then if one of them has a grading then all of them have a natural choice of grading up to isotopy. For more information about this see Appendix A.

Definition 3.4. Suppose that

$$
\pi_{1}: E_{1} \longrightarrow V_{1}, \quad \pi_{2}: E_{2} \longrightarrow V_{2}
$$


are graded symplectic vector bundles and $F: E_{1} \longrightarrow E_{2}$ is a symplectic vector bundle isomorphism covering a diffeomorphism $V_{1} \longrightarrow V_{2}$. Then a grading on $F$ is an $\widetilde{S p}(2 n)$ bundle isomorphism

$$
\widetilde{F}: \widetilde{F r}\left(E_{1}\right) \longrightarrow \widetilde{F r}\left(E_{2}\right)
$$

covering the $S p(2 n)$ bundle morphism $\operatorname{Fr}\left(E_{1}\right) \rightarrow \operatorname{Fr}\left(E_{2}\right)$ induced by $F$.

Let $\left(X, \omega_{X}\right)$ be a $2 n$ dimensional symplectic manifold. A grading on $(X, \omega)$ is a choice of grading on the symplectic vector bundle $T X$. A symplectic manifold with a choice of grading is called a graded symplectic manifold

A grading of a symplectomorpism $\phi$ between two graded symplectic manifolds $\left(X_{1}, \omega_{X_{1}}\right)$, $\left(X_{2}, \omega_{X_{2}}\right)$ is a choice of grading for the symplectic bundle isomorphism $d \phi: T X_{1} \longrightarrow T X_{2}$. A graded symplectomorphism is a symplectomorphism with a choice of grading.

We also wish to have a notion of grading for contact manifolds.

Definition 3.5. Recall that a cooriented contact manifold $\left(C, \xi_{C}\right)$ is a manifold $C$ of dimension $2 n-1$ with a cooriented hyperplane distribution $\xi_{C}$ with the property that there is a 1 -form $\alpha_{C}$ whose kernal is $\xi_{C}$ respecting this coorientation and so that $\alpha_{C} \wedge\left(d \alpha_{C}\right)^{n-1}$ is a volume form on $C$. The 1-form $\alpha_{C}$ is called a contact form compatible with $\xi_{C}$. A coorientation preserving contactomorphism between two cooriented contact manifolds is a diffeomorphism preserving their respective hyperplane distributions and coorientations. A contact submanifold $B \subset C$ is a submanifold so that $\left(B, \xi_{B} \equiv \xi_{C} \cap T B\right)$ is a cooriented contact manifold with the induced coorientation from $\xi_{C}$.

A grading on a contact manifold $\left(C, \xi_{C}\right)$ consists of a choice of contact form $\alpha_{C}$ compatible with $\xi_{C}$ and a choice of grading on the symplectic vector bundle $\left(\xi_{C},\left.d \alpha_{C}\right|_{\xi_{C}}\right)$. A cooriented contact manifold with a choice of grading is called a graded contact manifold. Since the space of contact forms compatible with $\xi_{C}$ is contractible, we get an induced grading on $\left(\xi_{C},\left.d \alpha\right|_{\xi_{C}}\right)$ for any other contact form $\alpha$ compatible with $\xi_{C}$ which is unique up to isotopy. Hence from now on we will regard this as the same grading.

A grading of a coorientation preserving contactomorphism $\phi$ between two graded contact manifolds $\left(C_{1}, \xi_{1}\right),\left(C_{2}, \xi_{2}\right)$ consists of a grading on the symplectic vector bundle isomorpism $\left.d \phi\right|_{\xi_{C_{1}}}: \xi_{C_{1}} \longrightarrow \xi_{C_{2}}$ where the symplectic forms on $\xi_{C_{1}}$ and $\xi_{C_{2}}$ come from contact forms $\alpha_{C_{1}}$ and $\alpha_{C_{2}}$ compatible with $\xi_{C_{1}}$ and $\xi_{C_{2}}$ respectively satisfying $\alpha_{C_{1}}=\phi^{*} \alpha_{C_{2}}$ with induced gradings. A graded contactomorphism is a coorientation preserving contactomorphism contactomorphism with a choice of grading.

In this paper, we will only deal cooriented contact manifolds and coorientation preserving contactomorphisms. So from now on, a contact manifold is a cooriented contact manifold and a contactomorphism is a coorientation preserving contactomorphism.

Definition 3.6. Suppose that $\left(C, \xi_{C}\right)$ is a contact manifold and $B$ is a contact submanifold. The normal bundle of $B$ is a symplectic vector bundle

$$
\pi_{\mathcal{N}_{C} B}: \mathcal{N}_{C} B \equiv\left(\left.T C\right|_{B}\right) / T B \rightarrow B
$$

with symplectic form defined as follows: Choose a compatible contact form $\alpha_{C}$ on $\left(C, \xi_{C}\right)$ and define

$$
T^{\perp} B \equiv\left\{\left.v \in \xi_{C}\right|_{x}: x \in B, \quad d \alpha_{C}(v, w)=0,\left.\left.\quad \forall w \in \xi_{C}\right|_{x} \cap T B\right|_{x} \cdot\right\}
$$

Then $T^{\perp} B$ is a symplectic vector bundle with symplectic form $\left.d \alpha_{C}\right|_{T^{\perp} B}$. The symplectic structure on $\mathcal{N}_{C} B$ is the pushforward of the above symplectic form under the natural bundle isomorphism $T^{\perp} B \rightarrow \mathcal{N}_{C} B$. 
Since the space of compatible contact forms is contractible, we have that the choice of symplectic form on the $\mathcal{N}_{C} B$ is unique up to symplectic bundle isomorphism and any two choices of such isomorphism are homotopic. As a result, we will refer to this bundle as the normal bundle as we are only concerned with isomorphisms of such bundles up to homotopy.

Definition 3.7. A contact pair with normal bundle data $\left(B \subset C, \xi_{C}, \Phi_{B}\right)$ consists of a contact manifold $\left(C, \xi_{C}\right)$ where $B$ is a codimension 2 contact submanifold along with a symplectic trivialization

$$
\Phi_{B}: \mathcal{N}_{C} B \rightarrow B \times \mathbb{C}
$$

of its normal bundle. A contactomorphism between two such triples

$$
\left(B_{1} \subset C_{1}, \xi_{C_{1}}, \Phi_{B_{1}}\right), \quad\left(B_{2} \subset C_{2}, \xi_{C_{2}}, \Phi_{B_{2}}\right)
$$

is a contactomorphism $\Psi: C_{1} \rightarrow C_{2}$ sending $B_{1}$ to $B_{2}$ so that the composition

$$
\mathcal{N}_{C_{1}} B_{1} \stackrel{\left.d \Psi\right|_{B_{1}}}{\longrightarrow} \mathcal{N}_{C_{2}} B_{2} \stackrel{\Phi_{B_{2}}}{\longrightarrow} B_{2} \times \mathbb{C} \stackrel{\left(\left.\Psi\right|_{B_{1}}\right)^{-1} \times \mathrm{id}_{\mathbb{C}}}{\longrightarrow} B_{1} \times \mathbb{C}
$$

is homotopic through symplectic bundle trivializations to $\Phi_{B_{1}}$. If there exists such a contactomorphism between the pairs as in Equation (3.2) then we say that they are contactomorphic.

A contact pair with normal bundle data $\left(B \subset C, \xi_{C}, \Phi_{B}\right)$ is graded if there is a choice of grading on $C-B$. A graded contactomorphism between two graded contact pairs with normal bundle data as in Equation (3.2) consists of a contactomorphism $\Psi$ between these contact pairs and a choice of grading of the contactomorphism $\left.\Psi\right|_{C_{1}-B_{1}}: C_{1}-B_{1} \longrightarrow C_{2}-B_{2}$. If a graded contactomorphism exists between two graded contact pairs with normal bundle data then we say that they are graded contactomorhic.

The main example of a contact pair with normal bundle data comes from singularity theory.

Example 3.8. Fix $n>0$. Let $f: \mathbb{C}^{n+1} \longrightarrow \mathbb{C}$ be a holomorphic function with an isolated singularity at 0 and let $J_{0}: T \mathbb{C}^{n+1} \longrightarrow T \mathbb{C}^{n+1}$ be the standard complex structure on $\mathbb{C}^{n+1}$. Let $S_{\epsilon} \subset \mathbb{C}^{n+1}$ be the sphere of radius $\epsilon>0$ and let $\xi_{S_{\epsilon}}=T S_{\epsilon} \cap J_{0} T S_{\epsilon}$ be the standard contact structure on $S_{\epsilon}$. Define $L_{f} \equiv f^{-1}(0) \cap S_{\epsilon}$. Then a result by Varchenko in Var82] tells us that for all sufficiently small $\epsilon>0, L_{f} \subset S_{\epsilon}$ is a contact submanifold called the link of $f$ at 0 . Also $d f$ gives us an induced map $\frac{d f}{d f} \mathcal{N}_{S_{\epsilon}} L_{f} \longrightarrow \mathbb{C}$ and hence a trivialization

$$
\Phi_{f} \equiv\left(i d_{L_{f}}, \overline{d f}\right): \mathcal{N}_{S_{\epsilon}} L_{f} \longrightarrow L_{f} \times \mathbb{C} .
$$

The contact pair with normal bundle data $\left(L_{f} \subset S_{\epsilon}, \xi_{S_{\epsilon}}, \Phi_{f}\right)$ is called the contact pair associated to $f$.

We also need a grading on this contact pair. It turns out, by the discussion in Definition 7.7. that every contact manifold with trivial first and second homology group has a unique grading up to homotopy. This means that $\xi_{S_{\epsilon}}$ has a grading giving us an induced grading on the contact pair associated to $f$. We will call this the standard grading.

We will now define open book decompositions and also graded open book decompositions. Let $\mathbb{D} \subset \mathbb{C}$ be the unit disk with polar coordinates $(r, \vartheta)$.

Definition 3.9. An open book is a pair $(C, \pi)$ where

- $C$ is a smooth manifold,

- $\pi: C-B \longrightarrow \mathbb{R} / \mathbb{Z}$ is a smooth fibration where $B$ is a codimension 2 submanifold and

- there is a tubular neighborhood $B \times \mathbb{D} \subset C$ of $B=B \times\{0\}$ in $C$ so that $\pi$ satisfies:

$$
\left.\pi\right|_{B \times(\mathbb{D}-0)}: B \times(\mathbb{D}-0) \longrightarrow \mathbb{R} / \mathbb{Z}, \quad \pi(x,(r, \theta))=2 \pi \theta .
$$


The submanifold $B$ is called the binding of the open book and a page of the open book is the closure of a fiber of $\pi$ which is a submanifold with boundary equal to $B$.

We now want open books to be compatible with contact pairs.

Definition 3.10. A contact pair with normal bundle data $\left(B \subset C, \xi_{C}, \Phi_{B}\right)$ is supported by an open book $(C, \pi)$

(1) if there is a contact form $\alpha_{C}$ compatible with $\xi_{C}$ so that $\left.d \alpha_{C}\right|_{\pi^{-1}(t)}$ is a symplectic form for all $t \in \mathbb{R} / \mathbb{Z}$,

(2) the trivialization of $\mathcal{N}_{C} B$ induced by the choice of tubular neighborhood from Definition 3.9 is homotopic through orientation preserving bundle trivializations to $\Phi_{B}$ (this does not depend on the choice of such a tubular neighborhood).

We will write $\left(C, \xi_{C}, \pi\right)$ for a contact pair supported by an open book and we will call it a contact open book. Note that $B$ and $\Phi_{B}$ are not included in the notation as $B=C-\operatorname{Dom}(\pi)$ and $\Phi_{B}$ is determined by the open book due to the fact that the space of orientation preserving trivializations of $\mathcal{N}_{C} B$ is weakly homotopic to the space of symplectic trivializations of $\mathcal{N}_{C} B$. The contact pair $\left(B \subset C, \xi_{C}, \Phi_{B}\right)$ is called the contact pair associated to $\left(C, \xi_{C}, \pi\right)$. If the contact pair associated to $\left(C, \xi_{C}, \pi\right)$ is graded then we call this a graded contact open book.

An isotopy between two contact open books $\left(C_{1}, \xi_{C_{1}}, \pi_{1}\right),\left(C_{2}, \xi_{C_{2}}, \pi_{2}\right)$ is a contactomorphism $\Phi: C_{1} \longrightarrow C_{2}$ between the respective contact pairs with normal bundle data together with a smooth family of maps $\left(\check{\pi}_{t}: \operatorname{Dom}\left(\pi_{1}\right) \longrightarrow \mathbb{R} / \mathbb{Z}\right)_{t \in[1,2]}$ joining $\pi_{1}$ and $\pi_{2} \circ \Phi$ so that $\left(C_{1}, \xi_{C_{1}}, \check{\pi}_{t}\right)$ is a contact open book for all $t \in[1,2]$. Such an isotopy is graded if we have a smooth family of graded contact open books and $\Phi$ is a graded contactomorphism.

The main example of a contact open book will come from singularity theory.

Example 3.11. Let $f: \mathbb{C}^{n+1} \longrightarrow \mathbb{C}, n>1$ be a holomorphic function with an isolated singularity at 0 and let $\left(L_{f} \subset S_{\epsilon}, \xi_{S_{\epsilon}}, \Phi_{f}\right)$ be the contact pair associated to $f$ as in Example 3.8. Let $\arg (f): \mathbb{C}^{n+1}-f^{-1}(0) \longrightarrow \mathbb{R} / 2 \pi \mathbb{Z}$ be the argument of $f$. Then by CNPP06, Proposition 3.4], we have that $\left(S_{\epsilon}, \xi_{S_{\epsilon}},\left.\frac{1}{2 \pi} \arg (f)\right|_{S_{\epsilon}}\right)$ is a contact open book for all $\epsilon>0$ small enough. This open book is supported by the contact pair associated to $f$ from Example 3.8 and it has a grading coming from the standard grading. This is a graded contact open book called the Milnor open book of $f$.

Definition 3.12. An abstract contact open book consists of a triple $\left(M, \theta_{M}, \phi\right)$ where $\left(M, \theta_{M}\right)$ is a Liouville domain and $\phi$ is an exact symplectomorphism with support in the interior of $M$. A graded abstract contact open book is an abstract contact open book $\left(M, \theta_{M}, \phi\right)$ with a choice of grading on $\left(M, d \theta_{M}\right)$ and a graded symplectomorphism $\phi$.

An isotopy between abstract contact open books $\left(M_{1}, \theta_{M_{1}}, \phi_{1}\right),\left(M_{2}, \theta_{M_{2}}, \phi_{2}\right)$ consists of a diffeomorphism $\Phi: M_{1} \longrightarrow M_{2}$, a smooth family of 1-forms $\left(\theta_{t}\right)_{t \in[0,1]}$ joining $\theta_{M_{1}}$ and $\Phi^{*} \theta_{M_{2}}$ and a smooth family of diffeomorphisms $\left(\check{\phi}_{t}\right)_{t \in[1,2]}$ joining $\phi_{1}$ and $\Phi^{-1} \circ \phi_{2} \circ \Phi$ so that $\left(M_{1}, \theta_{t}, \check{\phi}_{t}\right)$ are all abstract contact open books and the support of $\check{\phi}_{t}$ is contained inside a fixed compact subset of the interior of $M$. If both of our abstract contact open books are graded then such an isotopy is a graded isotopy if all the abstract contact open books $\left(M_{1}, \theta_{t}, \check{\phi}_{t}\right)$ are graded so that these gradings smoothly depend on $t \in[0,1]$ and the grading on $\left(M_{0}, \theta_{0}, \check{\phi}_{0}\right)$ coincides with the grading on $\left(M_{1}, \theta_{M_{1}}, \phi_{1}\right)$ and the grading on $\left(M_{1}, \theta_{1}, \check{\phi}_{1}\right)$ coincides with the grading on $\left(M_{2}, \theta_{M_{2}}, \phi_{2}\right)$ pulled back by $\Phi$.

From an abstract contact open book $\left(M, \theta_{M}, \phi\right)$, we wish to construct a contact open book. This construction is referred to as a generalized Thurston-Winkelnkemper construction in [Dï4, Section 2.2.1]. To do this we need the following definition. 
Definition 3.13. Let $\left(M, \theta_{M}, \phi\right)$ be an abstract contact open book. Let $F_{\phi}: M \longrightarrow \mathbb{R}$ be the smooth function with support in the interior of $M$ satisfying $\phi^{*} \theta_{M}=\theta_{M}+d F_{\phi}$. Let $\rho:[0,1] \longrightarrow[0,1]$ be a smooth function equal to 0 near 0 and 1 near 1 .

The mapping torus of $\left(M, \theta_{M}, \phi\right)$ is a smooth map

$$
\pi_{T_{\phi}}: T_{\phi} \longrightarrow \mathbb{R} / \mathbb{Z}, \quad T_{\phi} \equiv M \times[0,1] / \sim
$$

together with a contact form $\alpha_{T_{\phi}}$ on $T_{\phi}$ where

- $\sim$ identifies $(x, 1)$ with $(\phi(x), 0)$,

- $\alpha_{T_{\phi}} \equiv \theta_{M}+d\left(\rho(t) F_{\phi}\right)+C d t$ where $C>0$ is large enough to ensure that $\alpha_{T_{\phi}}$ is a contact form and

- $\pi_{T_{\phi}}(x, t) \equiv t \quad \forall(x, t) \in T_{\phi}=M \times[0,1] / \sim$.

For $\delta>0$ small enough, we have that the subset

$$
(1-\delta, 1] \times \partial M \subset(0,1] \times \partial M
$$

of the collar neighborhood of $\partial M$ is disjoint from the support of $\phi$. This means that there is a natural embedding:

$$
(1-\delta, 1] \times \partial M \times \mathbb{R} / \mathbb{Z} \subset T_{\phi}
$$

which we will call the standard collar neighborhood of $\partial T_{\phi}$. Note that $\alpha_{T_{\phi}}$ is equal to $r_{M} \alpha_{M}+$ $C d t$ in the standard collar neighborhood where $r_{M}$ (resp. $t$ ) is the natural projection map to $(1-\delta, 1](\operatorname{resp} . \mathbb{R} / \mathbb{Z})$ and $\alpha_{M}=\left.\theta_{M}\right|_{\partial M}$.

If $\left(M, \theta_{M}, \phi\right)$ is a graded abstract contact open book then we get a natural grading on $\left(T_{\phi}, \operatorname{ker}\left(\alpha_{T_{\phi}}\right)\right)$ as follows: Since the kernal of $d \alpha_{T_{\phi}}$ is transverse to the fibers of $T_{\phi}, \xi_{T_{\phi}} \equiv$ $\operatorname{ker}\left(\alpha_{T_{\phi}}\right)$ is isotopic through hyperplane distributions $H_{t}, t \in[0,1]$ to the vertical tangent space $T^{\mathrm{ver}} T_{\phi} \equiv \operatorname{ker}\left(D \pi_{\phi}\right)$ of $\pi_{T_{\phi}}$ with the property that $\left.d \alpha_{T_{\phi}}\right|_{H_{t}}$ is non-degenerate for all $t \in[0,1]$. Therefore it is sufficient to construct a grading for the symplectic vector bundle $\left(T^{\mathrm{ver}} T_{\phi}, d \alpha_{T_{\phi}}\right)$. Consider the symplectic vector bundle $\left(p r^{*} T M, p r^{*}\left(d \theta_{M}\right)\right)$ where

$$
p r: M \times[0,1] \longrightarrow M
$$

is the natural projection map. We have that the symplectic vector bundle $\left(T^{\mathrm{ver}} T_{\phi}, d \alpha_{T_{\phi}}\right)$ is isomorphic to the symplectic vector bundle on $\left(p r^{*} T M / \sim, p r^{*}\left(d \theta_{M}\right)\right)$ where $\sim$ identifies $\left.\operatorname{pr}^{*} T M\right|_{M \times\{1\}}$ with $\left.\operatorname{pr}^{*} T M\right|_{M \times\{0\}}$ using the map

$$
D \phi: T M=\left.p r^{*} T M\right|_{M \times\{1\}} \longrightarrow T M=\left.p r^{*} T M\right|_{M \times\{0\}} .
$$

Since $\left(T M, d \theta_{M}\right)$ has a natural grading, we get that $\left(p r^{*} T M, p r^{*}\left(d \theta_{M}\right)\right)$ has an induced grading

$$
\widetilde{F r}\left(p r^{*} T M\right) \times \widetilde{S p(2 n)} S p(2 n) \cong F r\left(p r^{*} T M\right)
$$

pulled back via $p r$. The map $D \phi$ gives us an induced map

$$
\left.\left.\operatorname{Fr}\left(p r^{*} T M\right)\right|_{M \times\{1\}} \longrightarrow F r\left(p r^{*} T M\right)\right|_{M \times\{0\}}
$$

and since $\phi$ is a graded symplectomorphism, the map above lifts to a map:

$$
\left.\left.\widetilde{F r}\left(p r^{*} T M\right)\right|_{M \times\{1\}} \longrightarrow \widetilde{F r}\left(p r^{*} T M\right)\right|_{M \times\{0\}} .
$$

Hence by using the above gluing map, we get a grading on the quotient $\left(p r^{*} T M / \sim, p r^{*}\left(d \theta_{M}\right)\right.$ ) and therefore a grading on $\left(T^{\mathrm{ver}} T_{\phi}, d \alpha_{T_{\phi}}\right)$. In turn this gives us a grading on the contact manifold $\left(T_{\phi}, \operatorname{ker}\left(\alpha_{T_{\phi}}\right)\right)$. We will call this the induced grading on $T_{\phi}$.

We will now construct a contact open book from an abstract contact open book. Let $\mathbb{D}(\rho) \subset \mathbb{C}$ be the closed disk of radius $\rho>0$ with polar coordinates $(r, \vartheta)$. 
Definition 3.14. Let $\left(M, \theta_{M}, \phi\right)$ be an abstract contact open book decomposition and let

$$
\pi_{T_{\phi}}: T_{\phi} \longrightarrow \mathbb{R} / \mathbb{Z}
$$

be the associated mapping torus with contact form $\alpha_{\phi}$ and standard collar neighborhood

$$
(1-\delta, 1] \times \partial M \times \mathbb{R} / \mathbb{Z} \subset T_{\phi}
$$

as in Definition 3.13. Define $C_{\phi} \equiv(\partial M \times \mathbb{D}(\delta)) \sqcup T_{\phi} / \sim$ where $\sim$ identifies $(x,(r, \vartheta)) \in$ $\partial M \times \mathbb{D}(\delta)$ with

$$
\left(1-r, x, \frac{\vartheta}{2 \pi}\right) \in(1-\delta, 1] \times \partial M \times \mathbb{R} / \mathbb{Z} \subset T_{\phi}
$$

We define $B_{\phi} \equiv \partial M \times\{0\} \subset \partial M \times \mathbb{D}(\delta) \subset C_{\phi}$ and

$$
\pi_{C_{\phi}}: C_{\phi}-B_{\phi}=T_{\phi}-\partial T_{\phi} \longrightarrow \mathbb{R} / \mathbb{Z}, \quad \pi_{C_{\phi}}=\left.\pi_{T_{\phi}}\right|_{T_{\phi}}-\partial T_{\phi} .
$$

Let

$$
h_{1}, h_{2}:[0, \delta) \longrightarrow \mathbb{R}
$$

be a pair of smooth functions so that

- $h_{1}^{\prime}(r)<0, h_{2}^{\prime}(r) \geq 0$ for all $r>0$,

- $h_{1}(r)=1-r^{2}$ and $h_{2}(r)=\frac{1}{2} r^{2}$ for small $r$ and

- $h_{1}(r)=1-r$ and $h_{2}(r)=C / 2 \pi$ for $r \in\left[\frac{\delta}{2}, \delta\right)$.

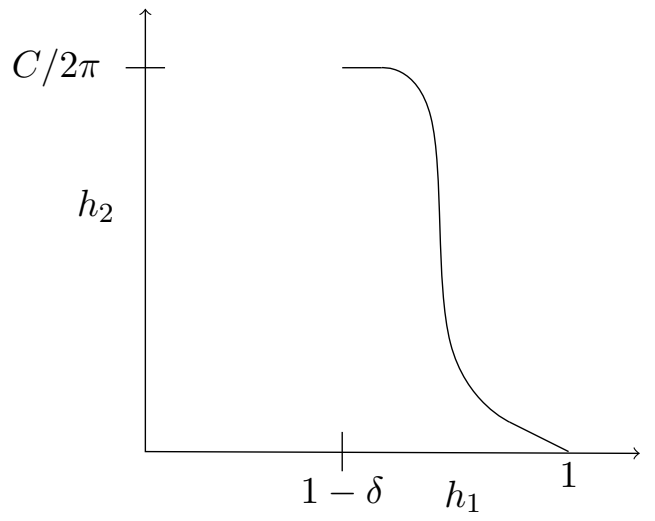

The above conditions ensure that

$$
\alpha_{C_{\phi}} \equiv\left\{\begin{array}{cl}
h_{1}(r) \alpha_{M}+h_{2}(r) d \vartheta & \text { inside } \partial M \times \mathbb{D}(\delta / 2) \\
\alpha_{T_{\phi}} & \text { inside } T_{\phi}-\partial M \times \mathbb{D}(\delta / 2) .
\end{array}\right.
$$

is a contact form whose restriction to $B_{\phi}$ is also a contact form and that the restriction of $d \alpha_{C_{\phi}}$ to $\pi^{-1}(t)$ is symplectic for all $t \in \mathbb{R} / \mathbb{Z}$. Define $\xi_{C_{\phi}} \equiv \operatorname{ker}\left(\alpha_{C_{\phi}}\right)$. The tubular neighborhood $\partial M \times \mathbb{D}(\delta / 2)$ of $B_{\phi}$ gives us a trivialization $\Phi_{B_{\phi}}$ of its normal bundle and hence we get a contact pair with normal bundle data $\left(B_{\phi} \subset C_{\phi}, \xi_{C_{\phi}}, \Phi_{B_{\phi}}\right)$ which we will called the contact pair associated to $\left(M, \theta_{M}, \phi\right)$. This contact pair with normal bundle data is supported by the open book $\left(C_{\phi}, \pi_{C_{\phi}}\right)$. Hence

$$
O B D\left(M, \theta_{M}, \phi\right) \equiv\left(C_{\phi}, \xi_{C_{\phi}}, \pi_{C_{\phi}}\right)
$$

is a contact open book which we call the open book associated to the abstract contact open book $\left(M, \theta_{M}, \phi\right)$.

Now suppose that $\left(M, \theta_{M}, \phi\right)$ is a graded abstract contact open book. Then since the contact manifold $\left(C_{\phi}-B_{\phi},\left.\xi_{C_{\phi}}\right|_{C_{\phi}-B_{\phi}}\right)$ is isotopic through contact manifolds to $T_{\phi}$, we get 
that the induced grading on $T_{\phi}$ gives us a grading on $\left(C_{\phi}-B_{\phi},\left.\xi_{C_{\phi}}\right|_{C_{\phi}-B_{\phi}}\right)$. Hence we have a relative grading on $\left(B_{\phi} \subset C_{\phi}, \xi_{C_{\phi}}, \Phi_{\phi}\right)$ which we will call the induced grading.

It is fairly straightforward to show that if two abstract contact open books are (graded) isotopic then their respective contact open book decompositions are (graded) isotopic. Hence we get a map

$$
O B D:\left\{\begin{array}{c}
\text { (graded) abstract contact } \\
\text { open books }
\end{array}\right\} \text { isotopy } \longrightarrow\{\text { (graded) open books }\} \text { /isotopy. }
$$

The Theorem below is a result of Giroux in Gir02.

Theorem 3.15. The above map $O B D$ is an bijection.

A detailed construction of the inverse of $O B D$ is contained in the proof of $\mathrm{D} \ddot{1} 4$, Theorem 3.1.22]. As a result of this Theorem, we have the following definition:

Definition 3.16. The monodromy map of a (graded) contact open book $\left(C, \xi_{C}, \pi\right)$ is defined to be the (graded) contactomorphism $\phi: M \longrightarrow M$ where $\left(M, \theta_{M}, \phi\right)$ is the abstract contact open book $O B D^{-1}\left(\left(C, \xi_{C}, \pi\right)\right)$.

Technically this monodromy map is only defined up to isotopy, and so the monodromy map is really just a choice of representative in this isotopy class.

\section{Fixed Point Floer Cohomology Definition}

Definition 4.1. Let $\left(M, \theta_{M}\right)$ be a Liouville domain. An almost complex structure $J$ on $M$ is cylindrical near $\partial M$ if it is compatible with the symplectic form $d \theta_{M}$ (I.e. $d \theta_{M}(\cdot, J(\cdot))$ is a Riemannian metric) and if $d r_{M} \circ J=-\alpha_{M}$ near $\partial M$ inside the standard collar neighborhood $(0,1] \times \partial M$.

An exact symplectomorphism $\phi: M \rightarrow M$ is non-degenerate if for every fixed point $p$ of $\phi$ the linearization of $\phi$ at $p$ as no eigenvalue equal to 1. It has small positive slope if it is equal to the time 1 Hamiltonian flow of $\delta r_{M}$ near $\partial M$ where $\delta>0$ is smaller than the period of the smallest periodic Reeb orbit of $\alpha_{M}$ (this means that it corresponds to the time $\delta$ Reeb flow near $\partial M$ ). If $\phi$ is an exact symplectomorphism, then a small positive slope perturbation $\check{\phi}$ of $\phi$ is an exact symplectomorphism $\check{\phi}$ equal to the composition of $\phi$ with a $C^{\infty}$ small Hamiltonian symplectomorphism of small positive slope. The action of a fixed point $p$ is $-F_{\phi}(p)$ where $F_{\phi}$ is a function satisfying $\phi^{*} \theta_{M}=\theta_{M}+d F_{\phi}$. The action depends on a choice of $F_{\phi}$ which has to be fixed when $\phi$ is defined although usually $F_{\phi}$ is chosen so that it is zero near $\partial M$ (if possible). All of the symplectomorphisms coming from isolated hypersurface singularities will have such a unique $F_{\phi}$. An isolated family of fixed points is a path connected compact subset $B \subset M$ consisting of fixed points of $\phi$ of the same action and for which there is a neighborhood $N \supset B$ where $N \backslash B$ has no fixed points. Such an isolated family of fixed points is called a codimension 0 family of fixed points if in addition there is an autonomous Hamiltonian $H: N \rightarrow(-\infty, 0]$ so that $H^{-1}(0)=B$ is a connected codimension 0 submanifold with boundary and corners, the time $t$ flow of $X_{H}$ is well defined for all $t \in \mathbb{R}$ and $\left.\phi\right|_{N}: N \rightarrow N$ is equal to the time 1 flow of $H$. The action of an isolated family of fixed points $B \subset M$ is the action of any point $p \in B$.

Before we define Floer cohomology, we need some definitions so that we can give it a grading. For any path of symplectic matrices $\left(A_{t}\right)_{t \in[a, b]}$ we can assign an index $C Z\left(A_{t}\right)$ called its Conley-Zehnder index. The Conley-Zehnder index of certain paths of symplectic matrices $A_{t}$ was originally defined in CZ84. It was defined for a general path of symplectic 
matrices in RS93 and also in Gut13. We will not define it here as we will only use the following properties (see [Gut13, Proposition 8]):

(CZ1) $C Z\left(\left(e^{i t}\right)_{t \in[0,2 \pi]}\right)=2$.

(CZ2) $(-1)^{n-C Z\left(\left(A_{t}\right)_{t \in[0,1]}\right)}=\operatorname{sign} \operatorname{det}_{\mathbb{R}}\left(\mathrm{id}-A_{1}\right)$ for any path of symplectic matricies $\left(A_{t}\right)_{t \in[0,1]}$.

(CZ3) $C Z\left(A_{t} \oplus B_{t}\right)=C Z\left(A_{t}\right)+C Z\left(B_{t}\right)$.

(CZ4) The Conley-Zehnder index of the catenation of two paths is the sum of their ConleyZehnder indices.

(CZ5) If $A_{t}$ and $B_{t}$ are two paths of symplectic matrices which are homotopic relative to their endpoints then they have the same Conley-Zehnder index. Also such an index only depends on the path up to orientation preserving reparameterization.

Definition 4.2. Let $\left(M, \theta_{M}, \phi\right)$ be a graded abstract contact open book. The Conley-Zehnder index $C Z(p)$ of a fixed point $p$ of the graded symplectomorphism $\phi$ is defined as follows: Since $\left(T M, d \theta_{M}\right)$ is a graded symplectic vector bundle, we have an associated $\widetilde{S p}(2 n)$ bundle

$$
\widetilde{F r}(T M) \longrightarrow M
$$

together with a choice of isomorphism of $\widetilde{S p}(2 n)$ bundles

$$
\iota: \widetilde{F r}(T M) \times_{\widetilde{S p}(2 n)} S p(2 n) \cong \operatorname{Fr}(T M) .
$$

Now choose an identification of $\widetilde{S p}(2 n)$ torsors

$$
\widetilde{S p}(2 n)=\left.\widetilde{F r}(T M)\right|_{p} .
$$

The symplectomorphism $\phi$ has a choice of grading giving us a natural map:

$$
\widetilde{\phi}:\left.\left.\widetilde{F r}(T M)\right|_{p} \longrightarrow \widetilde{F r}(T M)\right|_{p}
$$

and hence by Equation (4.1), a map

$$
\widetilde{\phi}: \widetilde{S p}(2 n) \longrightarrow \widetilde{S p}(2 n) .
$$

Since $\widetilde{S p}(2 n)$ is the universal cover of $S p(2 n)$, its elements correspond to paths of symplectic matrices starting from the identity up to homotopy fixing their endpoints and so let $\beta$ be the path corresponding to $\widetilde{\phi}(i d) \in \widetilde{S p}(2 n)$. We define $C Z(\phi, p) \equiv C Z(\beta)$.

If we have an isolated family of fixed points $B \subset X$, then we define its Conley-Zehnder index $C Z(\phi, B)$ to be the Conley-Zehnder index of some $b \in B$. Since $B$ is path connected, this does not depend on the choice of $b \in B$ by property (CZ5) above.

In Appendix A, we also have a different way of computing the Conley-Zehnder index by looking at the mapping torus $T_{\phi}$ of $\phi$. This will be useful in the proof of Theorem 5.41.

Definition 4.3. Let $\left(M, \theta_{M}, \phi\right)$ be an abstract contact open book. Let $\left(J_{t}\right)_{t \in[0,1]}$ be a smooth family of almost complex structures with the property that $\phi^{*} J_{0}=J_{1}$. A Floer trajectory of $\left.\left(\phi, J_{t}\right)_{t \in[0,1]}\right)$ joining $p_{-}, p_{+} \in M$ is a smooth map $u: \mathbb{R} \times[0,1] \rightarrow M$ so that $\partial_{s} u+J_{t} \partial_{t} u=0$ where $(s, t)$ parameterizes $\mathbb{R} \times \mathbb{R} / \mathbb{Z}, u(s, 0)=\phi(u(s, 1))$ and so that $\lim _{s \rightarrow \pm \infty} u(s, t)=p_{ \pm}$ for all $t \in[0,1]$. We write $\mathcal{M}\left(\phi, J_{t}, p_{-}, p_{+}\right)$for the set of such Floer trajectories and define $\overline{\mathcal{M}}\left(\phi, J_{t}, p_{-}, p_{+}\right) \equiv \mathcal{M}\left(\phi, J_{t}, p_{-}, p_{+}\right) / \mathbb{R}$, where $\mathbb{R}$ acts by translation in the $s$ coordinate.

Let $\left(M, \theta_{M}, \phi\right)$ be a graded abstract contact open book. We will now give a sketch of the definition of the Floer cohomology group $H F^{*}(\phi,+)$ (see [Sei01]). Let $\check{\phi}$ be a small positive slope perturbation of $\phi$. This can be done so that $\check{\phi}$ is $C^{\infty}$ close to $\phi$ and so that the fixed 
points of $\check{\phi}$ are non-degenerate (see [SZ92, Theorem 9.1] in the case where $\check{\phi}$ is Hamiltonian. The general case is similar [DS94, Page 586]). We can also ensure that $\check{\phi}$ is a graded symplectomorphism due to the fact that it is isotopic to $\phi$ through symplectomorphisms.

We now choose a $C^{\infty}$ generic family of cylindrical almost complex structures $\left(J_{t}\right)_{t \in[0,1]}$ satisfying $\phi^{*} J_{0}=J_{1}$. The genericity property then tells us that $\overline{\mathcal{N}}\left(\check{\phi}, J_{t}, p_{-}, p_{+}\right)$is a compact oriented manifold of dimension 0 for all fixed points $p_{-}, p_{+}$of $\phi$ satisfying $C Z\left(p_{-}\right)-C Z\left(p_{+}\right)=$ 1 ([DS94, Theorem 3.2]). We define $\#^{ \pm} \overline{\mathcal{M}}\left(\check{\phi}, J_{t}, p_{-}, p_{+}\right)$to be the signed count of elements of $\overline{\mathcal{M}}\left(\check{\phi}, J_{t}, p_{-}, p_{+}\right)$. Let $C F^{*}(\check{\phi})$ be the free abelian group generated by fixed points of $\phi$ and

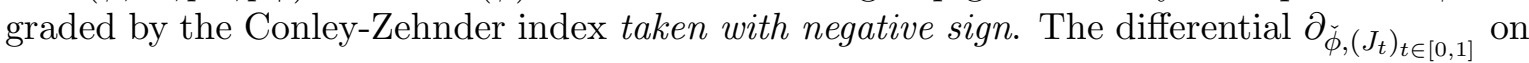

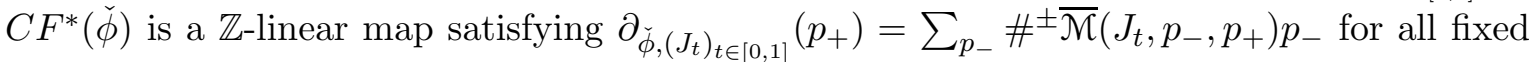
points $p_{+}$of $\check{\phi}$ where the sum is over all fixed points $p_{-}$satisfying $\left(-C Z\left(p_{-}\right)\right)-\left(-C Z\left(p_{+}\right)\right)=$ 1. Because $\left(J_{t}\right)_{t \in[0,1]}$ is $C^{\infty}$ generic, one can show that $\partial_{\check{\phi},\left(J_{t}\right)_{t \in[0,1]}^{2}}^{2}=0$ ([DS94, Theorem 3.3(1)]) and we define the resulting homology group to be $H F^{*}\left(\check{\phi},\left(J_{t}\right)_{t \in[0,1]}\right)$. We define $H F^{*}(\phi,+) \equiv H F^{*}\left(\check{\phi},\left(J_{t}\right)_{t \in[0,1]}\right)$. This does not depend on the choice of perturbation $\check{\phi}$ or on the choice of almost complex structure $\left(J_{t}\right)_{t \in[0,1]}$ ([DS94, Theorem 3.3(2)]). Our conventions then tell us that if $\phi: M \longrightarrow M$ is the identity map with the trivial grading and $\operatorname{dim}(M)=n$ then $H F^{k}(\phi,+)=H^{n+k}(M ; \mathbb{Z})$.

We will only use the following properties of these Floer cohomology groups:

(HF1) For a graded abstract contact open book $\left(M, \theta_{M}, \phi\right)$, the Lefschetz number $\Lambda(\phi)$ of $\phi$ is equal to the Euler characteristic of $H F^{*}(\phi,+)$ multiplied by $(-1)^{n}$ (follows from $(\mathrm{CZ2})$.

(HF2) Suppose that $\left(M_{1}, \theta_{M_{1}}, \phi_{1}\right),\left(M_{2}, \theta_{M_{2}}, \phi_{2}\right)$ are graded abstract contact open books so that the graded contact pairs associated to them are graded contactomorphic. Then $H F^{*}\left(\phi_{0},+\right)=H F^{*}\left(\phi_{1},+\right)$ (See the Appendix A).

(HF3) Let $\left(M, \theta_{M}, \phi\right)$ be a graded abstract contact open book where $\operatorname{dim}(M)=2 n$. Suppose that the set of fixed points of a small positive slope perturbation $\check{\phi}$ of $\phi$ is a disjoint union of codimension 0 families of fixed points $B_{1}, \cdots, B_{l}$ and let $\iota:\{1, \cdots, l\} \longrightarrow \mathbb{N}$ be a function where

- $\iota(i)=\iota(j)$ if and only if the action of $B_{i}$ equals the action of $B_{j}$ and

- $\iota(i)<\iota(j)$ if the action of $B_{i}$ is less than the action of $B_{j}$.

Then there is a cohomological spectral sequence converging to $H F^{*}(\phi,+)$ whose $E_{1}$ page is equal to

$$
E_{1}^{p, q}=\bigoplus_{\{i \in\{1, \cdots, l\}: \iota(i)=p\}} H_{n-(p+q)-C Z\left(\phi, B_{j}\right)}\left(B_{p} ; \mathbb{Z}\right)
$$

(see Appendix C).

\section{Constructing a Well Behaved Contact Open Book.}

The aim of this section is to modify the Milnor monodromy map so that the set of fixed points is a union of codimension 0 families of fixed points so that we can apply (HF3) above. We will do this by constructing such a nice symplectomorphism whose mapping torus is isotopic to the mapping torus of the Milnor monodromy map.

5.1. Some Preliminary Definitions. The aim of this section is to construct a symplectic form on the resolution which behaves well with respect to the resolution divisors. To do this 
we need a purely symplectic notion of smooth normal crossing divisor. We will introduce some notation from [MTZ14a].

If $V \subset X$ is a submanifold of a manifold $X$ then we will use the notation

$$
\pi_{\mathcal{N}_{X} V}: \mathcal{N}_{X} V \equiv \frac{\left.T X\right|_{V}}{T V} \rightarrow V
$$

for the normal bundle of $V$. If $\left(V_{i}\right)_{i \in S}$ is a finite collection of submanifolds of $X$ and $I \subset S$, let

$$
V_{I} \equiv \bigcap_{i \in I} V_{i}
$$

Also by convention we define $V_{\emptyset} \equiv X$. The collection $\left(V_{i}\right)_{i \in S}$ intersects transversally if for every subset $I \subset S$ and every $x \in V_{I}$,

$$
\operatorname{codim}_{T_{x} X}\left(\bigcap_{i \in I} T_{x} V_{i}\right)=\sum_{i \in I} \operatorname{codim}_{T_{x} X}\left(T_{x} V_{i}\right) .
$$

If $V \subset X$ is a submanifold and $X$ is oriented then an orientation on $V$ induces an orientation on $\mathcal{N}_{X} V$ and conversely an orientation on $\mathcal{N}_{X} V$ induces an orientation on $V$ using Equation (5.1) (if $V$ is odd dimensional this depends on a sign convention but we will not need this since the manifolds that we will be dealing with are even dimensional). If $X$ is oriented and $\left(V_{i}\right)_{i \in S}$ is a collection of oriented transversally intersecting submanifolds, then the submanifold $V_{I}$ has a natural orientation called the intersection orientation since $\mathcal{N}_{X} V_{I}=\bigoplus_{i \in I} \mathcal{N}_{X} V_{i}$ is oriented.

Definition 5.1. Suppose that $(X, \omega)$ is a symplectic manifold. Then $\left(V_{i}\right)_{i \in S}$ is called a symplectic crossings divisor or $S C$ divisor if $\left(V_{i}\right)_{i \in S}$ are transversally intersecting codimension 2 symplectic submanifolds so that $V_{I}$ is also symplectic and so that the symplectic orientation on $V_{I}$ agrees with its corresponding intersecting orientation for all $I \subset S$. We will also assume that $S$ is a finite set.

We now want to define SC divisors with particularly nice neighborhoods. We call

$$
\pi:(L, \rho, \nabla) \rightarrow V
$$

a Hermitian line bundle if $\pi: L \rightarrow V$ is a complex line bundle, $\rho$ is a Hermitian metric and $\nabla$ is a $\rho$-compatible Hermitian connection on $L$. We define $\rho^{\mathbb{R}}$ and $\rho^{i \mathbb{R}}$ to be the real and complex parts of the Hermitian metric $\rho$. We will also use the notation $\rho$ to denote square of the norm function on $L$. If we view $L$ as an oriented real vector bundle then we can recover the complex structure $\mathbf{i}_{\rho}$ from the metric $\rho^{\mathbb{R}}$ using the fact that for all $x \in V$ and $\left.W \in L\right|_{x}-0, \mathbf{i}_{\rho}(W)$ is the unique vector making $W, \mathbf{i}_{\rho}(W)$ into an oriented orthonormal basis of $\left.L\right|_{x}$. Hence we can define a Hermitian structure $(\rho, \nabla)$ on any oriented 2-dimensional real vector bundle $L \rightarrow V$ to be a pair $(\rho, \nabla)$ making $\left(L, \mathbf{i}_{\rho}\right)$ into a Hermitian line bundle.

For any such triple $(L, \rho, \nabla)$ we have an associated Hermitian connection 1-form $\alpha_{\rho, \nabla} \in$ $\Omega^{1}(L-V)$. This is the pullback of the associated principal $U(1)$-connection on the unit circle bundle of $(L, \rho)$ (see [BW58] or [MTZ14a, Appendix A]). If $\left(L_{i}, \rho_{i}, \nabla^{(i)}\right)_{i \in I}$ is a finite collection of Hermitian line bundles over a symplectic manifold $(V, \omega)$ and $p r_{I ; i}: \bigoplus_{i \in I} L_{i} \rightarrow L_{i}$ is the natural projection map then we define

$$
\omega_{\left(\rho_{i}, \nabla^{(i)}\right)_{i \in I}} \equiv \pi^{*} \omega+\frac{1}{2} \sum_{i \in I} p r_{I ; i}^{*} d\left(\rho \alpha_{\rho_{i}, \nabla^{(i)}}\right) .
$$

This is a symplectic form in some small neighborhood of the zero section. Given a 2dimensional symplectic vector bundle $L \rightarrow V$ with symplectic form $\Omega$, an $\Omega$-compatible 
Hermitian structure on $L$ is a Hermitian structure $(\rho, \nabla)$ where the complex structure $\mathbf{i}_{\rho}$ is compatible with $\Omega$.

Suppose that $\Psi: \check{V} \rightarrow V$ is a diffeomorphism and suppose $\pi:\left(L_{i}, \rho_{i}, \nabla^{(i)}\right)_{i \in I} \rightarrow V, \check{\pi}:$ $\left(\check{L}_{i}, \check{\rho}_{i}, \check{\nabla}^{(i)}\right)_{i \in I} \rightarrow \check{V}$ are two collections of Hermitian line bundles then a product Hermitian isomorphism is a vector bundle isomorphism

$$
\widetilde{\Psi}: \bigoplus_{i \in I} \check{L}_{i} \rightarrow \bigoplus_{i \in I} L_{i}
$$

covering $\Psi$ and respecting the direct sum decomposition so that the induced map $\widetilde{\Psi}$ : $\left(\check{L}_{i}, \check{\rho}_{i}, \check{\nabla}^{(i)}\right) \rightarrow\left(L_{i}, \rho_{i}, \nabla^{(i)}\right)$ is an isomorphism of Hermitian line bundles for all $i \in \check{I}$.

Definition 5.2. Let $V \subset X$ be a submanifold of a manifold $X$. A regularization is a diffeomorphism $\Psi: \check{\mathcal{N}} \rightarrow X$ from a neighborhod $\check{\mathcal{N}} \subset \mathcal{N}_{X} V$ of the zero section onto its image so that $\Psi(x)=x$ for all $x \in V$ and so that the map

$$
d_{x} \Psi:\left.\left.\mathcal{N}_{X} V\right|_{x} \longrightarrow \mathcal{N}_{X} V\right|_{x}, \quad d_{x} \Psi(v) \equiv Q\left(D \Psi\left(\left.\frac{d}{d t}(t v)\right|_{t=0}\right)\right)
$$

is the identity map where $Q:\left.T X\right|_{V} \longrightarrow \mathcal{N}_{X} V$ is the natural quotient map.

We also need a notion of regularization compatible with the symplectic form. Because of this we first need to talk about connections induced by closed 2-forms. Recall that an Ehresmann connection on a smooth submersion $\pi: E \longrightarrow B$ is a distribution $H \subset T E$ so that $\left.D \pi\right|_{H_{x}}: H_{x} \longrightarrow T_{\pi(x)} B$ is an isomorphism for all $x \in E$.

Definition 5.3. Let $\pi: E \longrightarrow B$ be a smooth submersion and let $\Omega$ be a 2 -form on $E$ whose restriction to each fiber is non-degenerate. Then the symplectic connection associated to $\Omega$ is an Ehresmann connection $H \subset T E$ where $H$ is the set of vectors which are $\Omega$-orthogonal to the fibers of $\pi$. In other words,

$$
H=\left\{V \in T_{x} E: x \in E, \quad \Omega(V, W)=0 \quad \forall W \in T_{x}^{\mathrm{ver}} E\right\}
$$

where $T_{x}^{\mathrm{ver}} E \equiv \operatorname{ker}(D \pi)$ is the vertical tangent bundle.

If $(X, \omega)$ is a symplectic manifold and $V \subset X$ is a symplectic submanifold then $\mathcal{N}_{X} V$ is a symplectic vector bundle on $V$. We write $\left.\omega\right|_{\mathcal{N}_{X} V}$ for the symplectic form on this vector bundle and $\left.\omega\right|_{L}$ for the restriction of $\left.\omega\right|_{\mathcal{N}_{X} V}$ to $L$ where $L$ is any subbundle $L \subset \mathcal{N}_{X} V$. The following definition differs in notation from [MTZ14b, Definition 2.8] but it defines the same object.

Definition 5.4. Let $(X, \omega)$ be a symplectic manifold, $V$ a symplectic submanifold and let

$$
\mathcal{N}_{X} V \equiv \bigoplus_{i \in I} L_{i}
$$

be a splitting into 2-dimsional subbundles so that $\left.\omega\right|_{L_{i}}$ is non-degenerate for all $i \in I$. A tuple $\left(\left(\rho_{i}\right)_{i \in I}, \Psi\right)$ is called an $\omega$-regularization for $V$ in $X$ if $\Psi$ is a regularization for $V$ in $X$ and $\rho_{i}$ is a map $\rho_{i}: \operatorname{Im}(\Psi) \longrightarrow[0, \infty)$ so that there is an $\left.\omega\right|_{L_{i}}$-compatible Hermitian structure $\left(\widetilde{\rho}_{i}, \nabla^{(i)}\right)$ on $L_{i}$ satisfying

- $\left.\widetilde{\rho}_{i}\right|_{\operatorname{Dom}(\Psi)}=\rho_{i} \circ \Psi$ for all $i \in I$ where $\widetilde{\rho}_{i}$ is (by abuse of notation) the pullback of $\widetilde{\rho}_{i}$ by the natural projection map $\oplus_{j \in I} L_{j} \longrightarrow L_{i}$,

- $\nabla^{(i)}$ restricted to $\operatorname{Dom}(\Psi) \cap L_{i}$ coincides with the symplectic connection associated to $\left.\omega\right|_{L_{i}}$ for all $i \in I$ and

$$
\Psi^{*} \omega=\omega_{\left(\widetilde{\rho}_{i}, \nabla^{(i)}\right)_{i \in I}} \mid \operatorname{Dom}(\Psi)
$$


for each $i \in I$.

The splitting (5.3) is called the associated splitting and the $\left.\omega\right|_{L_{i}}$-compatible Hermitian structure $\left(\widetilde{\rho}_{i}, \nabla^{(i)}\right)$ is called the associated Hermitian structure on $L_{i}$. This Hermitian structure is uniquely determined by $\rho_{i}$ and $\Psi$. Also since $\widetilde{\rho}_{i}$ gives us a complex structure on $L_{i}$ for each $i \in I$, we get a natural complex structure on $\mathcal{N}_{X} V$ which we will call the complex structure associated to the $\omega$-regularization $\left(\left(\rho_{i}\right)_{i \in I}, \Psi\right)$.

We wish to extend Definitions 5.2 and 5.4 to transverse collections of submanifolds and SC divisors respectively. To do this we need some preliminary notation. Let $X$ be a manifold and $\left(V_{i}\right)_{i \in S}$ transversely intersecting submanifolds. We have a canonical identification

$$
\mathcal{N}_{X} V_{I}=\pi_{I ; I^{\prime}}^{*}\left(\mathcal{N}_{V_{I^{\prime}}} V_{I}\right)
$$

for each $I^{\prime} \subset I$ where

$$
\pi_{I ; I^{\prime}}: \mathcal{N}_{V_{I-I^{\prime}}} V_{I} \rightarrow V_{I}
$$

is the natural projection map. Note that (5.5) is not an identification of bundles since the base of the left hand bundle is $V_{I}$ whereas the base of the right hand bundle is $\mathcal{N}_{V_{I-I^{\prime}}} V_{I}$.

Definition 5.5. Let $X$ be a manifold and $\left(V_{i}\right)_{i \in S}$ a transverse collection of submanifolds. A system of regularizations is a tuple $\left(\Psi_{I}\right)_{I \subset S}$, where $\Psi_{I}$ is a regularization for $V_{I}$ so that

$$
\Psi_{I}\left(\left.\mathcal{N}_{V_{I^{\prime}}}\right|_{V_{I}} \cap \operatorname{Dom}\left(\Psi_{I}\right)\right)=V_{I^{\prime}} \cap \operatorname{Im}\left(\Psi_{I}\right)
$$

for all $I^{\prime} \subset I \subset S$.

Define

$$
\iota: \pi_{I ; I^{\prime}}^{*}\left(\mathcal{N}_{V_{I^{\prime}}} V_{I}\right) \longleftrightarrow T \pi_{I ; I^{\prime}}^{*}\left(\mathcal{N}_{V_{I^{\prime}}} V_{I}\right) \stackrel{5.5}{=} T \mathcal{N}_{X} V_{I},\left.\quad \iota(x, v) \equiv \frac{d}{d t}(x, t v)\right|_{t=0}
$$

Using the inclusion map $\iota$ above, define

$$
\mathfrak{D} \Psi_{I ; I^{\prime}}:\left.\pi_{I ; I^{\prime}}^{*}\left(\mathcal{N}_{V_{I^{\prime}}} V_{I}\right)\right|_{\Psi_{I}^{-1}\left(V_{I^{\prime}}\right)} \longrightarrow \mathcal{N}_{X}\left(V_{I^{\prime}} \cap \operatorname{Im}\left(\Psi_{I}\right)\right), \quad \mathfrak{D} \Psi_{I ; I^{\prime}}(w) \equiv Q\left(D \Psi_{I}(\iota(w))\right)
$$

where $Q:\left.T X\right|_{V_{I^{\prime}} \cap \operatorname{Im}\left(\Psi_{I}\right)} \longrightarrow \mathcal{N}_{X}\left(V_{I^{\prime}} \cap \operatorname{Im}\left(\Psi_{I}\right)\right)$ is the natural projection map.

Using the equality (5.5), $\mathfrak{D} \Psi_{I ; I^{\prime}}$ identifies the normal bundle of $\mathcal{N}_{V_{I-I^{\prime}}} V_{I}$ inside $\mathcal{N}_{X} V_{I}$ near 0 with the normal bundle of $V_{I^{\prime}}$ near $V_{I}$ using the derivative of the regularization $\Psi_{I}$. The map $\mathfrak{D} \Psi_{I ; I^{\prime}}$ is also a bundle isomorphism covering the diffeomorphism

$$
\left.\Psi_{I}\right|_{\Psi_{I}^{-1}\left(V_{I^{\prime}}\right)}: \Psi_{I}^{-1}\left(V_{I^{\prime}}\right) \longrightarrow V_{I^{\prime}} \cap \operatorname{Im}\left(\Psi_{I}\right) .
$$

The definition below tells us that $\Psi_{I^{\prime}}$ and $\Psi_{I}$ should be equal under the identification (5.7).

Definition 5.6. Let $X$ be a manifold and $\left(V_{i}\right)_{i \in S}$ a transverse collection of submanifolds of $X$. Then a regularization for $\left(V_{i}\right)_{i \in S}$ is a system of regularizations $\left(\Psi_{I}\right)_{I \subset S}$ for $\left(V_{i}\right)_{i \in S}$ so that:

$$
\mathfrak{D} \Psi_{I ; I^{\prime}}\left(\operatorname{Dom}\left(\Psi_{I}\right)\right)=\left.\operatorname{Dom}\left(\Psi_{I^{\prime}}\right)\right|_{V_{I^{\prime}} \cap \operatorname{Im}\left(\Psi_{I}\right)}, \quad \Psi_{I}=\left.\Psi_{I^{\prime}} \circ \mathfrak{D} \Psi_{I ; I^{\prime}}\right|_{\operatorname{Dom}\left(\Psi_{I}\right)} .
$$

The following definition differs from [MTZ14b, Definition 2.11] for the same reasons that Definition 5.4differs from MTZ14b, Definition 2.8]. Apart from that, this definition is exactly the same. This structure also appears in [McL12a, Lemma 5.14] although the regularization maps have particular domains and it is defined in a slightly different way.

Definition 5.7. Let $(X, \omega)$ be a symplectic manifold and $\left(V_{i}\right)_{i \in S}$ an SC divisor. An $\omega$ regularization is a pair of tuples

$$
\left(\left(\rho_{i}\right)_{i \in S},\left(\Psi_{I}\right)_{I \subset S}\right)
$$

where 
FLOER COHOMOLOGY, MULTIPLICITY AND THE LOG CANONICAL THRESHOLD.

(1) $\left(\Psi_{I}\right)_{I \subset S}$ is a regularization for $\left(V_{i}\right)_{i \in S}$ as in Definition 5.6 and

$$
\rho_{i}: \bigcup_{i \in I \subset S} \operatorname{Im}\left(\Psi_{I}\right) \longrightarrow[0, \infty)
$$

is a smooth map,

(2) $\left(\left(\left.\rho_{i}\right|_{\operatorname{Im}\left(\Psi_{I}\right)}\right)_{i \in I}, \Psi_{I}\right)$ is an $\omega$-regularization for $V_{I}$ in $X$ for each $I \subset S$ as in Definition 5.4 and

(3) the maps $\mathfrak{D} \Psi_{I ; I^{\prime}}$ from (5.7) are product Hermitian isomorphisms for all $I^{\prime} \subset I \subset S$ with respect to the natural splittings

$$
\begin{gathered}
\left.\pi_{I ; I^{\prime}}^{*}\left(\mathcal{N}_{V_{I^{\prime}}} V_{I}\right)\right|_{\Psi_{I}^{-1}\left(V_{I^{\prime}}\right)}=\left.\bigoplus_{i \in I^{\prime}} \pi_{I ; I^{\prime}}^{*}\left(\left.\mathcal{N}_{X} V_{i}\right|_{V_{I}}\right)\right|_{\Psi_{I}^{-1}\left(V_{I^{\prime}}\right)}, \\
\mathcal{N}_{X}\left(V_{I^{\prime}} \cap \operatorname{Im}\left(\Psi_{I}\right)\right)=\left.\bigoplus_{i \in I^{\prime}} \mathcal{N}_{X} V_{i}\right|_{V_{I^{\prime}} \cap \operatorname{Im}\left(\Psi_{I}\right)} .
\end{gathered}
$$

We are only interested in regularizations near $\left(V_{i}\right)_{i \in S}$ and so we want a notion of equivalence to reflect this.

Definition 5.8. Two $\omega$-regularizations

$$
\left(\left(\rho_{i}\right)_{i \in S},\left(\Psi_{I}\right)_{I \subset S}\right), \quad\left(\left(\check{\rho}_{i}\right)_{i \in S},\left(\check{\Psi}_{I}\right)_{I \subset S}\right)
$$

for $\left(V_{i}\right)_{i \in S}$ are germ equivalent if there is an open set

$$
U_{I} \subset \operatorname{Dom}\left(\Psi_{I}\right) \cap \operatorname{Dom}\left(\check{\Psi}_{I}\right)
$$

containing $V_{I}$ so that $\left.\Psi_{I}\right|_{U_{I}}=\left.\check{\Psi}_{I}\right|_{U_{I}}$ and $\left.\rho_{i}\right|_{\Psi_{I}\left(U_{I}\right)}=\left.\check{\rho}_{i}\right|_{\Psi_{I}\left(U_{I}\right)}$ for each $i \in I \subset S$.

A real codimension 2 submanifold with an oriented normal bundle should be thought of as the differential geometric analogue of a smooth divisor in algebraic geometry. We wish to construct complex line bundles from such submanifolds in the same way that line bundles are constructed from Cartier divisors in algebraic geometry. The following line bundle associated to a codimension 2 submanifold $V$ of a manifold $X$ will depend on a choice of regularization $\Psi: \check{\mathcal{N}} \rightarrow X$ of $V$ and a complex structure $\mathbf{i}$ on $\mathcal{N}_{X} V$ and it will come with a canonical section $s_{V}: V \rightarrow \mathcal{O}_{X}(V)$ whose zero set is $V$. We define:

$$
\begin{gathered}
\mathcal{O}_{X}(V)=\left(\left.\left(\pi_{\mathcal{N}_{X} V}^{*} \mathcal{N}_{X}(V)\right)\right|_{\operatorname{Dom}(\Psi)} \sqcup(X-V) \times \mathbb{C}\right) / \sim, \\
\left.\left(\pi_{\mathcal{N}_{X} V}^{*} \mathcal{N}_{X}(V)\right)\right|_{\operatorname{Dom}\left(\Psi_{I}\right)} \ni(v, c v) \sim(\Psi(v), c) \in(X-V) \times \mathbb{C}, \quad \forall v \in \mathcal{N}_{X} V-V, c \in \mathbb{C} .
\end{gathered}
$$

The corresponding fibration is defined in the following natural way

$$
\begin{gathered}
\pi_{\mathcal{O}_{X}(V)}: \mathcal{O}_{X}(V) \longrightarrow X \\
\pi_{\mathcal{O}_{X}(V)}(v, w) \equiv \Psi(v), \quad \forall(v, w) \in \pi_{\mathcal{N}_{X} V}^{*}\left(\mathcal{N}_{X} V\right) \\
\pi_{\mathcal{O}_{X}(V)}(x, c) \equiv x, \quad \forall(x, c) \in(X-V) \times \mathbb{C} .
\end{gathered}
$$

This is a line bundle satisfying the following important canonical identities:

$$
\left.\mathcal{O}_{X}(V)\right|_{V}=\mathcal{N}_{X}(V),\left.\quad \mathcal{O}_{X}(V)\right|_{X-V}=(X-V) \times \mathbb{C} .
$$

We will call $\Psi$ the associated regularization. The canonical section $s_{V}: X \rightarrow \mathcal{O}_{X}(V)$ of this line bundle is defined as follows:

$$
s_{V}(x) \equiv\left\{\begin{array}{lll}
\left(\Psi^{-1}(x), \Psi^{-1}(x)\right) & \in\left(\left.\pi_{\mathcal{N}_{X} V}^{*} \mathcal{N}_{X}(V)\right|_{\operatorname{Dom}\left(\Psi_{I}\right)}\right. & \text { if } x \in \operatorname{Im}\left(\Psi_{I}\right) \\
(x, 1) & \in(X-V) \times \mathbb{C} & \text { if } x \in(X-V) .
\end{array}\right.
$$

We also define $\mathcal{O}_{X}(0) \equiv \mathcal{O}_{X} \equiv X \times \mathbb{C}$ to be the trivial bundle. This is also $\mathcal{O}_{X}(\emptyset)$ where $\emptyset$ is the empty submanifold. 
5.2. Trivializing Line Bundles. In the previous section, we constructed a line bundle from any codimension 2 submanifold with oriented boundary. As a result, we can construct line bundles from any SC divisor. In this subsection we show that if such a line bundle is trivial and if our SC divisor admits a regularization, then our line bundle admits a trivialization which behaves well with respect to this regularization. This trivialization will be used later to construct a map from a neighborhood of our SC divisor to $\mathbb{C}$ with nice parallel transport maps away from the singularities.

Let $(X, \omega)$ be a symplectic manifold and $\left(V_{i}\right)_{i \in S}$ an SC divisor on $X$ admitting an $\omega$ regularization

$$
\mathcal{R} \equiv\left(\left(\rho_{i}\right)_{i \in S},\left(\Psi_{I}\right)_{I \subset S}\right)
$$

as in Definition 5.7. Let $\left(m_{i}\right)_{i \in S}$ be natural numbers indexed by $S$. For all $i \in S$, let $\mathcal{O}_{X}\left(V_{i}\right)$ be the line bundle with associated regularization $\Psi_{i}$ and complex structure associated to the $\omega$-regularization $\left(\rho_{i}, \Psi_{i}\right)$. Recall that these have natural sections $s_{V_{i}}: X \rightarrow \mathcal{O}_{X}\left(V_{i}\right)$ as in Equation (5.11). Define $\mathcal{O}_{X}\left(\sum_{i} m_{i} V_{i}\right) \equiv \otimes_{i \in S} \mathcal{O}_{X}\left(V_{i}\right)^{\otimes m_{i}}$ and let

$$
s_{\left(m_{i}\right)_{i \in S}} \equiv \otimes_{i \in S} s_{V_{i}}^{\otimes m_{i}}
$$

be the canonical section of this bundle. Using the identity (5.10) we have

$$
\left.\mathcal{O}_{X}\left(V_{i}\right)\right|_{V_{I}}=\left.\mathcal{N}_{X} V_{i}\right|_{V_{I}}, \quad \forall i \in I \subset S,
$$

and hence we get the following maps:

$$
\begin{array}{r}
\Pi_{V_{i} ; I}: \mathcal{N}_{X} V_{I}=\left.\left.\bigoplus_{j \in I} \mathcal{N}_{X} V_{j}\right|_{V_{I}} \longrightarrow \mathcal{O}_{X}\left(V_{i}\right)\right|_{V_{I}}, \\
\left(v_{j}\right)_{j \in I} \longrightarrow\left\{\begin{array}{ll}
v_{i} & \text { if } i \in I \\
s_{V_{i}}(x) & \text { if } i \notin I
\end{array},\left.\quad \forall\left(v_{j}\right)_{j \in I} \in \mathcal{N}_{X} V_{I}\right|_{x}, x \in V_{I} .\right.
\end{array}
$$

Therefore we get a (not necessarily fiberwise linear) map:

$$
\begin{array}{r}
\Pi_{\left(m_{i}\right)_{i \in S}, I}:\left.\mathcal{N}_{X} V_{I} \longrightarrow \mathcal{O}_{X}\left(\sum_{i} m_{i} V_{i}\right)\right|_{V_{I}}, \\
\Pi_{\left(m_{i}\right)_{i \in S}, I}(v) \equiv \bigotimes_{i \in S} \Pi_{V_{i} ; I}(v)^{\otimes m_{i}} \quad \forall v \in \mathcal{N}_{X} V_{I} .
\end{array}
$$

One can think of the above map as a section of $\left.\mathcal{O}_{X}\left(\sum_{i} m_{i} V_{i}\right)\right|_{V_{I}}$ along with non-trivial infinitesimal information in the normal direction of $V_{I}$.

Below is a definition of a trivialization of $\mathcal{O}\left(\sum_{i} m_{i} V_{i}\right)$ with the property that locally around

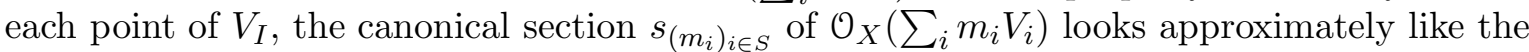
map $\left(z_{1}, \cdots, z_{n}\right) \rightarrow \prod_{i \in I}\left(z_{i} a\left(\left|z_{i}\right|\right) /\left|z_{i}\right|\right)^{m_{i}}$ in some local chart $z_{1}, \cdots, z_{n}$ where $I \subset\{1, \cdots, n\}$ and $a: \mathbb{R} \longrightarrow \mathbb{R}$ has the following graph:

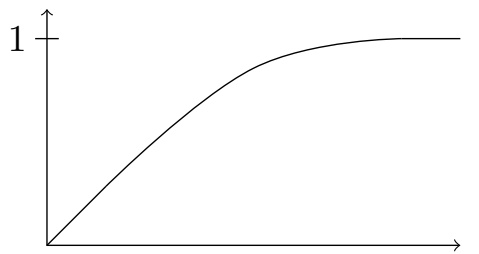

We need to use the above function $a$ to ensure that we have good dynamical properties (see Section [5.5). See [MTZ14a, Definition 3.8] for a related definition.

Definition 5.9. For each $r>0$, we define the radius $r$ tube of $V_{I}$ to be the set

$$
T_{r, I} \equiv \cap_{i \in I}\left\{x \in \operatorname{Im}\left(\Psi_{I}\right): \rho_{i}(x) \leq r\right\}
$$


over $V_{I}$. Let $B \subset X$ be any set. The tube radius of $\mathcal{R}$ along $B$ is the largest radius $r$ tube of $V_{I}$ along $B$ that can 'fit' inside the image of $\Psi_{I}$ for each $I \subset S$. More precisely, it is the supremum of all $r \geq 0$ with the property that $T_{r, I} \cap\left(\left.\operatorname{Im}\left(\Psi_{I}\right)\right|_{x}\right)$ is a compact subset of $X$ for all $x \in B \cap V_{I}$ and $I \subset S$.

Let $U \subset X$ be an open set. Now suppose that the tube radius of $\mathcal{R}$ along $U$ is positive and let $R>0$ be any constant smaller than the tube radius and also smaller than 1 . We let $a_{R}:[0, \infty) \longrightarrow[0, \infty)$ be a smooth function satisfying:

(1) $a_{R}^{\prime}(x)>0$ for $x \in[0,3 R / 4)$,

(2) $a_{R}(x)=x$ for $x \leq R / 4$,

(3) $a_{R}(x)=1$ for $x \geq 3 R / 4$.

A bundle trivialization:

$$
\Phi \equiv\left(\pi, \Phi_{2}\right): \mathcal{O}_{X}\left(\sum_{i} m_{i} V_{i}\right) \longrightarrow X \times \mathbb{C}
$$

is radius $R$ compatible with $\mathcal{R}$ along $U \cap V_{I}$ if

$$
\begin{array}{r}
\Phi_{2}\left(s_{\left(m_{i}\right)_{i \in S}}(x)\right)=\Phi_{2}\left(\Pi_{\left(m_{i}\right)_{i \in S}, I}\left(\Psi_{I}^{-1}(x)\right)\right) \prod_{i \in I}\left(\frac{\sqrt{a_{R}\left(\rho_{i}(x)\right)}}{\sqrt{\rho_{i}(x)}}\right)^{m_{i}}, \\
\forall x \in T_{R, I} \cap\left(\left.\operatorname{Im}\left(\Psi_{I}\right)\right|_{V_{I} \cap U-\cup_{i \in S-I} T_{\frac{3 R}{4}, i}}\right) .
\end{array}
$$

and where the norm of the linear map:

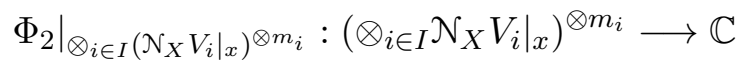

is equal to 1 for all $x \in V_{I} \cap U-\cup_{i \in S-I} T_{\frac{3 R}{4}, i}$ using the identification (5.10).

We say that $\Phi$ is radius $R$ compatible with $\mathcal{R}$ along $U$ if it is radius $R$ compatible with $\mathcal{R}$ along $U \cap V_{I}$ for each $I \subset S$. It is compatible with $\mathcal{R}$ along $U$ if it is radius $R$ compatible with $\mathcal{R}$ along $U$ for some $R$ smaller than the tube radius of $\mathcal{R}$.

One should think of Equation (5.15) as saying that the trivialization $\Phi$ identifies the canonical section of $\mathcal{O}_{X}\left(\sum_{i} m_{i} V_{i}\right)$ with the 'infinitesimal' section $\Pi_{\left(m_{i}\right)_{i \in S}, I}$ multiplied by a particular factor near $V_{I}$. This particular factor that we are multiplying by is actually equal to 1 if we are very near $V_{I}$. Note also that Equation (5.15) tells us that the norm of $\Phi_{2}\left(s_{\left(m_{i}\right)_{i \in S}}(x)\right)$ only depends on $\left(\rho_{i}(x)\right)_{i \in I}$ and if $\rho_{i}(x) \geq 3 R / 4$ for some $i \in I$ then this norm does not depend on $\rho_{i}(x)$ for all $x \in \operatorname{Im}\left(\Psi_{I}\right)-\cup_{i \in S-I} T_{3 R / 4, i}$. As a result, the above definition is consistent with the stated norm property of the linear map (5.16).

Lemma 5.10. Suppose $\mathcal{O}_{X}\left(\sum_{i} m_{i} V_{i}\right)$ admits a trivialization $\Phi$ and let $U \subset X$ be a relatively compact open set. Then there is a trivialization of $\mathcal{O}_{X}\left(\sum_{i} m_{i} V_{i}\right)$ compatible with $\mathcal{R}$ along $U$ which is homotopic to $\Phi$ through trivializations of $\mathcal{O}_{X}\left(\sum_{i} m_{i} V_{i}\right)$.

Proof. Just as in the proof of [MTZ14a, Definition 3.9] we will proceed by induction on the strata of $\cup_{j} V_{j}$. Before we do this though we will need to construct certain natural maps that identify $\Pi_{\left(m_{i}\right)_{i \in S} ; I}$ with $s_{\left(m_{i}\right)_{i \in S}}$ near $V_{I}$. Define

$$
\begin{gathered}
\left.N_{i, I} \equiv \operatorname{Dom}\left(\Psi_{i}\right)\right|_{V_{i} \cap \operatorname{Im}\left(\Psi_{I \cup i}\right)} \stackrel{\sqrt[5.8]{=}}{=} \mathfrak{D} \Psi_{I \cup i ; i}\left(\operatorname{Dom}\left(\Psi_{I \cup i}\right)\right) \\
W_{i ; I} \equiv \Psi_{I}\left(\left.\operatorname{Dom}\left(\Psi_{I}\right)\right|_{V_{I}-V_{i}}\right) \\
D_{i ; I} \equiv W_{i ; I} \cup \Psi_{i}\left(N_{i ; I}\right)
\end{gathered}
$$

for all $i \in S, I \subset S$. By Equation (5.9), we have the natural identification:

$$
\left.\mathcal{O}_{X}\left(V_{i}\right)\right|_{\Psi_{i}\left(N_{i ; I}\right)}=\left.\pi_{\mathcal{N}_{X} V_{i}}^{*} \mathcal{N}_{X}\left(V_{i}\right)\right|_{N_{i ; I}}
$$


and by (5.6) and (5.10) we have the identity

$$
\left.\mathcal{O}_{X}\left(V_{i}\right)\right|_{W_{i ; I}}=W_{i: I} \times \mathbb{C}
$$

for all $i \in S$ and $I \subset S$. By using the natural projections

$$
\operatorname{Pr}_{I ; I^{\prime}}:\left.\mathcal{N}_{X} V_{I} \longrightarrow \mathcal{N}_{X} V_{I^{\prime}}\right|_{V_{I}}, \quad \forall I^{\prime} \subset I \subset S
$$

we have a map:

$$
\widehat{\Pi}_{V_{i} ; I}:\left.\left.\mathcal{O}_{X}\left(V_{i}\right)\right|_{D_{I ; i}} \longrightarrow \mathcal{O}_{X}\left(V_{i}\right)\right|_{V_{I}}
$$

for all $i \in S$ and $I \subset S$ whose restriction to $\Psi_{i}\left(N_{i ; I}\right)$ is defined by the equation

$$
\begin{array}{r}
\widehat{\Pi}_{V_{i} ; I}(v, w) \equiv\left(\operatorname{Pr}_{I \cup i ; i}\left(\mathfrak{D} \Psi_{I \cup i ; i}^{-1}(v)\right), \operatorname{Pr}_{I \cup i ; i}\left(\mathfrak{D} \Psi_{I \cup i ; i}^{-1}(w)\right)\right) \\
\left.\forall(v, w) \in\left(\pi_{\mathcal{N}_{X} V_{i}}^{*} \mathcal{N}_{X}\left(V_{i}\right)\right)\right|_{N_{i ; I}}
\end{array}
$$

by using the identity (5.17) and whose restriction to $W_{i ; I}$ is defined by

$$
\widehat{\Pi}_{V_{i} ; I}(x, c) \equiv\left(\pi_{\mathcal{N}_{X} V_{I}}\left(\Psi_{I}^{-1}(x)\right), c\right) \in\left(V_{I}-V_{i}\right) \times \mathbb{C}, \quad \forall(x, c) \in W_{i: I} \times \mathbb{C}
$$

by using the identity (5.18). One should think of this map as a way of canonically identifying the bundle $\mathcal{O}_{X}\left(V_{i}\right)$ near $V_{I}$ with its pullback along the natural projection map from $D_{i ; I}$ to $V_{I}$ induced by $\pi_{\mathcal{N}_{X} V_{I}} \circ \Psi_{I}^{-1}$. By (5.9) and (5.11),

$$
\left.\Pi_{V_{i} ; I}\right|_{\Psi_{I}^{-1}\left(D_{i ; I}\right)}=\widehat{\Pi}_{V_{i} ; I} \circ s_{V_{i}} \circ \Psi_{I} \quad \forall i \in S, I \subset S .
$$

Also by (5.8) and the fact that $\mathfrak{D} \Psi_{I ; I^{\prime}}$ is a product Hermitian isomorphism for all $I^{\prime} \subset I \subset S$,

$$
\left.\widehat{\Pi}_{V_{i} ; I} \circ \widehat{\Pi}_{V_{i} ; I^{\prime}}\right|_{D_{I ; i}}=\widehat{\Pi}_{V_{i} ; I} \quad \forall i \in S, I^{\prime} \subset I \subset S .
$$

We can define similar maps for the line bundle $\mathcal{O}_{X}\left(\sum_{i} m_{i} V_{i}\right)$ in the following way

$$
\begin{array}{r}
\widehat{\Pi}_{\left(m_{i}\right)_{i \in S} ; I}:\left.\left.\mathcal{O}_{X}\left(\sum_{i} m_{i} V_{i}\right)\right|_{\cap_{i \in S} D_{i ; I}} \longrightarrow \mathcal{O}_{X}\left(\sum_{i} m_{i} V_{i}\right)\right|_{V_{I}} \\
\widehat{\Pi}_{\left(m_{i}\right)_{i \in S} ; I}\left(\otimes_{i \in S, j \in\left\{1, \cdots, m_{i}\right\}} v_{i, j}\right)=\otimes_{i \in S, j \in\left\{1, \cdots, m_{i}\right\}} \widehat{\Pi}_{V_{i} ; I}\left(v_{i, j}\right) \quad \forall I \subset S .
\end{array}
$$

Equations (5.19) and (5.20) give us the following equations:

$$
\begin{array}{r}
\left.\Pi_{\left(m_{i}\right)_{i \in S} ; I}\right|_{\Psi_{I}^{-1}\left(\cap_{i \in S} D_{i ; I}\right)}=\left.\widehat{\Pi}_{\left(m_{i}\right)_{i \in S} ; I} \circ s_{\left(m_{i}\right)_{i \in S}} \circ \Psi_{I}\right|_{\cap_{i \in S} D_{i ; I}} \quad \forall I \subset S \\
\left.\widehat{\Pi}_{\left(m_{i}\right)_{i \in S} ; I} \circ \widehat{\Pi}_{\left(m_{i}\right)_{i \in S} ; I^{\prime}}\right|_{\cap_{i \in S} D_{i ; I}}=\widehat{\Pi}_{\left(m_{i}\right)_{i \in S} ; I} \quad \forall I^{\prime} \subset I \subset S .
\end{array}
$$

Using these equations, we will now prove our lemma by induction on the set of subsets of $S$. Let $\preceq$ be a total order on the set of subsets of $S$ with the property that if $\left|I^{\prime}\right|<|I|$ then $I \preceq I^{\prime}$. We write $I \prec I^{\prime}$ when $I \preceq I^{\prime}$ and $I \neq I^{\prime}$. Since $U$ is relatively compact, the tube radius of $\mathcal{R}$ along $U$ is positive and hence we can choose any constant $R>0$ smaller than this tube radius.

Suppose that there is some $I^{*} \subset S$ and a trivialization

$$
\Phi^{\prec} \equiv\left(\pi, \Phi_{2}^{\prec}\right): \mathcal{O}_{X}\left(\sum_{i} m_{i} V_{i}\right) \longrightarrow X \times \mathbb{C}
$$

which is radius $R$ compatible with $\mathcal{R}$ along $U \cap V_{I}$ for all $I \prec I^{*}$ and which is isotopic to $\Phi$ through trivializations of $\mathcal{O}_{X}\left(\sum_{i} m_{i} V_{i}\right)$. We now wish to modify the trivialization $\Phi^{\prec}$ so that these properties hold for all $I \preceq I^{*}$. Let $T_{r, I}$ be the radius $r$ tube of $V_{I}$ as in Equation (5.14) and define $L_{r}^{\prec} \equiv \cup_{I \prec I^{*}} T_{r, I}$.

First of all, let

$$
g: V_{I^{*}}-L_{\frac{3 R}{4}}^{\prec} \longrightarrow(0, \infty)
$$


be a smooth function whose value at $x \in V_{I^{*}}-L_{\frac{3 R}{4}}^{\prec}$ is equal to the norm of the linear map

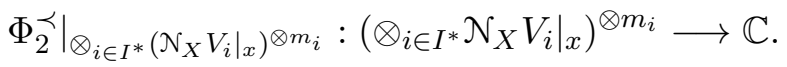

Since the map $\Pi_{V_{i} ; I}$ restricted to each fiber of $\operatorname{Pr}_{I ; I-i}$ is an isometry for all $i \in I \subset S$, we get that $g(x)=1$ for all $x \in V_{I^{*}} \cap\left(L_{R}^{\prec}-L_{\frac{3 R}{4}}^{\prec}\right)$ by our induction hypothesis. Combining this with the fact that $a_{R}(s)=1$ for all $s \geq 3 R / 4$, we can choose a smooth function $f$ : $X \longrightarrow(0, \infty)$ which is equal to 1 in the region $L_{R}^{\prec}$ and equal to $\left(\pi_{\mathcal{N}_{X} V_{I^{*}}} \circ \Psi_{I^{*}}^{-1}\right)^{*}\left(\frac{1}{g}\right)$ inside $T_{R, I^{*}} \cap \Psi_{I^{*}}\left(\left.\operatorname{Dom}\left(\Psi_{I^{*}}\right)\right|_{V_{I^{*}}-L_{\frac{3 R}{4}}^{\prec}}\right)$. This implies that the norm of the linear map:

$$
\left.f(x) \Phi_{2}^{\prec}\right|_{\otimes_{i \in I}\left(\left.\mathcal{N}_{X} V_{i}\right|_{x}\right)^{\otimes m_{i}}}:\left(\left.\otimes_{i \in I} \mathcal{N}_{X} V_{i}\right|_{x}\right)^{\otimes m_{i}} \longrightarrow \mathbb{C}
$$

is 1 for all $I \preceq I^{*}$. Hence Equation (5.16) holds for $f \Phi_{2}^{\prec}$ for all $I \preceq I^{*}$.

We now wish to modify $f \Phi^{\prec}$ so that Equation (15.15) for this new trivialization holds as well. We let

$$
\begin{array}{r}
\Phi^{=} \equiv\left(\pi, \Phi_{2}^{=}\right):\left.\mathcal{O}_{X}\left(\sum_{i} m_{i} V_{i}\right)\right|_{\operatorname{Im}\left(\Psi_{I^{*}}\right)} \longrightarrow X \times \mathbb{C}, \\
\Phi^{=}(v) \equiv f(x) \Phi^{\prec}\left(\widehat{\Pi}_{\left(m_{i}\right)_{i \in S} ; I^{*}}(v)\right) \prod_{i \in I^{*}}\left(\frac{\sqrt{a_{R}\left(\rho_{i}(x)\right)}}{\sqrt{\rho_{i}(x)}}\right)^{m_{i}}, \\
\left.\forall v \in \mathcal{O}_{X}\left(\sum_{i} m_{i} V_{i}\right)\right|_{x}, \quad x \in \operatorname{Im}\left(\Psi_{I^{*}}\right) .
\end{array}
$$

be a smooth trivialization. Equation (5.22) combined with the fact that $\Phi_{2}^{\prec}$ is radius $R$ compatible with $\mathcal{R}$ along $U \cap V_{I}$ for all $I \prec I^{*}$ implies that $\Phi_{2}^{\prec}$ is radius $R$ compatible with $\mathcal{R}$ along $U \cap\left(L_{R}^{\prec}-L_{\frac{3 R}{4}}^{\prec}\right) \cap V_{I^{*}}$. Hence by Equation (5.24), we have

$$
\Phi_{2}^{\prec}(v)=\Phi_{2}^{=}(v), \quad \forall v \in T_{R, I^{*}} \cap\left(L_{R}^{\prec}-L_{\frac{3 R}{4}}^{\prec}\right) .
$$

Combining this with the fact that $T_{R, I^{*}}$ deformation retracts on to $V_{I^{*}} \cup\left(T_{R, I^{*}} \cap L_{R}^{\prec}\right)$, we can construct a smooth trivialization

$$
\Phi \preceq \equiv\left(\pi, \Phi_{2}^{\prec}\right): \mathcal{O}_{X}\left(\sum_{i} m_{i} V_{i}\right) \longrightarrow X \times \mathbb{C}
$$

homotopic to $\Phi^{\prec}$ so that

$$
\left.\Phi \preceq\right|_{L_{R}^{\prec}}=\left.\Phi^{\prec}\right|_{L_{R}^{\prec}} \text { and }\left.\Phi \preceq\right|_{T_{R, I^{*}}}=\left.\Phi^{=}\right|_{T_{R, I^{*}}}
$$

Equations (5.21), (5.24) and (5.25) tell us that $\Phi \preceq$ is radius $R$ compatible with $\mathcal{R}$ along $U \cap V_{I^{*}}$. Also since $\Phi \preceq=\Phi^{\prec}$ along $L_{R}^{\prec}$, we get that $\Phi \preceq$ is radius $R$ compatible with $\mathcal{R}$ along $U \cap V_{I}$ for all $I \prec I^{*}$. Hence $\Phi \preceq$ is radius $R$ compatible with $\mathcal{R}$ along $U \cap V_{I}$ for all $I \preceq I^{*}$.

Because the norm of the linear map (5.23) is 1 for all $I \preceq I^{*}$, we have by Equations (5.24) and (5.25) that the norm of the linear map

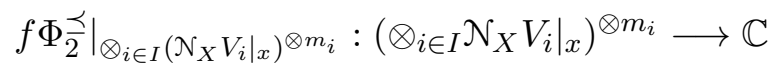

is equal to 1 for all $I \preceq I^{*}$. Hence we are done by induction. 
5.3. Links of Divisors and Open Books. In this subsection, we first give a purely symplectic definition of a divisor which looks like the resolution divisors of a log resolution of an isolated hypersurface singularity. We then construct the 'link' of this resolution divisor, which corresponds to the embedded link of our isolated singularity. Finally we construct an open book decomposition of this resolution divisor corresponding the Milnor open book of our hypersurface singularity.

We have the following definition from [McL12a].

Definition 5.11. Let $(X, \omega)$ be a symplectic manifold of dimension $2 n$ and let $\theta \in \Omega^{1}(X-K)$ satisfy $d \theta=\left.\omega\right|_{X-K}$ for some compact $K \subset X$. Suppose that $K$ admits an open neighborhood $U$ which deformation retracts onto $K$. Let $\rho: X \longrightarrow[0,1]$ be a smooth function equal to 0 along $K$ and equal to 1 outside a compact subset of $U$. Then the dual

$$
c(\omega, \theta) \in H_{2 n-2}(K ; \mathbb{R})=H_{2 n-2}(U ; \mathbb{R})
$$

of $(\omega, \theta)$ is defined to be the Lefschetz dual of $\left.(\omega-d(\rho \theta))\right|_{U} \in H_{c}^{2}(U)$.

Now suppose that $K=\cup_{i \in S} V_{i}$ where $\left(V_{i}\right)_{i \in S}$ is an SC divisor and each $V_{i}$ is connected and compact. Then a Mayor-Vietoris argument tells us that $H_{2 n-2}\left(\cup_{i \in S} V_{i} ; \mathbb{R}\right)$ is freely generated by the fundamental classes $\left[V_{i}\right]$ and hence there are unique real numbers $\left(w_{i}\right)_{i \in S}$ so that $c(\omega, \theta)=-\sum_{i} w_{i}\left[V_{i}\right]$. The wrapping number of $\theta$ around $V_{j}$ is defined to be $w\left(\theta, V_{j}\right) \equiv w_{j}$.

The wrapping number does not depend on the choice of neighborhood $U$ or bump function $\rho$. We can calculate the wrapping number $w\left(\theta, V_{j}\right)$ in the following way (See the discussion after Definition 5.4 in [McL16]): Let $D \subset X$ be a small symplectically embedded disk with polar coordinates $(r, \vartheta)$ which intersects $V_{i}$ positively once at $0 \in D$ and does not intersect $V_{j}$ for all $j \in S-i$. Then the wrapping number $w_{i}$ is the unique number so that $\left(w_{i} / 2 \pi\right) d \vartheta$ is cohomologous to

$$
\left.\theta\right|_{D-0}-\frac{1}{2} r^{2} d \vartheta
$$

inside $H^{1}(D-0 ; \mathbb{R})$. The computation of the wrapping number using the disk $D$ above enables us to define wrapping numbers in the case when each $V_{i}$ is properly embedded but not necessarily compact. We will need this broader definition of wrapping number in the proof of Lemma 5.23 below.

The next definition is supposed to be a way of describing a log resolution of a pair $\left(\mathbb{C}^{n+1}, f^{-1}(0)\right.$ ) (as in Definition 2.1) in a symplectic way. The key motivating example for such a definition is given in Example 5.14 below.

Definition 5.12. A resolution divisor is a pair $\left(X,\left(V_{i}\right)_{i \in S}\right)$ where $\left(V_{i}\right)_{i \in S}$ are transversally intersecting properly embedded codimension 2 submanifolds of a manifold $X$ so that there is a unique element $\star_{S} \in S$ with the property that $V_{\star_{S}}$ is non-compact and $V_{i}$ is compact for all $i \in S-\star_{S}$. We also require that $\cup_{i} V_{i}$ is connected and that $V_{i}$ is connected for each $i \in S-\star_{S}$ (although $V_{\star_{S}}$ is allowed to be disconnected).

A model resolution is a triple $\left(\mathcal{O}_{X}\left(\sum_{i \in S} m_{i} V_{i}\right), \Phi, \theta\right)$ where $X$ is a manifold, $\left(V_{i}\right)_{i \in S}$ are codimension 2 submanifolds, $\theta \in \Omega^{1}\left(X-\cup_{i \in S-\star_{S}} V_{i}\right)$ and $\Phi$ is a trivialization of

$$
\mathcal{O}_{X}\left(\sum_{i \in S} m_{i} V_{i}\right) \equiv \otimes_{i \in S} \mathcal{O}_{X}\left(V_{i}\right)^{\otimes m_{i}}
$$

where $\left(m_{i}\right)_{i \in S}$ are positive integers satisfying:

(1) $\left(X,\left(V_{i}\right)_{i \in S}\right)$ is a resolution divisor as above,

(2) $d \theta=\left.\omega\right|_{X-\cup_{i \in S-\star_{S}} V_{i}}$ for some symplectic form $\omega$ on $X$,

(3) $\left(V_{i}\right)_{i \in S}$ is an $\mathrm{SC}$ divisor with respect to $\omega$, 
(4) $m_{\star_{S}}=1$ and the wrapping number $w\left(\theta, V_{i}\right)$ is positive for all $i \in S-\star_{S}$.

The form $\omega$ is called the symplectic form associated to $\left(\mathcal{O}_{X}\left(\sum_{i \in S} m_{i} V_{i}\right), \Phi, \theta\right)$. A grading on this model resolution is a grading on $\left(X-\cup_{i \in S-\star_{S}} V_{i}, \omega\right)$.

Definition 5.13. Two model resolutions

$$
Y \equiv\left(\mathcal{O}_{X}\left(\sum_{i \in S} m_{i} V_{i}\right), \Phi, \theta\right), \quad \widehat{Y} \equiv\left(\mathcal{O}_{\widehat{X}}\left(\sum_{i \in \widehat{S}} \widehat{m}_{i} \widehat{V}_{i}\right), \widehat{\Phi}, \widehat{\theta}\right)
$$

are isotopic if there is

- a bundle isomorphism $\widetilde{\Psi}: \mathcal{O}_{X}\left(\sum_{i \in S} m_{i} V_{i}\right) \longrightarrow \mathcal{O}_{\widehat{X}}\left(\sum_{i \in \widehat{S}} \widehat{m}_{i} \widehat{V}_{i}\right)$ covering a diffeomorphism $\Psi: X \longrightarrow \widehat{X}$

- a bijection $\iota: S \longrightarrow \widehat{S}$ sending $\star_{S}$ to $\star_{\widehat{S}}$,

- a smooth family of 1 -forms $\left(\theta_{t} \in \Omega^{1}\left(X-\cup_{i \in S-\star_{S}} V_{i}\right)\right)_{t \in[0,1]}$ joining $\theta$ and $\Psi^{*} \widehat{\theta}$ and trivializations $\left(\Phi_{t}\right)_{t \in[0,1]}$ of $\mathcal{O}_{X}\left(\sum_{i \in S} m_{i} V_{i}\right)$ joining $\Phi$ and $\widehat{\Phi} \circ \widetilde{\Psi} \circ\left(\Psi^{-1} \times \operatorname{id}_{\mathbb{C}}\right)$

so that $m_{i}=\widehat{m}_{\iota(i)}$ and $\Psi\left(V_{i}\right)=\widehat{V}_{\iota(i)}$ for all $i \in S$ and $Y_{t} \equiv\left(\mathcal{O}_{X}\left(\sum_{i \in S} m_{i} V_{i}\right), \Phi_{t}, \theta_{t}\right)$ is a model resolution for all $t \in[0,1]$.

These model resolutions are graded isotopic if they are isotopic as above with the additional property that the model resolutions $Y_{t}$ all admit gradings that smoothly depend on $t \in[0,1]$ and where the grading on $Y_{0}$ coincides with the grading on $Y$ and the grading on $Y_{1}$ coincides with the grading on $\widehat{Y}$ under the identification $\Psi$.

We will now give an example of a model resolution.

Example 5.14. Let $f: \mathbb{C}^{n+1} \longrightarrow \mathbb{C}$ be a polynomial with an isolated singularity at 0 and let $U \subset \mathbb{C}^{n+1}$ be an open set containing 0 so that $\left.f\right|_{U-0}$ is regular. Let $\pi: Y \longrightarrow \mathbb{C}^{n+1}$ be a log resolution of $\left(\mathbb{C}^{n+1}, f^{-1}(0)\right)$ obtained by a sequence of blowups along smooth loci and define $X \equiv \pi^{-1}(U)$ (such a resolution exists by [Hir64]). Let $\left(E_{j}\right)_{j \in S}$ be the resolution divisors of this resolution and let $E_{\star_{S}} \subset X$ be the proper transform of $f^{-1}(0) \cap U$. Because such a resolution is obtained by a sequence of blowups along smooth loci, there are positive integers $\left(w_{j}\right)_{j \in S-\star_{S}}$ so that $A \equiv-\sum_{i \in S-\star_{S}} w_{i} E_{i}$ is ample on $X$. By the divisor line bundle correspondence, let $L \longrightarrow X$ be the corresponding ample line bundle with a meromorphic section satisfying $(s)=A$. Choose a Hermitian metric $\|\cdot\|$ on $L$ whose curvature form is a positive 1-1 form. Define $\theta=-d^{c} \ln (\|s\|)$. The 2-form $d \theta$ extends uniquely to a Kähler form $\omega$ on $X$. The wrapping number of $\theta$ around $E_{j}$ is $w_{j}$ for all $j \in S-\star_{S}$.

We also let $m_{i} \in \mathbb{N}_{>0}$ be the multiplicity of $f$ along $E_{i}$ for all $i \in S$. Since $\sum_{i \in S} m_{i} E_{i}$ is the divisor defined by $\left.f \circ \pi\right|_{X}$ we get, by the divisor line bundle correspondence, that the holomorphic line bundle $\mathcal{O}_{X}\left(\sum_{i \in S} m_{i} E_{i}\right)$ has an induced trivialization $\Phi: \mathcal{O}_{X}\left(\sum_{i \in S} m_{i} E_{i}\right) \cong$ $X \times \mathbb{C}$ so that the section $s$ corresponding to the holomorphic function $\left.f \circ \pi\right|_{X}$ satisfies $p r_{2} \circ \Phi \circ s=\left.f \circ \pi\right|_{X}$ where $p r_{2}: X \times \mathbb{C} \longrightarrow \mathbb{C}$ is the natural projection map. Then $\left(\mathcal{O}_{X}\left(\sum_{i \in S} m_{i} E_{i}\right), \Phi, \theta\right)$ is a model resolution called a model resolution associated to $f$.

Such a resolution also has a grading as follows: Let $\check{X} \equiv X-\cup_{i \in S-\star_{S}} E_{i}$. Since

$$
\left.\pi\right|_{\check{X}}: \check{X} \longrightarrow U-0 \subset \mathbb{C}^{n+1}
$$

is a biholomorphism we get a canonical holomorphic trivialization

$$
\Phi: T \check{X} \longrightarrow \check{X} \times \mathbb{C}^{n+1}
$$


as a unitary vector bundle coming from the trivialization on $T \mathbb{C}^{n+1}$. The grading on $T \check{X}$ is equal to the trivial grading on $\check{X} \times \mathbb{C}^{n+1}$ pulled back by $\Phi$. We will call this the standard grading.

We now wish to associate a pair of contact manifolds with normal bundle data to a model resolution. In the case of Example [5.14, this is the link of our singularity (as defined in the introduction) with some additional data.

Definition 5.15. Let $\left(X,\left(V_{i}\right)_{i \in S}\right)$ be a resolution divisor. A tuple of regularizations

$$
\left(\Psi_{i}\right)_{i \in S-\star_{S}}
$$

is compatible with $V_{\star_{S}}$ if $\Psi_{i}$ is a regularization of $V_{i}$ for each $i \in S-\star_{S}$ and

$$
\Psi_{i}\left(\left.\operatorname{Dom}\left(\Psi_{i}\right)\right|_{{\star}_{S} \cap V_{i}}\right) \subset V_{\star_{S}} \text { for all } i \in S-\star_{S} \text {. }
$$

In other words, $\Psi_{i}$ restricted to $\left.\operatorname{Dom}\left(\Psi_{i}\right)\right|_{{\star S}_{S} \cap V_{i}}$ is a regularization of $V_{i} \cap V_{\star_{S}}$ inside $V_{\star_{S}}$ for all $i \in S-\star_{S}$.

Definition 5.16. Let $\Psi, \check{\Psi}$ be regularizations of a submanifold $V$ of a manifold $X$. Then a smooth family of regularizations $\Psi_{t}$ of $V$ connects $\Psi$ and $\check{\Psi}$ if $\Psi$ is germ equivalent to $\Psi_{0}$ and $\check{\Psi}$ is germ equivalent to $\Psi_{1}$.

Lemma 5.17. Let $\left(X,\left(V_{i}\right)_{i \in S}\right)$ be a resolution divisor. For any two tuples of regularizations $\left(\Psi_{i}\right)_{i \in S-\star_{S}},\left(\check{\Psi}_{i}\right)_{i \in S-\star_{S}}$ compatible with $V_{\star_{S}}$, there is a smooth family of such regularizations

$$
\left(\Psi_{i}^{t}\right)_{i \in S-\star_{S}}, \quad t \in[0,1]
$$

compatible with $V_{\star_{S}}$ which connects $\left(\Psi_{i}\right)_{i \in S-\star_{S}}$ and $\left(\check{\Psi}_{i}\right)_{i \in S-\star_{S}}$.

Proof. Choose a metric making $V_{\star_{S}}$ into a totally geodesic submanifold. Define $T^{r} X \subset T X$ to be the set of tangent vectors of length at most $r$. Fix $i \in S$. Choose a relatively compact neighborhood $W_{i}$ of $V_{i}$ in $X$ and let $\delta>0$ small enough so that the exponential map restricted to $T_{w}^{\delta} X$ is a diffeomorphism onto its image for all $w$ in $W_{i}$. Let $\widetilde{W}_{i} \subset \Psi_{i}^{-1}\left(W_{i}\right) \cap\left(\check{\Psi}_{i}\right)^{-1}\left(W_{i}\right)$ be a small enough neighborhood of $V_{i}$ so that the distance $d(v)$ between $\Psi_{i}(v)$ and $\check{\Psi}_{i}(v)$ is less than $\delta$ for all $v \in \widetilde{W}_{i}$. Now let $\gamma_{v}:[0, d(v)] \longrightarrow X$ be the unique geodesic of length $<\delta$ joining $\Psi_{i}(v)$ and $\check{\Psi}_{i}(v)$ for all $v \in \widetilde{W}_{i}$. Define

$$
\widetilde{\Psi}_{i}^{t}: \widetilde{W}_{i} \longrightarrow X, \quad \widetilde{\Psi}_{i}^{t}(v) \equiv \gamma_{v}(t d(v)) \quad \forall t \in[0,1] .
$$

Since $d_{v} \Psi$ and $d_{v} \check{\Psi}$ from Definition 5.2 are the both identity map, we get that $d_{v} \widetilde{\Psi}_{i}^{t}$ is also the identity map for all $v \in V_{i}$. Hence there is a neighborhood $\widetilde{W}_{i}^{\prime} \subset \widetilde{W}_{i}$ of $V_{i}$ so that $\left.\Psi_{i}^{t} \equiv \widetilde{\Psi}_{i}^{t}\right|_{\widetilde{W}^{\prime}}$ is a diffeomorphism onto its image for all $t \in[0,1]$. Hence $\left(\Psi_{i}^{t}\right)_{t \in[0,1]}$ is a smooth family of regularizations compatible with $V_{\star_{S}}$ which connects $\Psi_{i}$ and $\check{\Psi}_{i}$. Therefore $\left(\Psi_{i}^{t}\right)_{i \in S-\star_{S}}, t \in[0,1]$ connects $\left(\Psi_{i}\right)_{i \in S-\star_{S}}$ and $\left(\check{\Psi}_{i}\right)_{i \in S-\star_{S}}$.

Definition 5.18. Let $X$ be a smooth manifold and let $\left(V_{i}\right)_{i \in \check{S}}$ be transversely intersecting compact codimension 2 submanifolds of $X$. A smooth function

$$
f: X-\cup_{i \in \check{S}} V_{i} \longrightarrow \mathbb{R}
$$

is compatible with $\left(V_{i}\right)_{i \in \check{S}}$ if there is

- a regularization $\Psi_{i}$ of $V_{i}$,

- a real number $b_{i}>0$ and

- a smooth function $q_{i}: \operatorname{Dom}\left(\Psi_{i}\right) \longrightarrow[0,1]$ equal to the square of some norm on $\mathcal{N}_{X} V_{i}$ near $V_{i}$, equal to 1 outside a compact subset of $\operatorname{Dom}\left(\Psi_{i}\right)$ and non-zero on $\operatorname{Dom}\left(\Psi_{i}\right)-V_{i}$ 
for each $i \in S$ and a smooth function $\tau: X \longrightarrow \mathbb{R}$ so that

$$
f=\sum_{i \in \check{S}} b_{i} \log \left(q_{i} \circ \Psi_{i}^{-1}\right)+\tau
$$

where $\log \left(q_{i} \circ \Psi_{i}^{-1}\right)$ is defined to be 0 outside $\operatorname{Im}\left(\Psi_{i}\right)$ for each $i \in S$. We will call the regularizations $\left(\Psi_{i}\right)_{i \in S-\star_{S}}$ associated regularizations of $f$.

Now suppose that we have an additional smooth submanifold $V_{\star_{S}}$ of $X$ so that $\left(V_{i}\right)_{i \in S}$ becomes a resolution divisor where $S=\check{S} \sqcup\left\{\star_{S}\right\}$. We say that $f$ is compatible with $\left(V_{i}\right)_{i \in S}$ if it is compatible with $\left(V_{i}\right)_{i \in S-\star_{S}}$ as above with the additional property that the associated regularizations of $f$ are compatible with $V_{\star_{S}}$. As a consequence of this we have that $\left.f\right|_{V_{\star_{S}}}$ is compatible with $\left(V_{i} \cap V_{\star_{S}}\right)_{i \in S-\star_{S}}$.

We say that $f$ is strongly compatible with $\left(V_{i}\right)_{i \in S}$ if in addition $\tau=0$.

Lemma 5.19. Let $\left(X,\left(V_{i}\right)_{i \in S}\right)$ be a resolution divisor and let

$$
f, g: X-\cup_{i \in S-\star_{S}} V_{i} \longrightarrow \mathbb{R}
$$

be a pair of smooth functions compatible with $\left(V_{i}\right)_{i \in S}$. Then there is a smooth family of functions

$$
f_{t}: X-\cup_{i \in S-\star_{S}} V_{i} \longrightarrow \mathbb{R}, \quad t \in[0,1]
$$

compatible with $\left(V_{i}\right)_{i \in S}$ so that $f_{0}=f$ and $f_{1}=g$.

Proof. For all $i \in S-\star_{S}$, there are regularizations $\Psi_{i}, \check{\Psi}_{i}$ of $V_{i}$, smooth functions

$$
q_{i}: \operatorname{Dom}\left(\Psi_{i}\right) \longrightarrow[0,1], \quad \check{q}_{i}: \operatorname{Dom}\left(\check{\Psi}_{i}\right) \longrightarrow[0,1]
$$

equal to the square of a norm near $V_{i}$, equal to 1 outside a compact set and non-zero outside $V_{i}$, real numbers $b_{i}, \check{b}_{i}>0$ and smooth functions $\tau, \check{\tau}: X \longrightarrow \mathbb{R}$ so that

$$
f=\sum_{i \in S-\star_{S}} b_{i} \log \left(q_{i} \circ \Psi_{i}^{-1}\right)+\tau \text { and } g=\sum_{i \in S-\star_{S}} \check{b}_{i} \log \left(\check{q}_{i} \circ \check{\Psi}_{i}^{-1}\right)+\check{\tau} .
$$

First of all, we can smoothly deform $f$ and $g$ through smooth functions compatible with $\left(V_{i}\right)_{i \in S}$ by changing $q_{i}, \check{q}_{i}, b_{i}, \breve{b}_{i}$ and $\tau$ and $\check{\tau}$ so that $q_{i}=\check{q}_{i}, b_{i}=\check{b}_{i}$ and $\tau=\check{\tau}=0$. Hence we can assume that

$$
f=\sum_{i \in S-\star_{S}} b_{i} \log \left(q_{i} \circ \Psi_{i}^{-1}\right) \text { and } g=\sum_{i \in S-\star_{S}} b_{i} \log \left(q_{i} \circ \check{\Psi}_{i}^{-1}\right) .
$$

Lemma 5.17 tells us that there is a smooth family of regulations $\left(\Psi_{i}^{t}\right)_{i \in S-\star_{S}}, t \in[0,1]$ compatible with $V_{\star_{S}}$ connecting $\left(\Psi_{i}\right)_{i \in S-\star_{S}}$ and $\left(\check{\Psi}_{i}\right)_{i \in S-\star_{S}}$ and hence we get a smooth family of functions

$$
f_{t}=\sum_{i \in S-\star_{S}} b_{i} \log \left(q_{i} \circ\left(\Psi_{i}^{t}\right)^{-1}\right)
$$

compatible with $\left(V_{i}\right)_{i \in S}$ (after possibly shrinking the region on which $q_{i}$ is not equal to 1 so that it fits inside $\left.\cap_{t \in[0,1]} \operatorname{Dom}\left(\Psi_{t}\right)\right)$. This is a smooth family of functions compatible with $\left(V_{i}\right)_{i \in S}$ joining $f$ and $g$.

Lemma 5.20. Let $(X, \omega)$ be a symplectic manifold with a choice of grading and $C \subset X$ a contact hypersurface with a contact form a compatible with the contact structure satisfying $d \alpha=\left.\omega\right|_{C}$. Then $(C, \operatorname{ker}(\alpha))$ has a natural induced choice of grading.

We will call such a grading the induced grading on $C$. 
Proof. Let $X_{\alpha}$ be a smooth section of the bundle $\left.T X\right|_{C} \longrightarrow C$ equal to the $\omega$-dual of $\alpha$. Since $d \alpha=\left.\omega\right|_{C}$ and $\alpha$ is a contact form, we get that $X_{\alpha}$ is transverse to $C$. Let $R$ be the Reeb vector field of $\alpha$ and define $\xi_{C} \equiv \operatorname{ker}(\alpha)$. Let $\left.H \subset T X\right|_{C}$ be the 2-dimensional symplectic vector subbundle spanned by $X_{\alpha}$ and $R$. Then we have the following direct sum decomposition of symplectic vector bundles

$$
\left.(T M, \omega)\right|_{C} \cong\left(\xi_{C}, d \alpha\right) \oplus\left(H,\left.\omega\right|_{H}\right) .
$$

Since $X_{\alpha}, R$ is a symplectic basis for $H$ at each point of $C$, we have a natural symplectic trivialization of $\left(H,\left.\omega\right|_{H}\right)$ sending $X_{\alpha}, R$ to the standard symplectic basis vectors on $\mathbb{C}$. Hence we have a natural isomorphism:

$$
\left.(T M, \omega)\right|_{C} \cong\left(\xi_{C}, d \alpha\right) \oplus\left(\mathbb{C}, \omega_{s t d}\right) .
$$

Now choose an almost complex structure $J$ on $M$ compatible with $\omega$ so that its restriction to $\left.T M\right|_{C}$ is equal to $J_{C} \oplus i$ with respect to the splitting (5.26) where $J_{C}$ is an almost complex structure on $\xi_{C}$ compatible with $\left.d \alpha\right|_{\xi_{C}}$ and $i$ is the standard complex structure on $\mathbb{C}$.

Now we will use the natural correspondence between gradings and trivializations of the canonical bundle as stated in Appendix A. Let $\Phi: \kappa_{J} \longrightarrow X \times \mathbb{C}$ be the choice of trivialization of the canonical bundle of $(T M, J)$ associated to the grading on $(X, \omega)$ (See Definition 7.7 in Appendix A). Since $\left.J\right|_{C}=J_{C} \oplus i$ under the splitting (5.26) , we get a natural trivialization $\Phi_{C}: \kappa_{J_{C}} \longrightarrow C \times \mathbb{C}$ induced from the trivialization $\left.\Phi\right|_{C}$. The induced grading on $\left(C, \xi_{C}\right)$ is then the grading associated to the trivialization $\Phi_{C}$ as in Definition 7.7 .

We wish to use functions compatible with a resolution divisor to construct it's 'link'. The following proposition tells us how to at least start doing this.

Proposition 5.21. Let $\left(\mathcal{O}_{X}\left(\sum_{i \in S} m_{i} V_{i}\right), \Phi, \theta\right)$ be a model resolution. Define $K \equiv \cup_{i \in S-\star_{S}} V_{i}$. Let $f: X-K \longrightarrow \mathbb{R}$ be a smooth function compatible with $\left(V_{i}\right)_{i \in S}$. Then there is a smooth function $g: X-K \longrightarrow \mathbb{R}$ and an open neighborhood $U$ of $K$ so that $\left.d f\left(X_{\theta+d g}^{\omega}\right)\right|_{U}>0$ and $d f_{\star}\left(\left.X_{\theta_{\star}+d g_{\star}}^{\omega_{\star}}\right|_{U \cap V_{\star_{S}}}>0\right.$ where $\omega$ is the symplectic form associated to our model resolution, $\left.\omega_{\star} \equiv \omega\right|_{V_{\star} S},\left.f_{\star} \equiv f\right|_{V_{\star_{S}}-K}$ and $\left.g_{\star} \equiv g\right|_{V_{\star} S}-K$.

The proof of this proposition is almost exactly the same as the proof of McL16. Proposition 5.8]. The only difference is that we have to take into account the additional submanifold $V_{\star_{S}}$. For the sake of completeness we have produced the proof below. We also have a parameterized version of the proposition above.

Proposition 5.22. Let $\mathcal{M}_{t} \equiv\left(\mathcal{O}_{X}\left(\sum_{i \in S} m_{i} V_{i}\right), \Phi, \theta_{t}\right), t \in[0,1]$ be a smooth family of model resolutions. Define $K \equiv \cup_{i \in S-\star_{S}} V_{i}$. Let $f_{t}: X-K \longrightarrow \mathbb{R}, t \in[0,1]$ be a smooth family of functions compatible with $\left(V_{i}\right)_{i \in S}$. Then there is a smooth family of functions $g_{t}: X-K \longrightarrow \mathbb{R}, t \in[0,1]$ and an open neighborhood $U$ of $K$ so that $\left.d f_{t}\left(X_{\theta+d g_{t}}^{\omega_{t}}\right)\right|_{U}>0$ and $\left.d f_{\star, t}\left(X_{\theta_{\star}+d g_{\star, t}}^{\omega_{\star, t}}\right)\right|_{U \cap V_{\star_{S}}}>0$ where $\omega_{t}$ is the symplectic form associated to $\mathcal{M}_{t},\left.\omega_{\star, t} \equiv \omega_{t}\right|_{V_{\star} S}$, $\left.f_{\star, t} \equiv f_{t}\right|_{V_{\star}-K}$ and $\left.g_{\star, t} \equiv g_{t}\right|_{V_{\star_{S}}-K}$ for all $t \in[0,1]$.

The proof of this proposition is almost exactly the same as the proof of Proposition 5.21 except that all variables are now parameterized by $t$. Therefore for notational simplicity we will just prove Proposition 5.21. Before we prove this we need a few preliminary technical lemmas. The following lemma is very similar to [McL16, Lemma 5.12]. This lemma should be thought of as a local version of Proposition 5.21. 
Lemma 5.23. Let $\left(\mathcal{O}_{X}\left(\sum_{i \in S} m_{i} V_{i}\right), \Phi, \theta\right)$ be a model resolution and fix a metric $\|\cdot\|$ on $X$. Define $K \equiv \cup_{i \in S-\star_{S}} V_{i}$. Fix $I \subset S$ and let $\omega$ be the symplectic form associated to our model resolution. Let $U \subset K$ be an open set with the property that $\bar{U} \cap V_{I^{\prime}}$ is contained inside a contractible Darboux chart of $V_{I^{\prime}}$ for all $I^{\prime} \subset S$ and so that $\bar{U} \cap V_{i}=\emptyset$ for all $i \in S-I$.

Then there is a smooth function $g: X-K \longrightarrow \mathbb{R}$ so that for any function $f: X-K \longrightarrow \mathbb{R}$ compatible with $\left(V_{i}\right)_{i \in S}$, we have

(1) $\left.d f\left(X_{\theta+d g}^{\omega}\right)\right|_{W_{f}}>c_{f}\|\theta+d g\|\|d f\|_{W_{f}}$ for some constant $c_{f}>0$ and some small neighborhood $W_{f}$ of $\bar{U} \cap V_{I}$,

(2) $\left.d f_{\star}\left(X_{\theta_{\star}+d g_{\star}}^{\omega_{\star}}\right)\right|_{W_{f, \star}}>\left.c_{f}\left\|\theta_{\star}+d g_{p, \star}\right\|\left\|d f_{\star}\right\|\right|_{W_{f, \star}}$ where $\left.f_{\star} \equiv f\right|_{V_{\star} S}-K, W_{f, \star} \equiv W_{f, \star} \cap V_{\star S}$, $\left.\theta_{\star} \equiv \theta\right|_{V_{\star}-K}$ and $\left.g_{\star} \equiv g\right|_{V_{\star}-K}$ and

(3) $a_{1}\|d f\|<\|\theta+d g\|<a_{2}\|d f\|$ inside $W_{f}$ for some constants $a_{1}, a_{2}>0$.

Proof. Define $\widehat{I} \equiv I-\star_{S}$ and let $n$ be the dimension of $X$ divided by 2 . Since $\bar{U} \cap V_{i}$ is contained inside a contractible Darboux chart we have, by a Moser argument, symplectic coordinates $x_{1}^{i}, y_{1}^{i}, \cdots, x_{n}^{i}, y_{n}^{i}$ defined on some neighborhood $W_{i}$ of $\bar{U} \cap V_{i}$ in $X$ so that $V_{i} \cap W_{i}=\left\{x_{1}^{i}=\right.$ $\left.y_{1}^{i}=0\right\}$ for each $i \in \widehat{I}$. We can also choose $W_{i}$ so that $W_{i} \cap V_{j}=\emptyset$ for all $j \in S-I$ and so that $\bar{W}_{i}$ is a compact contractible codimension 0 submanifold of $X$ with boundary so that $\partial \bar{W}_{i}$, $\left(V_{j}\right)_{j \in S}$ are transversely intersecting. Define $W_{i}^{\prime} \equiv W_{i}-V_{i}$ and let $P_{i}: \widetilde{W}_{i}^{\prime} \longrightarrow W_{i}^{\prime}$ be the cover corresponding to the subgroup of $\pi_{1}\left(W_{i}^{\prime}\right)$ generated by loops wrapping around $V_{i}$ near $V_{i}$ for each $i \in \widehat{I}$. In other words, the cover corresponding to the image of $\pi_{1}\left(T_{i}-V_{i}\right)$ in $\pi_{1}\left(W_{i}^{\prime}\right)$ where $T_{i}$ is a small tubular neighborhood of $V_{i} \cap W_{i}$ in $W_{i}$. Let $r_{i}: W_{i}^{\prime} \longrightarrow \mathbb{R}, \vartheta_{i}: W_{i}^{\prime} \longrightarrow \mathbb{R} / 2 \pi \mathbb{Z}$ be functions satisfying $x_{1}^{i}=r_{i} \cos \left(\vartheta_{i}\right), y_{1}^{i}=r_{i} \sin \left(\vartheta_{i}\right)$. Define $\rho_{i} \equiv \frac{1}{2}\left(r_{i}\right)^{2}, \widetilde{\rho}_{i} \equiv P_{i}^{*} \rho_{i}, \widetilde{y}_{j}^{i} \equiv P_{i}^{*} y_{j}^{i}$ and $\widetilde{x}_{j}^{i} \equiv P_{i}^{*} x_{j}^{i}$ for all $i \in \widehat{I}$ and $j \in\{1, \cdots, n\}$. Let $\widetilde{\vartheta}_{i}: \widetilde{W}_{i}^{\prime} \longrightarrow \mathbb{R}$ be smooth function whose value $\bmod 2 \pi$ is equal to $P_{i}^{*} \vartheta_{i}$. Also let $\widetilde{\omega} \equiv P_{i}^{*}\left(\left.\omega\right|_{W_{i}^{\prime}}\right)$ and $\widetilde{\theta} \equiv P_{i}^{*}\left(\left.\theta\right|_{W_{i}^{\prime}}\right)$.

Then $\widetilde{\omega}=d\left(\widetilde{\rho}_{i}\right) \wedge d\left(\widetilde{\vartheta}^{i}\right)+\sum_{j=2}^{n} d\left(\widetilde{x}_{j}^{i}\right) \wedge d\left(\widetilde{y}_{j}^{i}\right)$. Therefore there is a natural symplectic embedding of $\iota_{i}: \widetilde{W}_{i}^{\prime} \hookrightarrow \mathbb{C}^{n+1}$ so that the standard symplectic coordinates $x_{1}, y_{1}, \cdots, x_{n}, y_{n}$ in $\mathbb{C}^{n}$ restricted to $\widetilde{W_{i}^{\prime}}$ are $\widetilde{\rho}_{i}, \widetilde{\vartheta}_{i}, \widetilde{x}_{2}^{i}, \widetilde{y}_{2}^{i}, \cdots, \widetilde{x}_{n}^{i}, \widetilde{y}_{n}^{i}$ respectively. Let $\widehat{W}_{i}^{\prime} \subset \mathbb{C}^{n+1}$ be the closure of $P_{i}^{-1}\left(W_{i}^{\prime}\right)$ inside $\mathbb{C}^{n+1}$ and let $\widehat{V}_{I-i}^{\prime}$ be the closure of $P_{i}^{-1}\left(V_{I-i}\right)$ inside $\mathbb{C}^{n+1}$. Then $\widehat{W}_{i}^{\prime}$ is a codimension 0 submanifold of $\mathbb{C}^{n+1}$ with boundary and corners and $\widehat{V}_{I-i}^{\prime}$ is a codimension $2(|I|-1)$ submanifold of $\widehat{W}_{i}^{\prime}$ with boundary and corners where one part of the boundary is $\check{V}_{I-i} \equiv\left\{x_{1}=0\right\} \cap \widehat{V}_{I-i}^{\prime}$. Let $\bar{W}_{i}$ be the closure of $W_{i}$ in $X$. The map $P_{i}$ extends to a map $\bar{P}_{i}: \widehat{W}_{i}^{\prime} \longrightarrow \bar{W}_{i}$ whose fibers over $\bar{W}_{i} \cap V_{i}$ are one dimensional. Also $\check{V}_{I-i}$ is equal to $\bar{P}_{i}^{-1}\left(V_{I}\right)$ and $\widehat{V}_{I-i}^{\prime}=\bar{P}_{i}^{-1}\left(V_{I-i}\right)$. 


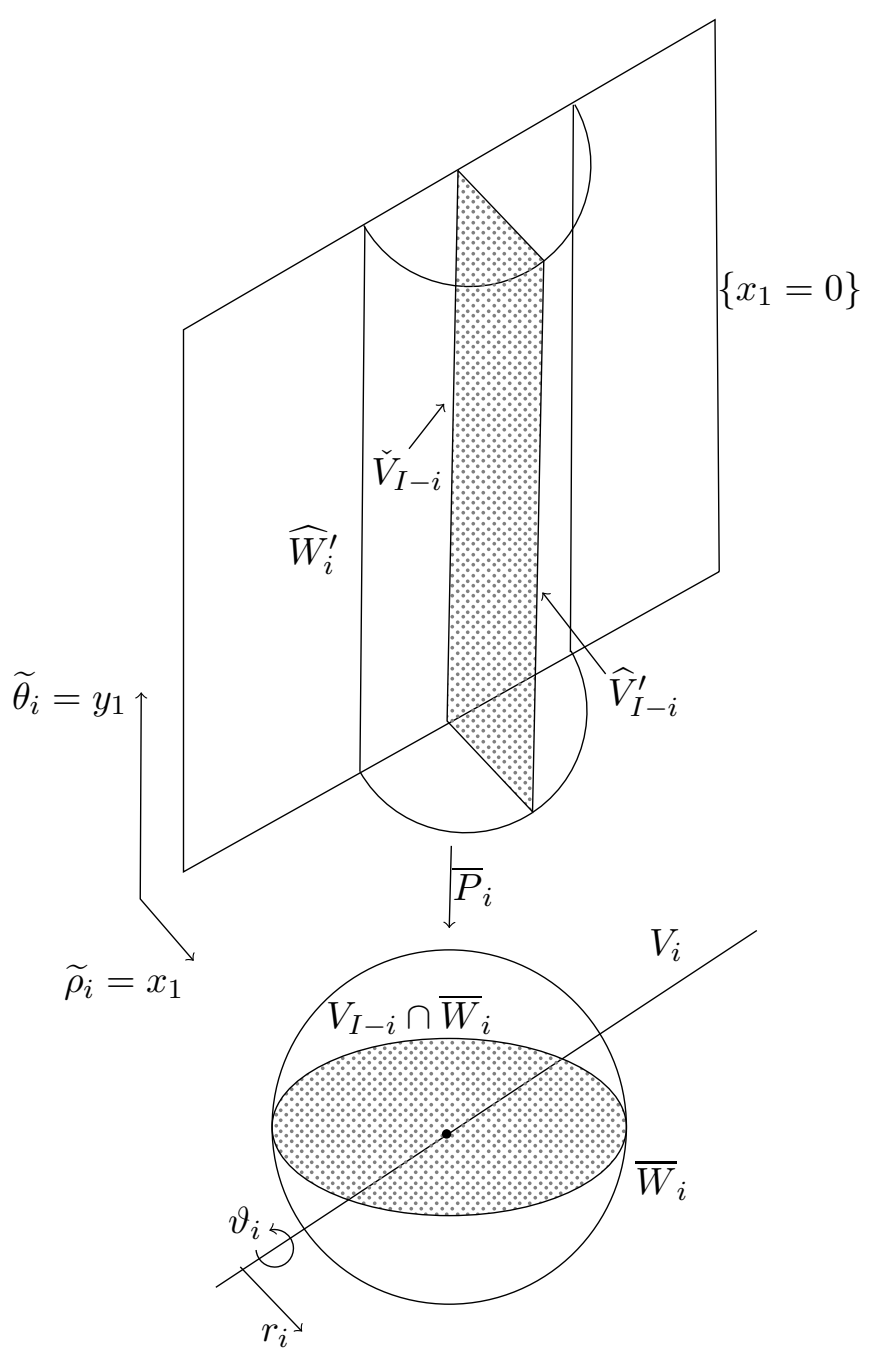

Let $w_{i}>0$ be the wrapping number of $\theta$ around $V_{i}$. Let $\left.H \subset T \mathbb{C}^{n+1}\right|_{\check{V}_{I-i}}$ be a 2 dimensional symplectic subbundle over $\check{V}_{I-i}$ containing ker $\left.D \bar{P}_{i}\right|_{\check{V}_{I-i}}=\left.\operatorname{Span}\left(\frac{\partial}{\partial y_{1}}\right)\right|_{\check{V}_{I-i}}$ so that $H$ is contained in $T \widehat{V}_{I-i}^{\prime}$. Let $T^{\perp} \widehat{V}_{I-i}^{\prime}$ be the set of vectors which are symplectically orthogonal to $T \widehat{V}_{I-i}^{\prime}$ and define $\left.\widehat{H} \equiv H \oplus T^{\perp} \widehat{V}_{I-i}^{\prime}\right|_{\check{V}_{I-i}}$.

Choose a smooth function $\widetilde{g}_{i}: \mathbb{C}^{n+1} \longrightarrow \mathbb{R}$ so that:

(a) $\widetilde{g}_{i}\left(x_{1}, y_{1}, \cdots, x_{n}, y_{n}\right)=\widetilde{g}_{i}\left(x_{1}, y_{1}+2 \pi, x_{2}, y_{2}, \cdots, x_{n}, y_{n}\right)+w_{i}$,

(b) $d x_{1}\left(X_{d \widetilde{g}_{i}}\right)>0$ at each point of $\check{V}_{I-i}$ and

(c) the $\left.\omega_{\mathbb{C}^{n+1}}\right|_{\widehat{H}^{-}}$dual of $\left.d \widetilde{g}_{i}\right|_{\widehat{H}}$ is tangent to $\widehat{V}_{I-i}^{\prime}$ at each point of $\check{V}_{I-i}$ where $\omega_{\mathbb{C}^{n+1}}$ is the standard symplectic structure on $\mathbb{C}^{n+1}$.

Condition (c) implies that $X_{d \widetilde{g}_{i}}$ is tangent to $\widehat{V}_{I-i}^{\prime}$ at each point of $\check{V}_{I-i}$. By (a) there is a closed 1-form $\beta_{i} \in \Omega^{1}\left(W_{i}^{\prime}\right)$ whose pullback to $\widetilde{W}_{i}^{\prime}$ is equal to $\left.d \widetilde{g}_{i}\right|_{W_{i}^{\prime}}$.

Define $W^{\prime} \equiv \cap_{i \in \hat{I}} W_{i}$. Let $\theta_{1} \in \Omega^{1}\left(W^{\prime}\right)$ be any 1 -form of bounded norm satisfying $d \theta_{1}=$ $\left.\omega\right|_{W^{\prime}}$. Define

$$
\Theta \in \Omega^{1}\left(W^{\prime}-K\right), \quad \Theta \equiv \theta_{1}+\sum_{i \in \widehat{I}} \beta_{i} .
$$


By (a), we get that the wrapping number of $\Theta$ around $V_{i} \cap W^{\prime}$ is $w_{i}$ for all $i \in \widehat{I}$. Hence (after shrinking $\left(W_{i}\right)_{i \in \widehat{I}}$ so that $W^{\prime}$ deformation retracts on to $\left.W^{\prime} \cap K\right)$ there is a function $g: X-K \longrightarrow \mathbb{R}$ so that $\left.(\theta+d g)\right|_{W^{\prime}}=\left.\Theta\right|_{W^{\prime}}$.

Let $f: X-K \longrightarrow \mathbb{R}$ be compatible with $\left(V_{i}\right)_{i \in S}$ and let $\left(\Psi_{i}\right)_{i \in S-\star_{S}}$ be its associated regularizations. Then by definition $\left.f\right|_{W^{\prime}}=\sum_{i \in \widehat{I}} b_{i} \log \left(q_{i} \circ \Psi_{i}^{-1}\right)+\tau$ where

- $\tau: W^{\prime} \longrightarrow \mathbb{R}$ is a smooth function and $b_{i}>0$ are constants for all $i \in \widehat{I}$,

- $q_{i}: \operatorname{Dom}\left(\Psi_{i}\right) \longrightarrow \mathbb{R}$ is equal to a square norm near $V_{i}$, equal to 1 outside a compact subset of $\operatorname{Dom}\left(\Psi_{i}\right)$ and non-zero on $\operatorname{Dom}\left(\Psi_{i}\right)-V_{i}$ and

- $\log \left(q_{i} \circ \Psi_{i}^{-1}\right)$ is defined to be zero outside $\operatorname{Im}\left(\Psi_{i}\right)$.

Define $s_{i} \equiv \log \left(q_{i} \circ \Psi_{i}^{-1}\right)$ and $\left.s_{i, \star} \equiv s_{i}\right|_{V_{\star}-K}$. Define $\left.\beta_{i, \star} \equiv \beta_{i}\right|_{V_{\star} \cap\left(W^{\prime}-K\right)}$. Since

$$
c_{1} / r_{i}<\left\|\beta_{i}\right\|<c_{2} / r_{i}, \quad c_{1} / r_{i}<\left\|d s_{i}\right\|<c_{2} / r_{i}
$$

for some $c_{1}, c_{2}>0$ and $\left\|\theta_{1}\right\|$ is bounded, part (3) of our lemma holds.

Since $\|\tau\|$ and $\left\|\theta_{1}\right\|$ are bounded and since Equation (5.27) holds, it is sufficient for us to prove the following statements:

(1) $d s_{i}\left(\sum_{j \in \widehat{I}} X_{\beta_{i}}\right)>\frac{c_{f}}{\left(r_{i}\right)^{2}}$ inside some small neighborhood $W_{f}$ of $W^{\prime} \cap V_{I}$ and some constant $c_{f}>0$ for all $i \in \widehat{I}$,

(2) $d s_{i, \star}\left(\sum_{j \in \widehat{I}} X_{\beta_{j, \star}}\right)>\frac{c_{f}}{\left(r_{i}\right)^{2}}$ inside $W_{f} \cap V_{\star_{S}}$ for all $i \in \widehat{I}$.

Let $\widehat{V}_{\star_{S}}$ be closure of $P_{i}^{-1}\left(V_{\star_{S}}\right)$ inside $\mathbb{C}^{n+1}$. Let $|\cdot|$ be the standard norm on $\mathbb{C}^{n+1}$. Since there is diffeomorphism $\Phi_{i}: W_{i} \longrightarrow W_{i}$ which is the identity on $V_{i} \cap W_{i}$, fixing $V_{j} \cap W_{i}$ for all $j \in I-i$ that is also isotopic to the identity through such diffeomorphisms and pulling back $s_{i}$ to $\log \left(\rho_{i}\right)$, we have that inequalities (1) and (2) above can be deduced from:

(1) $d \widetilde{\rho}_{i}\left(D \widetilde{\Phi}_{i}\left(X_{d \widetilde{g}_{i}}\right)\right)>c_{f}^{\prime}$ inside $\widetilde{W}_{f}-\bar{P}_{i}^{-1}\left(V_{i}\right)$ for some small neighborhood $\widetilde{W}_{f}$ of $\check{V}_{I-i}$ and some constant $c_{f}^{\prime}>0$ where $\widetilde{\Phi}_{i}: \widetilde{W}_{i}^{\prime} \longrightarrow \widetilde{W}_{i}^{\prime}$ is a lift of $\left.\Phi\right|_{W_{i}^{\prime}}: W_{i}^{\prime} \longrightarrow W_{i}^{\prime}$,

(2) $d \widetilde{\rho}_{i}\left(D \widetilde{\Phi}_{i}\left(X_{P_{i}^{*} \beta_{j}}\right)\right) \rightarrow 0$ as we approach $\check{V}_{I-i}$ for all $j \in \widehat{I}-i$ and

(3) $X_{d \widetilde{g}_{i}}$ is tangent to $\widehat{V}_{\star_{S}}$ along $\check{V}_{I-i}$ for all $i \in \widehat{I}$.

These properties follow from (a) (c) above.

Lemma 5.24. Let $\left(\mathcal{O}_{X}\left(\sum_{i \in S} m_{i} V_{i}\right), \Phi, \theta\right)$ be a model resolution and fix a metric $\|\cdot\|$ on $X$. Define $K \equiv \cup_{i \in S-\star_{S}} V_{i}$ and let $f: X-K \longrightarrow \mathbb{R}$ be a smooth function compatible with $\left(V_{i}\right)_{i \in S}$. Then there is a smooth function $h: X-K \longrightarrow \mathbb{R}$ and constants $a_{1}, a_{2}>0$ so that $a_{1}\|d f\|<\|\theta+d h\|<a_{2}\|d f\|$ near $K$.

Proof. We will use part (3) of Lemma 5.23 and an induction argument to do this. Choose open sets $U_{1}, \cdots, U_{m}$ in $X$ along with subsets $I_{1}, \cdots I_{m} \subset S$ so that

- $\cup_{j=1}^{m}\left(U_{j} \cap V_{I_{j}}\right)=K$,

- $\bar{U}_{j} \cap V_{I^{\prime}}$ is contained inside a contractible Darboux chart of $V_{I^{\prime}}$ for all $I^{\prime} \subset S, j \in$ $\{1, \cdots, m\}$ and

- so that $\bar{U}_{j} \cap V_{k}=\emptyset$ for all $j \in S-I_{j}$ and all $j \in\{1, \cdots, m\}$.

Let $\check{U}_{1}, \cdots, \check{U}_{m}$ be open sets of $X$ so that the closure of $\check{U}_{i}$ is contained inside $U_{i}$ for all $i \in\{1, \cdots, m\}$ and $\cup_{j=1}^{m}\left(\check{U}_{j} \cap V_{I_{j}}\right)=K$. Define $U_{<k} \equiv \cup_{j<k} U_{j}$ and $\check{U}_{<k} \equiv \cup_{j<k} \check{U}_{j}$.

Suppose, by induction, there is a smooth function $h_{\prec}: X-K \longrightarrow \mathbb{R}$ and constants $a_{1}^{\prec}, a_{2}^{\prec}>0$ so that

$$
a_{1}^{\prec}\|d f\|<\left\|\theta+d h_{\prec}\right\|<a_{2}^{\prec}\|d f\|
$$


on a neighborhood $N \subset U_{<k}$ of the closure of $\check{U}_{<k} \cap K$ for some $k \in\{1, \cdots, m\}$. By Lemma 5.23, there is a function $h_{=}: X-K \longrightarrow \mathbb{R}$ and constants $a_{1}^{\bar{y}}, a_{2}^{\overline{ }}>0$ so that

$$
a_{1}^{=}\|d f\|<\left\|\theta+d h_{\prec}+d h_{=}\right\|<a_{2}^{=}\|d f\|
$$

on a neighborhood $W_{k}$ of $U_{k} \cap K$ in $X$. Now let $\rho: X \longrightarrow[0,1]$ be a smooth function equal to 0 on a neighborhood of $\check{U}_{<k} \cap K$ and which is 1 outside a compact subset of $N$. Define $h_{\preceq} \equiv h_{\prec}+\rho h_{=}$. Equations (5.28) and (5.29) tell us that $\left\|d h_{=}\right\| \leq\left(a_{2}^{\prec}+a_{2}^{\bar{y}}\right)\|d f\|$ near $U_{k} \cap\left(N-\check{U}_{<k}\right) \cap K$ which implies that $\left|h_{=}\right|<C|f|+\check{C}$ near $\left(N-\check{U}_{<k}\right) \cap K$ for some $C, \check{C}>0$. Hence

$$
a_{1}^{\preceq}\|d f\|<\left\|\theta+d h_{\preceq}\right\|<a_{2}^{\preceq}\|d f\|
$$

near $\overline{\check{U}_{<k+1}} \cap K$ for some $a_{1}^{\preceq}, a_{2}^{\preceq}>0$ and so we are finished by induction.

Proof of Proposition 5.21. By Lemma 5.24 we can add an exact 1-form to $\theta$ so that

$$
b_{1}\|d f\|<\|\theta\|<b_{2}\|d f\|
$$

inside a neighborhood $N$ of $K$ for some constants $b_{1}, b_{2}>0$. By Lemma 5.23 we can find open sets $W_{1}, \cdots, W_{m}$ of $X$ covering $K$, smooth functions $g_{i}: X-K \longrightarrow \mathbb{R}, i=1, \cdots, m$, and a constant $c>0$ so that

(1) $\left.d f\left(X_{\theta+d g_{i}}^{\omega}\right)\right|_{W_{i}}>c\left\|\theta+d g_{i}\right\|\|d f\|_{W_{i}}$

(2) $\left.d f_{\star}\left(X_{\theta_{\star}+d g_{i}}^{\omega_{\star}}\right)\right|_{W_{\star}}>\left.c\left\|\theta+d g_{i, \star}\right\|\left\|d f_{\star}\right\|\right|_{W_{i, \star}}$ where $\left.f_{\star} \equiv f\right|_{V_{\star}-K}, W_{i, \star}=W_{i} \cap V_{\star_{S}}$, $\theta_{\star}=\left.\theta\right|_{V_{\star}-K}$ and $g_{i, \star}=\left.g_{i}\right|_{V_{\star_{S}}-K}$ and

(3) $c\|d f\|<\left\|\theta+d g_{i}\right\|<\check{c}\|d f\|$ inside $W_{i}$ for some constants $c, \check{c}>0$.

Now choose smooth functions $\rho_{i}, i=1, \cdots, m$ so that $\left.\sum_{i=1}^{m} \rho_{i}\right|_{K}=1$ and $\rho_{i}=0$ outside a compact subset of $W_{i}$ for each $i=1, \cdots, m$. We define

$$
g: X-K \longrightarrow \mathbb{R}, \quad g \equiv \sum_{i=1}^{m} \rho_{i} g_{i} .
$$

We define $\left.g_{\star} \equiv g\right|_{V_{\star_{S}}-K}$. The inequality (5.30) combined with property (3) above tells us that $\left|g_{i}\right|<C|f|+\check{C}$ for some $C, \check{C}>0$ near $W_{i} \cap K$. This means that $d f\left(X_{\theta+d g}^{\omega}\right)>c\|\theta+d g\|\|d f\|$ near $K, d f\left(X_{\theta_{\star}+d g_{\star}}^{\omega}\right)>c\left\|\theta_{\star}+d g_{\star}\right\|\|d f\|$ near $V_{\star_{S}} \cap K$ and $a_{1}\|d f\|<\|\theta+d g\|<a_{2}\|d f\|$ near $K$ for some constants $a_{1}, a_{2}>0$.

Definition 5.25. The link of a model resolution $\left(\mathcal{O}_{X}\left(\sum_{i \in S} m_{i} V_{i}\right), \Phi, \theta\right)$ is a contact pair with normal bundle data $\left(B \subset C, \xi_{C}, \Phi_{B}\right)$ defined as follows: By [MTZ14b, Lemma 4.1], there is a tuple of regularizations $\left(\Psi_{i}\right)_{i \in S-\star_{S}}$ compatible with $V_{\star_{S}}$ as in Definition 5.15, Define $K \equiv \cup_{i \in S-\star_{S}} V_{i}$. Let $f: X-K \longrightarrow \mathbb{R}$ be a smooth function compatible with $\left(V_{i}\right)_{i \in S}$ so that $\left(\Psi_{i}\right)_{i \in S-\star_{S}}$ are associated regularizations of $f$. Then $\left.f_{\star} \equiv f\right|_{V_{\star_{S}}}$ is a smooth function compatible with $\left(V_{i} \cap V_{\star_{S}}\right)_{i \in S-\star_{S}}$. Hence by Proposition 5.21 we have that $d f\left(X_{\theta+d g}\right)>0$ and $d f_{\star}\left(X_{\theta_{\star}+d g_{\star}}\right)>0$ in an open neighborhood $U$ of $K$ for some smooth function $g: X-K \longrightarrow \mathbb{R}$ where $\left.g_{\star} \equiv g\right|_{V_{\star} S}-K$ and $\left.\theta_{\star} \equiv \theta\right|_{V_{\star_{S}}-K}$. Let $c \ll-1$ be a constant satisfying $f^{-1}(c) \subset U$. Define

$$
C \equiv f^{-1}(c), \quad B \equiv C \cap V_{\star_{S}},\left.\quad \xi_{C} \equiv \operatorname{ker}(\theta+d g)\right|_{C} .
$$

Finally the trivialization $\Phi_{B}$ of the normal bundle of $B$ in $C$ is induced from the trivialization $\Phi$ since the normal bundle of $B$ is naturally isomorphic as an oriented vector bundle to $\left.\mathcal{N}_{X} V_{\star_{S}}\right|_{B}$ which in turn is naturally isomorphic to $\left.\mathcal{O}_{X}\left(\sum_{i \in S} m_{i} V_{i}\right)\right|_{B}$ since $m_{\star_{S}}=1$. 
If $\left(\mathcal{O}_{X}\left(\sum_{i \in S} m_{i} V_{i}\right), \Phi, \theta\right)$ has a grading then this gives us an induced grading on the link as in the proof of Lemma 5.20 since $C-B$ is a contact hypersurface of $\left(X-\cup_{i \in S-\star_{S}} V_{i}, \omega\right)$ where $\omega$ is the symplectic form associated to our model resolution. We will call this the induced grading on $\left(B \subset C, \xi_{C}, \Phi_{B}\right)$.

The link does not depend on the choice of neighborhood $U$, constant $C$ or function $f$ by the following Lemma:

Lemma 5.26. Suppose that $\left(\mathcal{O}_{X}\left(\sum_{i \in S} m_{i} V_{i}\right), \Phi, \theta\right)$ and $\left(\mathcal{O}_{\widehat{X}}\left(\sum_{i \in \widehat{S}} \widehat{m}_{i} \widehat{V}_{i}\right), \widehat{\Phi}, \widehat{\theta}\right)$ are (graded) isotopic. Then their links are also (graded) isotopic for any choice of neighborhood $U$ constant $C$ or function $f$ chosen for each of these two model resolutions.

Proof. This follows from Lemma 5.19 and Proposition 5.22 ,

5.4. Constructing a Contact Open Book from a Model Resolution. The aim of this section is to construct a contact open book for each model resolution so that the contact pair associated to this open book is the link of our model resolution.

Definition 5.27. Let $\left(\mathcal{O}_{X}\left(\sum_{i \in S} m_{i} V_{i}\right), \Phi, \theta\right)$ be a model resolution with associated symplectic structure $\omega$. Suppose $\left(V_{i}\right)_{i \in S}$ admits an $\omega$-regularization

$$
\mathcal{R} \equiv\left(\left(\rho_{i}\right)_{i \in S},\left(\Psi_{I}\right)_{I \subset S}\right)
$$

with associated Hermitian structures $\left(\rho_{I ; i}, \nabla^{(I ; i)}\right)$ on $\left.\mathcal{N}_{X} V_{i}\right|_{V_{I}}$ for each $i \in I \subset S$. Let $\alpha_{I ; i} \equiv \alpha_{\rho_{I ; i}, \nabla^{(I ; i)}} \in \Omega^{1}\left(\left.\mathcal{N}_{X} V_{i}\right|_{V_{I}}-V_{I}\right)$ be the associated Hermitian connection 1-form on $\left.\mathcal{N}_{X} V_{i}\right|_{V_{I}}$. Define $K \equiv \cup_{i \in S-\star_{S}} V_{i}$. Let $w_{i}$ be the wrapping number of $\theta$ around $V_{i}$ for each $i \in S-\star_{S}$ and define $a_{\star_{S}} \equiv 0$. Let

$$
\operatorname{pr}_{I ; i}:\left.\mathcal{N}_{X} V_{I} \longrightarrow \mathcal{N}_{X} V_{i}\right|_{V_{I}}
$$

be the natural projection map for all $I \subset S$. We say that $\theta$ is compatible with $\mathcal{R}$ if the restriction of

$$
\left(\Psi_{I}\right)^{*} \theta-\sum_{i \in I} p r_{I ; i}^{*}\left(\left(\rho_{I ; i}+\frac{w_{i}}{2 \pi}\right) \alpha_{I ; i}\right)
$$

to each fiber of $\left.\pi_{\mathcal{N}_{X} V_{I}}\right|_{\Psi_{I}^{-1}(X-K)}$ is 0 for every $I \subset S$.

Lemma 5.28. Let $\left(\mathcal{O}_{X}\left(\sum_{i \in S} m_{i} V_{i}\right), \Phi, \theta\right)$ be a model resolution so that $\left(V_{i}\right)_{i \in S}$ admits an $\omega$ regularization $\mathcal{R}$. Define $K \equiv \cup_{i \in S-\star_{S}} V_{i}$. Then there is a smooth function $g: X-K \longrightarrow \mathbb{R}$ so that $\theta+d g$ is compatible with $\check{\mathcal{R}}$ for some regularization $\check{\mathcal{R}}$ which is germ equivalent to $\mathcal{R}$.

Proof. This is done by induction on the strata of $\cup_{i} V_{i}$. We will use the notation from Definition 5.27 above. Let $\preceq$ be a total order on the set of subsets of $S$ with the property that if $\left|I^{\prime}\right|<|I|$ then $I \preceq I^{\prime}$. We write $I \prec I^{\prime}$ when $I \preceq I^{\prime}$ and $I \neq I^{\prime}$. Suppose for some $I^{*} \subset S$, we have constructed open sets $U_{I}^{\prec}$ inside $\operatorname{Dom}\left(\Psi_{I}\right)$ containing $V_{I}$ for all $I \prec I^{*}$ and a smooth function $g^{\prec}: X-K \longrightarrow \mathbb{R}$ with the property that

$$
\left(\Psi_{I}\right)^{*}\left(\theta+d g^{\prec}\right)-\sum_{i \in I} p r_{I ; i}^{*}\left(\left(\rho_{I ; i}+\frac{w_{i}}{2 \pi}\right) \alpha_{I ; i}\right)
$$

vanishes along each fiber of $\left.\pi_{\mathcal{N}_{X} V_{I}}\right|_{U_{I}^{\prec} \cap \operatorname{Dom}\left(\Psi_{I}\right)}$ for all $I \prec I^{*}$.

We now want these properties to hold for all $I \preceq I^{*}$. For all $I \preceq I^{*}$, let $U_{I}^{\preceq} \subset \operatorname{Dom}\left(\Psi_{I}\right)$ be open sets containing $V_{I}$ whose closure is compact so that the closure of $U_{\bar{I}}^{\prec}$ is contained in $U_{I}^{\prec}$ when $I \prec I^{*}$ and so that $\left.\pi_{\mathcal{N}_{X} V_{I}}\right|_{U_{I}^{\prec}}$ has contractible fibers for all $I \preceq I^{*}$. Since the wrapping 
number of $\theta$ around $V_{i}$ is $w_{i}$ for all $i \in I$ and since $\left(\Psi_{I}\right)^{*}(d \theta)=\left.\omega_{\left(\rho_{I ; i}, \nabla^{(I ; i)}\right)_{i \in I}}\right|_{\operatorname{Dom}\left(\Psi_{I}\right)}$, we get that the restriction of

$$
A \equiv\left(\Psi_{I^{*}}\right)^{*}\left(\theta+d g^{\prec}\right)-\sum_{i \in I^{*}} p r_{I^{*} ; i}^{*}\left(\left(\rho_{I^{*} ; i}+\frac{w_{i}}{2 \pi}\right) \alpha_{I^{*} ; i}\right)
$$

to each fiber of $\left.\pi_{\mathcal{N}_{X} V_{I}}\right|_{\operatorname{Dom}\left(\Psi_{I^{*}}\right)}$ is exact. Also by our induction hypothesis, the restriction of $A$ to the fibers of $\left.\pi_{\mathcal{N}_{X} V_{I^{*}}}\right|_{\left(\Psi_{I^{*}}\right)^{-1}\left(\Psi_{I}\left(U_{I}^{\prec}\right)\right)}$ is 0 for all $I \subsetneq I^{*}$. This means that there is a smooth function $g=: X-K \longrightarrow \mathbb{R}$ so that $g^{=}$restricted to a small neighborhood of the closure of $\Psi_{I}\left(U_{\bar{I}}^{\prec}\right)$ is 0 for all $I \subsetneq I^{*}$ and so that $A+\left(\Psi_{I^{*}}\right)^{*} d g^{=}$restricted to each fiber of $\left.\pi_{\mathcal{N}_{X} V_{I^{*}}}\right|_{U_{I^{*}}^{\prec} \cap \operatorname{Dom}\left(\Psi_{I^{*}}\right)}$ is 0. Define $g^{\preceq} \equiv g^{\prec}+g^{=}$. Then

$$
\left(\Psi_{I}\right)^{*}\left(\theta+d g^{\preceq}\right)-\sum_{i \in I} \operatorname{pr}_{I ; i}^{*}\left(\left(\rho_{I ; i}+\frac{w_{i}}{2 \pi}\right) \alpha_{I ; i}\right)
$$

vanishes along each fiber of $\left.\pi_{\mathfrak{N}_{X} V_{I}}\right|_{U_{I}^{\preceq} \cap \operatorname{Dom}\left(\Psi_{I}\right)}$ for all $I \preceq I^{*}$. Hence by induction we have shown that there is a smooth function $g: X-K \longrightarrow \mathbb{R}$ and open subsets $U_{I} \subset \operatorname{Im}\left(\Psi_{I}\right)$ containing $V_{I}$ so that

$$
\left(\Psi_{I}\right)^{*}(\theta+d g)-\sum_{i \in I} \operatorname{pr}_{I ; i}^{*}\left(\left(\rho_{I ; i}+\frac{w_{i}}{2 \pi}\right) \alpha_{I ; i}\right)
$$

vanishes along each fiber of $\left.\pi_{\mathcal{N}_{X} V_{I}}\right|_{U_{I} \cap \operatorname{Dom}\left(\Psi_{I}\right)}$ for all $I \subset S$. By [MTZ14b, Lemma 5.5], we can shrink these open subsets $U_{I}$ so that $\check{\mathcal{R}} \equiv\left(\left(\left.\rho_{i}\right|_{\Psi_{I}\left(U_{I}\right)}\right)_{i \in S},\left(\left.\Psi_{I}\right|_{U_{I}}\right)_{I \subset S}\right)$ is a regularization.

Definition 5.29. Let

$$
\mathcal{M} \equiv\left(\mathcal{O}_{X}\left(\sum_{i \in S} m_{i} V_{i}\right), \Phi, \theta\right)
$$

be a model resolution with associated symplectic form $\omega$ and let $U \subset X$ be an open set. A regularization of $\mathcal{M}$ of radius $R$ along $U$ for some $R<1$ is an $\omega$-regularization $\mathcal{R}$ of $\left(V_{i}\right)_{i \in S}$ as in Equation (5.31) so that

(1) the line bundle $\mathcal{O}_{X}\left(\sum_{i \in S} m_{i} V_{i}\right)$ is also defined using the regularization maps $\left(\Psi_{i}\right)_{i \in S}$ from $\mathcal{R}$,

(2) $\Phi$ is radius $R$ compatible with $\mathcal{R}$ along $U$ as in Definition 5.9 and

(3) $\theta$ is compatible with $\mathcal{R}$.

A regularization of $\mathcal{M}$ along $U$ is a regularization of $\mathcal{M}$ of radius $R$ along $U$ for some $R<1$ smaller than the tube radius of $\mathcal{R}$ along $U$.

We wish to show that every model resolution is isotopic to one admitting a regularization as above. Before we do this we need a preliminary lemma.

Lemma 5.30. Let $X$ be a smooth manifold with a smooth family of cohomologous symplectic forms $\left(\omega_{t}\right)_{t \in[0,1]}$ and $\left(V_{i}\right)_{i \in S}$ a compact $S C$ divisor on $X$ with respect to $\omega_{t}$ for all $t \in[0,1]$. Define $K \equiv \cup_{i \in S} V_{i}$ and let $\theta$ be a 1 -form on $X-K$ satisfying $d \theta=\left.\omega_{0}\right|_{X-K}$. Then there exists a smooth family of 1 -forms $\left(\theta_{t}\right)_{t \in[0,1]}$ on $X-K$ so that $d \theta_{t}=\left.\omega_{t}\right|_{X-K}$ for all $t \in[0,1]$ and so that the wrapping number of $\theta_{t}$ around $V_{j}$ does not depend on $t \in[0,1]$ for all $j \in S$.

Proof. Since $\omega_{t}-\omega_{0}$ is exact for all $t \in[0,1]$, there is (by exploiting the Hodge decomposition theorem for differential forms) a smooth family of 1 -forms $\left(\beta_{t}\right)_{t \in[0,1]}$ on $X$ so that $d \beta_{t}=$ $\omega_{t}-\omega_{0}$. Let $U \subset X$ be a neighborhood of $K$ whose closure is a compact manifold with boundary which deformation retracts onto $K$ and $\rho: X \longrightarrow[0,1]$ a smooth function equal to 0 near $K$ and equal to 1 outside a compact subset of $U$. Define $\check{\beta}_{t} \equiv \theta+\beta_{t} \in \Omega^{1}(X-K)$ 
for all $t \in[0,1]$. Since $\rho \check{\beta}_{t}=0$ near $K$, we can think of this as a smooth 1 -form on $X$ by defining it to be 0 along $K$. Let $c_{t} \equiv\left[\omega_{t}-d\left(\rho \check{\beta}_{t}\right)\right] \in H^{2}(X, X-U ; \mathbb{R})$ for all $t \in[0,1]$ and define $c \equiv\left[\omega_{0}-d(\rho \theta)\right] \in H^{2}(X, X-U ; \mathbb{R})$. Since $H^{2}(X, X-U ; \mathbb{R})=H^{2}(X, X-K ; \mathbb{R})$ we have the long exact sequence:

$$
H^{1}(X-K ; \mathbb{R}) \stackrel{\alpha}{\longrightarrow} H^{2}(X, X-U ; \mathbb{R}) \stackrel{\check{\alpha}}{\longrightarrow} H^{2}(X ; \mathbb{R}) \longrightarrow H^{2}(X-K ; \mathbb{R}) .
$$

Since $\check{\alpha}\left(c_{t}\right)=\check{\alpha}(c)=\left[\omega_{0}\right]$ for all $t \in[0,1]$, we have a smooth family closed 1-forms $b_{t} \in \Omega^{1}(X-$ $K)$ so that $\alpha\left(b_{t}\right)=c-c_{t}$ for all $t \in[0,1]$. Let $\theta_{t}=\check{\beta}_{t}+b_{t}$. Then $\left[\left.\omega_{t}\right|_{U}-d\left(\rho \theta_{t}\right)\right] \in H_{c}^{2}(U ; \mathbb{R})$ is independent of $t$ which proves our lemma.

Lemma 5.31. Let $\left(\mathcal{O}_{X}\left(\sum_{i \in S} m_{i} V_{i}\right), \Phi, \theta\right)$ be a model resolution and let $U \subset X$ be a relatively compact open set. Then $\left(\mathcal{O}_{X}\left(\sum_{i \in S} m_{i} V_{i}\right), \Phi, \theta\right)$ is isotopic to a model resolution $\left(\mathcal{O}_{X}\left(\sum_{i \in S} m_{i} V_{i}\right), \widehat{\Phi}, \widehat{\theta}\right)$ admitting a regularization along $U$.

Proof. By [MTZ14b, Theorem 2.17], there a smooth family of cohomologous symplectic forms $\left(\omega_{t}\right)_{t \in[0,1]}$ so that $\omega_{0}=\omega$ and $\left(V_{i}\right)_{i \in S}$ admits an $\omega_{1}$-regularization

$$
\mathcal{R} \equiv\left(\left(\rho_{i}\right)_{i \in S},\left(\Psi_{I}\right)_{I \subset S}\right) .
$$

Lemma 5.30 then tells us that there is a smooth family of 1-forms $\left(\theta_{t}\right)_{t \in[0,1]}$ on $X-\cup_{i \in S-\star_{S}} V_{i}$ so that $\theta_{0}=\theta$ and $d \theta_{t}=\left.\omega\right|_{X-\cup_{i \in S-\star_{S}} V_{i}}$ for all $t \in[0,1]$ and so that the wrapping number of $\theta_{t}$ around $V_{i}$ is independent of $t$ for each $i \in S-\star_{S}$. We can assume that $\mathcal{O}_{X}\left(\sum_{i \in S} m_{i} V_{i}\right)$ is defined using the regularizations $\left(\Psi_{i}\right)_{i \in S}$ as changing the regularization needed to define a line bundle as in Equation (5.9) creates an isomorphic line bundle. Now we isotope $\Phi$ through trivializations to a trivialization $\widehat{\Phi}$ so that $\widehat{\Phi}$ is compatible with $\mathcal{R}$ along $U$ by Lemma 5.10 . By Lemma 5.28 we have, after replacing $\mathcal{R}$ with a germ equivalent regularization, that $\widehat{\theta} \equiv \theta_{1}+d g$ is compatible with $\mathcal{R}$ for some $g \in C^{\infty}\left(X-\cup_{i \in S-\star_{S}} V_{i}\right)$.

Hence $\left(\mathcal{O}_{X}\left(\sum_{i \in S} m_{i} V_{i}\right), \Phi, \theta\right)$ is isotopic to $\left(\mathcal{O}_{X}\left(\sum_{i \in S} m_{i} V_{i}\right), \widehat{\Phi}, \theta_{1}\right)$ which in turn is isotopic to $\left(\mathcal{O}_{X}\left(\sum_{i \in S} m_{i} V_{i}\right), \widehat{\Phi}, \widehat{\theta}\right)$ which admits a regularization along $U$.

Lemma 5.32. Let $\left(\mathcal{O}_{X}\left(\sum_{i \in S} m_{i} V_{i}\right), \Phi, \theta\right)$ be a model resolution admitting a regularization along $U$ for some relatively compact open set $U \subset X$ as in Definition 5.29. Let $\Phi_{2}$ be the composition of $\Phi$ with the natural projection $X \times \mathbb{C} \rightarrow \mathbb{C}$. Define

$$
\pi_{\Phi}: U \longrightarrow \mathbb{C},\left.\quad \pi_{\Phi} \equiv \Phi_{2} \circ s_{\left(m_{i}\right)_{i \in S}}\right|_{U}
$$

where $s_{\left(m_{i}\right)_{i \in S}}$ is the canonical section of $\mathcal{O}_{X}\left(\sum_{i} m_{i} V_{i}\right)$ as defined in Equation (5.12). Then there is some $\epsilon>0$ so that $\pi_{\Phi}^{-1}(z)$ is a symplectic submanifold of $U$ for all $z \in \mathbb{D}(\epsilon)-\{0\}$. Also the restriction of $\theta$ to $\pi_{\Phi}^{-1}\left(\left\{|z|=\epsilon^{\prime}\right\}\right)$ is a contact form for all $0<\epsilon^{\prime} \leq \epsilon$.

Proof. Since $U$ is relatively compact, it is sufficient for us to show that for every $x \in U \cap$ $\left(\cup_{i \in S} V_{i}\right)$, there is a small open set $U_{x} \subset X$ containing $x$ so that $\left.\pi_{\Phi}\right|_{U_{x} \cap \pi_{\Phi}^{-1}(\mathbb{C}-0)}$ has symplectic fibers and so that $\theta$ restricted to $\pi_{\Phi}^{-1}\left(\left\{|z|=\epsilon^{\prime}\right\}\right) \cap U_{x}$ is a contact form. We will first show that the fibers are symplectic. Suppose that $I \subset S$ is the largest set satisfying $x \in V_{I}$. Let $a_{R}$ be the function defined in Definition 5.9 and let $\Pi_{\left(m_{i}\right)_{i \in S}, I}(v)$ be as in Equation (5.13). Near $x$, we have that $a_{R}\left(\rho_{i}\right)=\rho_{i}$. Therefore

$$
\pi_{\Phi}(y)=\Phi_{2}\left(\Pi_{\left(m_{i}\right)_{i \in S}, I}\left(\Psi_{I}^{-1}(y)\right)\right)
$$


for all $y \in X$ near to $x$. Since $\Psi_{I}$ is a regularization, it is sufficient for us to show that the fibers of $\Phi_{2} \circ \Pi_{\left(m_{i}\right)_{i \in S}, I}$ restricted to a small neighborhood of $x$ inside $\mathcal{N}_{X} V_{I}$ are symplectic with respect to

$$
\omega_{\left(\rho_{I ; i}, \nabla^{(I ; i)}\right)_{i \in I}} \stackrel{\sqrt[5.4]{=}}{=} \pi_{\mathcal{N}_{X} V_{I}}^{*}\left(\left.\omega\right|_{V_{I}}\right)+\frac{1}{2} \bigoplus_{i \in I} \operatorname{pr}_{I ; i}^{*}\left(d\left(\rho_{I ; i} \alpha_{I ; i}\right)\right)
$$

where $p r_{I ; i}$ is the natural projection map from Equation (5.32). Let $W_{x} \subset V_{I}$ be a small open neighborhood of $x$ that is contractible and choose unitary trivializations $T_{i}:\left.\mathcal{N}_{X} V_{i}\right|_{W_{x}} \longrightarrow$ $W_{x} \times \mathbb{C}$ for all $i \in I$. Let $z_{i}:\left.\mathcal{N}_{X} V_{I}\right|_{W_{x}} \longrightarrow \mathbb{C}$ be the composition of $T_{i} \circ p r_{I ; i}$ with the projection map to $\mathbb{C}$. Hence along $W_{x}$, Equation (5.34) becomes:

$$
\left.\omega_{\left(\rho_{I ; i}, \nabla^{(I ; i)}\right)_{i \in I}}\right|_{W_{x}}=\pi_{\mathcal{N}_{X} V_{I}}^{*}\left(\left.\omega\right|_{V_{I}}\right)+\beta+\frac{i}{2} \bigoplus_{i \in I} d z_{i} \wedge d \bar{z}_{i}
$$

where $\beta \in \Omega^{2}\left(\left.\mathcal{N}_{X} V_{I}\right|_{W_{x}}\right)$ is a closed 2-form whose restriction to the fibers of $\left.\pi_{\mathcal{N}_{X} V_{I}}\right|_{W_{x}}$ is zero and whose restriction to the zero section is also zero. This means that near $x$ we have that $\left.\omega_{\left(\rho_{I ; i}, \nabla^{(I ; i)}\right)_{i \in I}}\right|_{W_{x}}$ is $C^{0}$ close to

$$
\check{\omega} \equiv \pi_{\mathcal{N}_{X} V_{I}}^{*}\left(\left.\omega\right|_{V_{I}}\right)+\frac{i}{2} \bigoplus_{i \in I} d z_{i} \wedge d \bar{z}_{i}
$$

We can choose our trivializations $T_{i}$ so that $\Phi_{2} \circ \prod_{\left(m_{i}\right)_{i \in S}, I}$ is equal to $\prod_{i \in I} z_{i}^{m_{i}}$ inside $\left.\pi_{\mathcal{N}_{X} V_{I}}\right|_{W_{x}}$. Since the fibers of $\prod_{i \in I} z_{i}^{m_{i}}$ are symplectic with respect to $\check{\omega}$ near $W_{x} \cap V_{I}$ and since $\omega$ is equal to $\check{\omega}$ at the point $x$, there is a small neighborhood $\widetilde{U}_{x} \subset \mathcal{N}_{X} V_{I}$ containing $x$ so that the fibers of $\left.\prod_{i \in I} z_{i}\right|_{\widetilde{U}_{x}}$ are symplectic with respect to $\left.\omega_{\left(\rho_{I ; i}, \nabla^{(I ; i)}\right)_{i \in I}}\right|_{W_{x}}$. Hence the fibers of $\left.\pi_{\Phi}\right|_{U_{x}}$ are symplectic where $U_{x} \equiv \Psi_{I}\left(\widetilde{U}_{x}\right)$.

We now wish to show that $\theta$ restricted to $Y_{r} \equiv \pi_{\Phi}^{-1}(\{|z|=r\}) \cap U_{x}$ is a contact form for all $r>0$. Since $\omega$ restricted to the fibers of $\left.\pi_{\Phi}\right|_{U_{x}}$ are symplectic, it is sufficient for us to show that $\theta$ restricted to the kernel of $\left.\omega\right|_{Y_{r}}$ is non-zero at every point for all $r>0$. By Equation (5.34) and the fact that $\Phi_{2} \circ \Pi_{\left(m_{i}\right)_{i \in S, I}}$ is equal to $\prod_{i \in I} z_{i}^{m_{i}}$ inside $\left.\mathcal{N}_{X} V_{I}\right|_{W_{x}}$, we get that the kernel of $\left.\Psi_{I}^{*} \omega\right|_{\Psi_{I}^{-1}\left(Y_{r}\right)}$ is tangent to the fibers of $\pi_{\mathcal{N}_{X} V_{I}}$ inside $\operatorname{Dom}\left(\Psi_{I}\right)$. Therefore, since the restriction of the 1-form (5.33) to the fibers of $\pi_{\mathcal{N}_{X} V_{I}}$ inside $\operatorname{Dom}\left(\Psi_{I}\right)$ is zero, $\Psi_{I}^{*} \theta$ restricted to the kernel of $\left.\Psi_{I}^{*} \omega\right|_{\widetilde{U}_{x}}$ is non-zero so long as $\widetilde{U}_{x} \subset \operatorname{Dom}\left(\Psi_{I}\right)$. Hence $\theta$ restricted to $Y_{r}$ is a contact form for all $r>0$ so long as $U_{x} \subset \operatorname{Im}\left(\Psi_{I}\right)$.

Definition 5.33. Let $\left(\mathcal{O}_{X}\left(\sum_{i \in S} m_{i} V_{i}\right), \Phi, \theta\right)$ be a model resolution admitting a radius $R$ regularization

$$
\mathcal{R} \equiv\left(\left(\rho_{i}\right)_{i \in I},\left(\Psi_{I}\right)_{I \subset S}\right)
$$

along a relatively compact open set $U \subset X$ as in Definition 5.29 where $U$ contains $K \equiv$ $\cup_{i \in S-\star_{S}} V_{i}$. Let $T_{r, I}$ be the radius $r$ tube of $V_{I}$ as in Equation (5.14). Let $\Phi_{2}: X \longrightarrow \mathbb{C}$ be the composition of $\Phi$ with the natural projection map $X \times \mathbb{C} \longrightarrow \mathbb{C}$. Choose $\epsilon>0$ small enough so that

$$
\left(\Phi_{2} \circ s_{\left(m_{i}\right)_{i \in S}}\right)^{-1}\left(\mathbb{D}_{\epsilon}\right) \cap U \subset \cup_{i \in S} T_{R, i}
$$

and so that the fibers $\left(\Phi_{2} \circ s_{\left(m_{i}\right)_{i \in S}}\right)^{-1}(z) \cap U$ are symplectic for $z \in \mathbb{D}_{\epsilon}-0$ by Lemma 5.32 , Let $\check{T}$ be a smoothing of the compact manifold with corners $\cup_{i \in S-\star_{S}} T_{R, i}$ so that

$$
\partial \check{T} \cap T_{R, \star_{S}}=\Psi_{I^{*}}\left(\pi_{\mathcal{N}_{X} V_{\star_{S}}}^{-1}\left(V_{I^{*}} \cap \partial \check{T}\right)\right) \cap T_{R, \star_{S}}
$$


$X_{\theta}$ points outwards along $\partial \check{T} \cap T_{R, \star_{S}}$ and so that $\check{T} \subset \cup_{i \in S-\star_{S}} T_{R, i}$. We also assume that the smoothing is small enough so that $\cup_{i \in S-\star_{S}} T_{3 R / 4, i}$ is contained in the interior of $\check{T}$.

Define

$$
\pi_{\Phi}: \check{T} \longrightarrow \mathbb{C},\left.\quad \pi_{\Phi} \equiv \Phi_{2} \circ s_{\left(m_{i}\right)_{i \in S}}\right|_{\check{T}}
$$

The Milnor fiber of $\left(\mathcal{O}_{X}\left(\sum_{i \in S} m_{i} V_{i}\right), \Phi, \theta\right)$ is the pair

$$
\left(M, \theta_{M}\right) \equiv\left(\pi_{\Phi}^{-1}(\epsilon),\left.\theta\right|_{\pi_{\Phi}^{-1}(\epsilon)}\right) .
$$

This is a Liouville domain for $\epsilon>0$ small enough since $X_{\theta}$ is tangent to $V_{\star_{S}}-K$ and $X_{\theta}$ is transverse to $\partial \check{T}$ and pointing outwards.

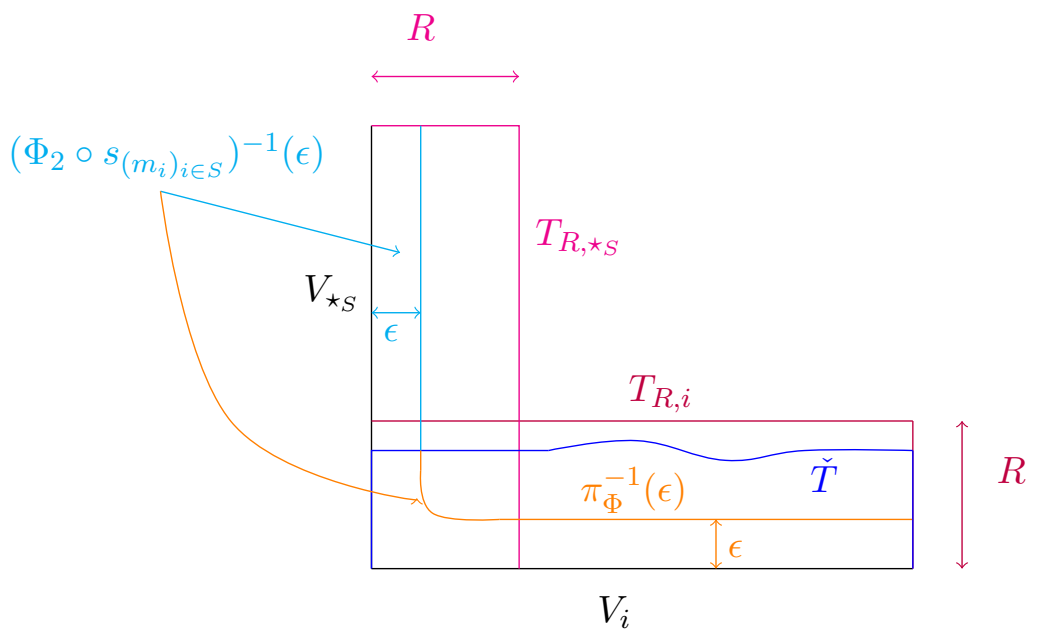

Because

- the 1-form (5.33) restricted to each fiber of $\left.\pi_{\mathcal{N}_{X} V_{I}}\right|_{\Psi_{I}^{-1}(X-K)}$ is 0 for every $I \subset S$,

- Equation (5.38) holds

- $\Phi$ is radius $R$ compatible with $\mathcal{R}$ along $U$ and

- $\cup_{i \in S-\star_{S}} T_{3 R / 4, i}$ is contained in the interior of $\check{T}$,

we get that the monodromy map $\phi: M \longrightarrow M$ of $\pi_{\Phi}$ around the loop

$$
[0,1] \longrightarrow \partial \mathbb{D}(\epsilon), \quad t \longrightarrow \epsilon e^{2 \pi i t}
$$

with respect to the symplectic connection associated to $\omega$ exists and has compact support. In addition, since $\left.\omega\right|_{\pi_{\Phi}^{-1}(\partial \mathbb{D}(\epsilon))}=\left.d \theta\right|_{\pi_{\Phi}^{-1}(\partial \mathbb{D}(\epsilon))}, \phi$ is an exact symplectomorphism with compact support. We call $\left(M, \theta_{M}, \phi\right)$ the abstract contact open book associated to $\left(\mathcal{O}_{X}\left(\sum_{i \in S} m_{i} V_{i}\right), \Phi, \theta\right)$.

Now suppose that our model resolution has a choice of grading. Since $\pi_{\Phi}^{-1}(\partial \mathbb{D}(\epsilon))$ is a contact submanifold of $(X-K, \omega)$ with contact form given by restricting $\theta$ by Lemma 5.32 after possibly shrinking $\epsilon$, we get an induced grading on this contact submanifold by Lemma 5.20. Since the contact distribution is isotopic to $Q \equiv \operatorname{ker}\left(\left.D \pi_{\Phi}\right|_{\pi_{\Phi}^{-1}(\partial(\epsilon))}\right)$ through hyperplane distributions $Q_{t}, t \in[0,1]$ where $\left.\omega\right|_{Q_{t}}$ is non-degenerate for all $t$, we get a grading

$$
\iota: \widetilde{F r}(Q) \times_{\widetilde{S p}(2 n)} \operatorname{Sp}(2 n) \cong \operatorname{Fr}(Q)
$$

on $Q$ and hence on $\left(M, d \theta_{M}\right)$. Since the parallel transport maps of $\pi_{\Phi}$ along $\partial \mathbb{D}(\epsilon)$ have lifts to $\widetilde{F r}(Q), \phi$ has an induced grading and hence $\left(M, \theta_{M}, \phi\right)$ is a graded abstract contact open book. We will call this the induced grading on $\left(M, \theta_{M}, \phi\right)$. 
Lemma 5.34. Let $\left(B_{t} \subset C, \xi_{t}, \Phi_{t}\right), t \in[0,1]$ be a smooth family of contact pairs. Then there is a smooth family of contactomorphisms $\Psi_{t}: C \longrightarrow C$ between $\left(B_{t} \subset C, \xi_{0}, \Phi_{0}\right)$ and $\left(B_{t} \subset C, \xi_{t}, \Phi_{t}\right)$ (as in Definition 3.7) for all $t \in[0,1]$ so that $\Psi_{0}=i d$.

Proof. By Gray's stability theorem, there is a smooth family of contactomorphisms $\check{\Phi}_{t}$ : $C \longrightarrow C$ starting from the identity map so that $\check{\Phi}_{t}$ is a contactomorphism between $\left(C, \xi_{0}\right)$ and $\left(C, \xi_{t}\right)$. Therefore by pulling everything back by $\check{\Phi}_{t}$, we can assume that $\xi_{t}=\xi_{0}$ for all $t \in[0,1]$. Also by Gray's stability theorem, there is a smooth family of embeddings $\iota_{t}: B_{0} \longrightarrow C$ mapping $B_{0}$ to $B_{t}$ so that

- $\left.\iota_{0}\right|_{B_{0}}: B_{0} \longrightarrow B_{0}$ is the identity map and

- $\left.\iota_{t}\right|_{B_{t}}: B_{0} \longrightarrow B_{t}$ is a contactomorphism.

Again by Gray's stability theorem, there is a neighborhood $N$ of $B$ and a smooth family of contact embeddings $\widetilde{\iota}_{t}:\left(N,\left.\xi_{0}\right|_{N}\right) \longrightarrow\left(C, \xi_{C}\right)$ whose restriction to $B_{0}$ is $\iota_{t}$ and where $\left.\widetilde{\iota}_{0}\right|_{N}: N \longrightarrow N$ is the identity map. Let $H_{t}: \widetilde{\iota}_{t}(N) \longrightarrow \mathbb{R}$ be a smooth family of functions generating the contact isotopy $\widetilde{\iota}_{t}$. By definition this means that there is a contact form $\alpha$ compatible with $\xi_{0}$ so that

$$
i_{\frac{d}{d t} \widetilde{\iota}_{t}(x)} \alpha=-H_{t}, \quad i_{\frac{d}{d t} \widetilde{\iota}_{t}(x)} d \alpha=d H_{t}-\left(i_{R} d H_{t}\right) \alpha, \quad \forall x \in \iota_{t}(N), t \in[0,1]
$$

where $R$ is the Reeb vector field of $\alpha$ (see [MS98, Lemma 3.49]). Choose a smooth family of functions $K_{t}, t \in[0,1]$ equal to $H_{t}$ near $\iota_{t}\left(B_{t}\right)$. Then $K_{t}$ generates a smooth family of contactomorphisms $\Psi_{t}$ satisfying the properties we want.

Lemma 5.35. Let $\left(\mathcal{O}_{X}\left(\sum_{i \in S} m_{i} V_{i}\right), \Phi, \theta\right)$ be a model resolution admitting a regularization

$$
\mathcal{R} \equiv\left(\rho_{i}\right)_{i \in S},\left(\Psi_{I}\right)_{I \subset S}
$$

of radius $R<1$ along an open set $U \subset X$ containing $K \equiv \cup_{i \in S-\star_{S}} V_{i}$ as in Definition 5.29. Since $m_{\star_{S}}=1$, we let

$$
\left.\Phi_{\star} \equiv \Phi\right|_{V_{\star}-K}: \mathcal{N}_{X}\left(V_{\star_{S}}-K\right) \longrightarrow\left(V_{\star_{S}}-K\right) \times \mathbb{C}
$$

be the induced trivialization of the normal bundle $\mathcal{N}_{X}\left(V_{\star_{S}}-K\right)=\left.\mathcal{O}_{X}\left(\sum_{i \in S} m_{i} V_{i}\right)\right|_{V_{\star_{S}}-K}$ induced by $\Phi$. Let $C \subset \cup_{i \in S-\star_{S}} T_{R, i}-K$ be a closed hypersurface transverse to $X_{\theta}$ and $V_{\star_{S}}$ and define $B \equiv C \cap V_{\star_{S}}$. Let $\Phi_{B}$ be a trivialization of the normal bundle of the contact submanifold $B \subset C$ induced by $\left.\Phi_{\star}\right|_{B}$.

Then the contact pair $\left(B \subset C, \operatorname{ker}\left(\left.\theta\right|_{C}\right), \Phi_{B}\right)$ is contactomorphic to the link of the model resolution $\left(\mathcal{O}_{X}\left(\sum_{i \in S} m_{i} V_{i}\right), \Phi, \theta\right)$. If our model resolution is graded, then both of these contact pairs have induced gradings by Lemma 5.20 and the above contactomorphism becomes a graded contactomorphism with respect to these gradings.

Proof. Choose $\check{R}>R$ smaller than the tube radius of our model resolution along $U$ so that $\check{R}<1$. Let $\alpha:[0, \check{R}] \longrightarrow[0,1]$ be a smooth function so that $\alpha^{\prime} \geq 0, \alpha(x)=x$ for all $x \leq R$ and $\alpha(x)=1$ near $\check{R}$. Define $\alpha_{\rho_{i}}: X-K \longrightarrow \mathbb{R}$ to be equal to $\alpha\left(\rho_{i}\right)$ inside $T_{\breve{R}, i}-K$ and 1 otherwise. Define

$$
f: X-K \longrightarrow \mathbb{R}, \quad f \equiv \sum_{i \in S-\star_{S}} \log \left(\alpha_{\rho_{i}}\right)
$$

Then $f$ is compatible with $\left(V_{i}\right)_{i \in S-\star_{S}}$ as in Definition 5.18, Let $c \ll-1$ and define

$$
\check{C} \equiv f^{-1}(c), \quad \check{B} \equiv \check{C} \cap V_{\star_{S}},\left.\quad \xi_{\check{C}} \equiv \operatorname{ker}(\theta)\right|_{\check{C}} .
$$

The normal bundle of $\check{B}$ inside $\check{C}$ has a natural trivialization $\Phi_{\check{B}}$ induced by the trivialization $\Phi_{\star}$. Since $d f\left(X_{\theta}\right)>0$ near $K$ and $c \ll-1$, we get that $\left(\check{B} \subset \check{C}, \xi_{\check{C}}, \Phi_{\check{B}}\right)$ is the link of our model resolution $\left(\mathcal{O}_{X}\left(\sum_{i \in S} m_{i} V_{i}\right), \Phi, \theta\right)$ by Definition 5.25 . 
Since $d f\left(X_{\theta}\right)>0$ inside $\cup_{i \in S-\star_{S}} T_{R, i}-K$ and $\cup_{i} V_{i}$ is connected, we can choose a smooth family of hypersurfaces $\left(C_{t}\right)_{t \in[0,1]}$ joining $C$ and $\check{C}$ so that $C_{t}$ is transverse to $X_{\theta}$ and $V_{\star_{S}}$ for all $t \in[0,1]$. Define $B_{t} \equiv C_{t} \cap V_{\star_{S}}$ and $\xi_{t} \equiv \operatorname{ker}\left(\left.\theta\right|_{C_{t}}\right)$. Also let $\Phi_{B_{t}}$ be the trivialization of the normal bundle of $B_{t}$ inside $C_{t}$ induced by $\Phi_{\star}$ so that $\Phi_{B_{0}}=\Phi_{B}$ and $\Phi_{B_{1}}=\Phi_{\check{B}}$. Then $\left(B_{t} \subset C_{t}, \xi_{t}, \Phi_{B_{t}}\right)$ is a smooth family of contact pairs joining $\left(B \subset C, \operatorname{ker}\left(\left.\theta\right|_{C}\right), \Phi_{B}\right)$ and $(\check{B} \subset$ $\left.\check{C}, \xi_{\check{C}}, \Phi_{\check{B}}\right)$. Therefore they are isomorphic by Lemma 5.34. Also if $\left(\mathcal{O}_{X}\left(\sum_{i \in S} m_{i} V_{i}\right), \Phi, \theta\right)$ is graded then they are graded isomorphic since all of our contact pairs have induced gradings from our model resolution by Lemma 5.20.

Lemma 5.36. The link of a (graded) model resolution $\left(\mathcal{O}_{X}\left(\sum_{i \in S} m_{i} V_{i}\right), \Phi, \theta\right)$ supports a (graded) contact open book which is contactomorphic to $O B D\left(M, \theta_{M}, \phi\right)$ where $\left(M, \theta_{M}, \phi\right)$ is the (graded) abstract contact open book associated to this model resolution as in Definition 5.33.

Proof. In this proof we will use the same notation as in Definition 5.33. We will introduce it again here for the sake of clarity. By Lemma 5.31 we can isotope our model resolution so that it admits a regularization

$$
\mathcal{R} \equiv\left(\left(\rho_{i}\right)_{i \in I},\left(\Psi_{I}\right)_{I \subset S}\right)
$$

of radius $R$ along $U$ for some relatively compact open $U$ containing $K \equiv \cup_{i \in S-\star_{S}} V_{i}$. By Lemma 5.26, the link does not change after this isotopy. Let $T_{r, I}$ be the radius $r$ tube of $V_{I}$ as in Equation (5.14). Let $\check{T}$ be a smoothing of the manifold with corners $\cup_{i \in S-\star_{S}} T_{R, i}$ as in Definition 5.33. In other words, $\check{T}$ satisfies Equation (5.38),$X_{\theta}$ points outwards along $\partial \check{T}$ and $\check{T} \subset \cup_{i \in S-\star_{S}} T_{R, i}$. Also we require that $\check{T}$ contains $\cup_{i \in S-\star_{S}} T_{3 R / 4, i}$. Define

$$
\pi_{\Phi}: \check{T} \longrightarrow \mathbb{C},\left.\quad \pi_{\Phi} \equiv \Phi_{2} \circ s_{\left(m_{i}\right)_{i \in S}}\right|_{\check{T}}
$$

where $\Phi_{2}: \mathcal{O}_{X}\left(\sum_{i \in S} m_{i} V_{i}\right) \longrightarrow \mathbb{C}$ is the composition of $\Phi$ with the natural projection map $X \times \mathbb{C} \longrightarrow \mathbb{C}$. Then we can assume that

$$
\left(M, \theta_{M}\right) \equiv\left(\pi_{\Phi}^{-1}(\epsilon),\left.\theta\right|_{\pi_{\Phi}^{-1}(\epsilon)}\right)
$$

for $\epsilon>0$ small enough so that Equation (5.37) is satisfied. Let $\omega$ be the symplectic form associated to our model resolution. Here $\phi: M \longrightarrow M$ is the monodromy map around the loop

$$
[0,1] \longrightarrow \partial \mathbb{D}(\epsilon), \quad s \longrightarrow \epsilon e^{2 \pi i s}
$$

with respect to the symplectic connection associated to $\omega$. Then $(M, \theta, \phi)$ is the abstract open book associated to our model resolution so long as $\epsilon>0$ is sufficiently small.

Define

Let

$$
L_{r} \equiv \cup_{i \in S-\star_{S}} T_{r, i}
$$

$$
\left.\Phi_{\star} \equiv \Phi\right|_{V_{\star_{S}}-K}: \mathcal{N}_{X}\left(V_{\star_{S}}-K\right) \longrightarrow\left(V_{\star_{S}}-K\right) \times \mathbb{C}
$$

be the trivialization of the normal bundle $\mathcal{N}_{X}\left(V_{\star_{S}}-K\right)=\left.\mathcal{O}_{X}\left(\sum_{i \in S} m_{i} V_{i}\right)\right|_{V_{\star} S}-K$ induced by $\Phi$ as defined in the statement of Lemma 5.35] and let $\Phi_{\star, 2}$ be the composition of $\Phi_{\star}$ with the natural projection map $V_{\star_{S}-K} \times \mathbb{C} \longrightarrow \mathbb{C}$. Let

$$
P_{\star_{S}}: \operatorname{Im}\left(\Psi_{\star_{S}}\right) \longrightarrow V_{\star_{S}}, \quad P_{\star_{S}} \equiv \pi_{\mathcal{N}_{X} V_{\star_{S}}} \circ \Psi_{\star_{S}}^{-1}
$$

be the natural projection map and $(r, \vartheta)$ polar coordinates on $\mathbb{C}$. Let $W: L_{R} \cap V_{\star_{S}} \longrightarrow[0,1]$ be a smooth function equal to 0 inside $L_{4 R / 5} \cap V_{\star_{S}}$ and equal to 1 inside $\left(L_{R}-L_{5 R / 6}\right) \cap V_{\star_{S}}$ and define

$$
\widetilde{W}: T_{R, \star_{S}} \cap L_{R} \longrightarrow \mathbb{R}, \quad \widetilde{W} \equiv W \circ P_{\star_{S}} .
$$


We now define $\theta_{t} \in \Omega^{1}\left(\left(\left(T_{R, \star_{S}} \cap L_{R}\right) \cup L_{4 R / 5}\right)-K\right)$ for $t \in[0,1]$ to be $\theta$ inside $L_{4 R / 5}-K$ and equal to

$$
(1-t) \theta+t\left((1-\widetilde{W}) \theta+\widetilde{W}\left(P_{\star_{S}}^{*}\left(\left.\theta\right|_{V_{\star_{S}}-K}\right)+\frac{1}{2} \rho_{\star_{S}}\left(\Psi_{\star_{S}}^{-1}\right)^{*} \Phi_{\star, 2}^{*}(d \vartheta)\right)\right)
$$

inside $T_{R, \star_{S}} \cap L_{R}-K$. For $R_{1}>0$ small enough with respect to $R$, we get that $d \theta_{t}$ is a symplectic form inside $L \equiv L_{4 R / 5} \cup\left(L_{R} \cap T_{R_{1}, \star_{s}}\right)$ and $d \theta_{t}$ restricted to $\pi_{\Phi}^{-1}(x) \cap L$ is a symplectic form for all $x \in \mathbb{C}-0$ and $t \in[0,1]$.

Let $\kappa: V_{\star_{S}} \longrightarrow \mathbb{R}$ be a smooth function which is negative in the interior of $\check{T} \cap V_{\star_{S}}$ and positive outside $\check{T} \cap V_{\star_{S}}$ and so that $\kappa^{-1}(0)=\partial \check{T} \cap V_{\star_{S}}$ is a regular level set. We can assume that our perturbation $\check{T}$ from Definition 5.33 is small enough so that $\partial \check{T} \subset L_{R}-L_{5 R / 6}$. Choose a constant $\check{\delta}>0$ small enough so that $\kappa^{-1}(-\check{\delta}, 0] \subset L_{R}-L_{5 R / 6}$ and so that $X_{\theta_{t}}^{d \theta_{t}}$ is transverse to $\widetilde{\kappa}^{-1}(s) \cap L$ for all $s \in(-\check{\delta}, \check{\delta})$. Define

$$
\widetilde{\kappa}: \operatorname{Im}\left(\Psi_{\star_{S}}\right) \longrightarrow \mathbb{R}, \quad \widetilde{\kappa} \equiv \kappa \circ P_{\star_{S}} .
$$

Define $\delta \equiv 1-e^{-\check{\delta}}$. Let

$$
h_{1}, h_{2}:[0, \delta) \longrightarrow \mathbb{R}
$$

be smooth functions satisfying:

(1) $h_{1}^{\prime}(r)<0, h_{2}^{\prime}(r) \geq 0$ for all $r>0$,

(2) $h_{1}(r)=1-r^{2}$ and $h_{2}(r)=\frac{1}{2} r^{2}$ for $r$ near 0 ,

(3) $h_{1}(r)=1-r$ and $h_{2}(r)=\epsilon^{2}$ for $r$ in $[\delta / 2, \delta)$.

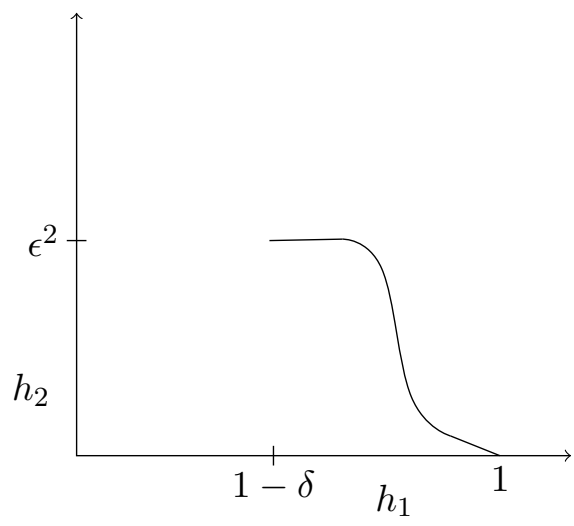

Now define

$$
C \equiv\left(\pi_{\Phi}^{-1}\left(\partial \mathbb{D}_{\epsilon}\right)-\widetilde{\kappa}^{-1}((-\check{\delta}, 0])\right) \cup \bigcup_{s \in[0, \delta]}\left(\rho_{\star_{S}}^{-1}\left(h_{2}(s)\right) \cap \widetilde{\kappa}^{-1}\left(\log \left(h_{1}(s)\right)\right)\right) .
$$

This is a smooth hypersurface in $X$ since $\Phi$ is radius $R$ compatible with $\mathcal{R}$ along $U$ and since $\epsilon>0$ can be made small enough so that $\epsilon<3 R / 4$. We can also ensure that $\epsilon>0$ is small enough so that $\pi_{\Phi}^{-1}\left(\mathbb{D}_{\epsilon}\right) \subset L$. This ensures that $d \theta_{t}$ is a symplectic form near $\pi_{\Phi}^{-1}\left(\mathbb{D}_{\epsilon}\right)$ and that $d \theta_{t}$ restricted to the fibers of $\left.\pi_{\Phi}\right|_{\pi_{\Phi}^{-1}\left(\partial \mathbb{D}_{\epsilon}\right)}$ is a symplectic form for all $t$.

Define $B \equiv C \cap V_{\star_{S}}$. This is also equal to $\check{T} \cap V_{\star_{S}}=\kappa^{-1}(0)$. For $R$ small enough, we have that $\left(C,\left.\operatorname{ker}\left(\theta_{t}\right)\right|_{C}\right)$ is a smooth family of contact submanifolds of $X$. The trivialization $\Phi_{\star}$ gives us a trivialization $\Phi_{B, t}$ of the normal bundle of the contact submanifold $B$ inside $\left(C,\left.\operatorname{ker}\left(\theta_{t}\right)\right|_{C}\right)$ since $C$ is transverse to $V_{\star_{S}}$. Hence we get a smooth family of contact pairs

$$
P_{t} \equiv\left(B \subset C,\left.\operatorname{ker}\left(\theta_{t}\right)\right|_{C}, \Phi_{B, t}\right)
$$


which are all contactomorphic by Lemma 5.34. Also by Lemma 5.35, the contact pair $P_{0}$ is contactomorphic to the link of $\left(\mathcal{O}_{X}\left(\sum_{i \in S} m_{i} V_{i}\right), \Phi, \theta\right)$ for $R$ small enough and hence $P_{1}$ is contactomorphic to the link of $\left(\mathcal{O}_{X}\left(\sum_{i \in S} m_{i} V_{i}\right), \Phi, \theta\right)$. Therefore to complete this Lemma, it is sufficient to show that the contact pair $P_{1}$ is contactomorphic to the contact pair associated to $O B D\left(M, \theta_{M}, \phi\right)$. In fact since $\left(M,\left.\theta_{t}\right|_{M}\right)$ is a smooth family of Liouville domains and since the monodromy map of $\pi_{\Phi}$ around the path (5.39) with respect to the fiberwise symplectic 2 -form $\left.d \theta_{t}\right|_{\pi_{\Phi}^{-1}(\partial \mathbb{D}(\epsilon)}$ is equal to $\phi$ for all $t$, it is sufficient for us to show that the contact pair $P_{1}$ is contactomorphic to the contact pair associated to $O B D\left(M,\left.\theta_{1}\right|_{M}, \phi\right)$. Note that there is a resemblance between the construction of $C$ and the construction of $O B D\left(M,\left.\theta_{1}\right|_{M}, \phi\right)$ from Definition 3.14. We will now make this precise.

The contact pair $P_{1}$ can be constructed as follows: Define $V \equiv \pi_{\Phi}^{-1}\left(\partial \mathbb{D}_{\epsilon}\right)-\widetilde{\kappa}^{-1}((\log (1-$ $\left.\left.\left.\frac{\delta}{2}\right), 0\right]\right)$. Let $T_{\phi}=M \times[0,1] / \sim$ be the mapping torus of $\phi$. We have a diffeomorphism $\Phi: T_{\phi} \longrightarrow \pi_{\Phi}^{-1}\left(\partial \mathbb{D}_{\epsilon}\right)$ sending $(x, s)$ to the parallel transport of $x \in M$ along $\partial \mathbb{D}(\epsilon)$ in the counter clockwise direction from $\epsilon \in \partial \mathbb{D}(\epsilon)$ to $\epsilon e^{i s} \in \partial \mathbb{D}(\epsilon)$ with respect to the 2-form $\left.d \theta_{1}\right|_{\pi_{\Phi}^{-1}(\partial \mathbb{D}(\epsilon))}$. Hence we will assume that $T_{\phi}=\pi_{\Phi}^{-1}\left(\partial \mathbb{D}_{\epsilon}\right)$ under the identification $\Phi$ and that $V$ is naturally a subset of $T_{\phi}$. Since $\phi$ has compact support inside $M$, we have the standard collar neighborhood

$$
(1-\delta, 1] \times \partial M \times(\mathbb{R} / \mathbb{Z}) \subset T_{\phi}
$$

as in Definition 3.13 (here $\delta>0$ is the same small constant defined above, which might have to be made smaller). We can choose $\kappa$ so that $\left.e^{\widetilde{\kappa}}\right|_{T_{\phi}}$ is the natural projection to $(1-\delta, 1]$ in the neighborhood (5.42). This means that $\theta_{1}$ restricted to the region (5.42) is equal to $e^{\widetilde{\kappa}} \alpha_{M}+\pi \epsilon^{2} d t$ where $t$ parameterizes $\mathbb{R} / \mathbb{Z}$ and where $\alpha_{M}=\left.\theta_{1}\right|_{\partial M}$ by Equation (5.40).

Using the diffeomorphism $\Phi$ and definition (5.41) of $C$, we have that $C$ is naturally diffeomorphic to

$$
\check{C} \equiv(\partial M \times \mathbb{D}(\delta)) \sqcup V / \sim
$$

where $\sim$ identifies $(x, z) \in \partial M \times\left(\mathbb{D}(\delta)-\mathbb{D}\left(\frac{\delta}{2}\right)\right)$ with

$$
\left(1-|z|, x, \frac{1}{2 \pi} \arg (z)\right) \in(1-\delta, 1-\delta / 2] \times \partial M \times(\mathbb{R} / \mathbb{Z}) \subset V .
$$

Because $\theta_{1}$ restricted to $T_{R, \star_{S}} \cap\left(L_{R}-L_{5 R / 6}\right)$ is equal to $P_{\star_{S}}^{*}\left(\left.\theta\right|_{V_{\star_{S}}-K}\right)+\frac{1}{2} \rho_{\star_{S}}\left(\Psi_{\star_{S}}^{-1}\right)^{*} \Phi_{\star}^{*}(d \vartheta)$ by Equation (5.40) and because $\left.P_{\star_{S}}^{*}\left(\left.\theta\right|_{V_{\star_{S}}-K}\right)\right|_{M \cap \widetilde{\kappa}^{-1}(-\check{\delta}, 0]}=e^{\widetilde{\kappa}} \alpha_{M}$ inside the cylinder (1$\delta, 1] \times \partial M \subset M$, we have that the contact form $\left.\theta_{1}\right|_{C}$ inside $\check{C}$ under the above identification is equal to

$$
\alpha_{1} \equiv\left\{\begin{array}{ll}
h_{1}(r) \alpha_{M}+\frac{1}{2} h_{2}(r) d \vartheta & \text { inside } \partial M \times \mathbb{D}(\delta / 2) \\
\theta_{1} \mid T_{\phi} & \text { inside } V
\end{array} .\right.
$$

Notice that this description of $P_{1}$ resembles the construction of the open book associated to the abstract contact open book $\left(M,\left.\theta_{1}\right|_{M}, \phi\right)$ as in Definition 3.14. All we need to do is deform the above construction until it is actually equal to $O B D\left(M,\left.\theta_{1}\right|_{M}, \phi\right)$.

We will now do this explicitly. From now on we let $t: V \longrightarrow \mathbb{R} / \mathbb{Z}$ be the coordinate $\pi_{\Phi}^{*}(\vartheta) / 2 \pi$. Since the monodromy map $\phi$ has compact support, there is a smooth function $F_{\phi}: M \longrightarrow \mathbb{R}$ so that $\phi^{*}\left(\left.\theta_{1}\right|_{M}\right)=\left.\theta_{1}\right|_{M}+d F_{\phi}$. Let $\rho:[0,1] \longrightarrow[0,1]$ be a smooth function equal to 0 near 0 and 1 near 1 . Since $T_{\phi}=M \times[0,1] / \sim$ where $\sim$ identifies $(x, 1)$ with $(\phi(x), 0)$, we have a well defined 1 -form $\left.\theta_{1}\right|_{M}+d\left(\rho(t) F_{\phi}\right)$ on $T_{\phi}$. For $s \in[0,1]$, define

$$
\alpha_{s} \in \Omega^{1}\left(T_{\phi}\right),\left.\quad \alpha_{s} \equiv(1-s) \theta_{1}\right|_{T_{\phi}}+s\left(\left.\theta_{1}\right|_{M}+d\left(\rho(t) F_{\phi}\right)\right)+c_{s} d t
$$


where $\left(c_{s}\right)_{s \in[0,1]}$ is a smooth family of constants where $c_{0}=1$ and $c_{t}$ is sufficiently large so that $\alpha_{s}$ is a contact form for all $s \in[0,1]$. Then $\left(T_{\phi}, \alpha_{1}\right)$ is the mapping torus of $\left(M,\left.\theta_{1}\right|_{M}, \phi\right)$ as in Definition 3.13 .

Choose a smooth family of functions

$$
h_{1}^{s}, h_{2}^{s}:[0, \delta) \longrightarrow[0, \infty), \quad s \in[0,1]
$$

satisfying

(1) $\left(h_{1}^{s}\right)^{\prime}(r)<0,\left(h_{2}^{s}\right)^{\prime}(r) \geq 0$ for all $r>0$,

(2) $h_{1}^{s}(r)=1-r^{2}$ and $h_{2}^{s}(r)=\frac{1}{2} r^{2}$ for $r$ near 0 ,

(3) $h_{1}^{s}(r)=1-r$ and $h_{2}^{s}(r)=(1-s) \epsilon^{2}+c_{s}$ for $r$ in $[\delta / 2, \delta)$,

(4) $h_{1}^{0}(r)=h_{1}(r)$ and $h_{2}^{0}(r)=h_{2}(r)$ for all $r \in[0, \delta)$.

Define

$$
\alpha_{1}^{s} \equiv \begin{cases}h_{1}^{s}(r) \alpha_{M}+\frac{1}{2} h_{2}^{s}(r) d \vartheta & \text { inside } \partial M \times \mathbb{D}(\delta / 2) \\ \alpha_{s} & \text { inside } V \subset T_{\phi}\end{cases}
$$

for all $s \in[0,1]$. Then $\left(C, \operatorname{ker}\left(\alpha_{1}^{s}\right)\right)_{s \in[0,1]}$ is a smooth family of contact manifolds so that $B \subset C$ is a contact submanifold. Also we have a smooth family of trivializations $\Phi_{1}^{s}$ of the normal bundle of $B$ inside $\left(C, \operatorname{ker}\left(\alpha_{1}^{s}\right)\right)$ so that $\Phi_{1}^{0}=\Phi_{B, 1}$. Therefore

$$
\check{P}_{t} \equiv\left(B \subset C, \operatorname{ker}\left(\alpha_{1}^{s}\right), \Phi_{1}^{s}\right)
$$

is a smooth family of contact pairs and so by Lemma 5.34, they are all contactomorphic. By construction, $\check{P}_{1}$ is equal to $O B D\left(M, \theta_{M}, \phi\right)$. Since $\check{P}_{1}$ is contactomorphic to $\check{P}_{0}=P_{1}$ and $P_{1}$ is contactomorphic to $P_{0}$ which in turn is contactomorphic to the link of our model resolution, we get that $O B D\left(M, \theta_{M}, \phi\right)$ is contactomorphic to the link of our model resolution.

5.5. Dynamics of abstract contact open books associated to model resolutions. In this subsection we show that the fixed points of a positive slope perturbation of the symplectomorphism associated to the graded abstract contact open book associated to a model resolution form a union of specific codimension 0 families of fixed points. We also compute the indices of these fixed points.

Definition 5.37. Let $\left(\mathcal{O}_{X}\left(\sum_{i \in S} m_{i} V_{i}\right), \Phi, \theta\right)$ be a graded model resolution with associated symplectic form $\omega$ on $X$ where $n+1=\frac{1}{2} \operatorname{dim}(X)$. Let $J$ be an $\omega$-compatible almost complex structure on $X$. By Definition [7.6, the grading on $\check{X} \equiv X-\cup_{i \in S-\star_{S}} V_{i}$ corresponds to a trivialization $\Phi:\left.\kappa_{J}\right|_{\check{X}} \longrightarrow \check{X} \times \mathbb{C}$ of the canonical bundle. Let $U$ be a small neighborhood of $\cup_{i \in S-\star_{S}} V_{i}$ which deformation retracts on to $V_{i}$. Choose a smooth section $s$ of $\kappa_{J}$ which is transverse to 0 and so that $\left.\Phi \circ s\right|_{\check{X}-U}$ is a non-zero constant section of $(\check{X}-U) \times \mathbb{C}$. By a MayorVietoris argument, the homology group $H_{2 n}\left(\cup_{i \in S-\star_{S}} V_{i} ; \mathbb{Z}\right)=H_{2 n}(U ; \mathbb{Z})$ is freely generated by the fundamental classes $\left[V_{i}\right]$ of $V_{i}$. Let $\left[s^{-1}(0)\right] \in H_{2 n}(U)$ be the homology class represented by the zero set. Then $\left[s^{-1}(0)\right]=\sum_{i \in S-\star_{S}} a_{i}\left[V_{i}\right]$ for unique numbers $a_{i} \in \mathbb{Z}, i \in S-\star_{S}$. The discrepancy of $V_{i}$ is defined to be $a_{i}$ for all $i \in S-\star_{S}$.

In the case of Example 5.14, the discrepancy and multiplicity of $E_{i}$ as defined in Definition 5.37 is identical to the discrepancy and multiplicity of $f$ along $E_{i}$ as in Definition 2.1. Similarly we have a notion of multiplicity $m$ separating resolution as in Definition 2.2 for model resolutions which coincide in the case of Example 5.14;

Definition 5.38. A model resolution $\left(\mathcal{O}_{X}\left(\sum_{i \in S} m_{i} V_{i}\right), \Phi, \theta\right)$ is called a multiplicity $m$ separating resolution if $m_{i}+m_{j}>m$ for all $i, j \in S$ satisfying $V_{i} \cap V_{j} \neq \emptyset$. 
Definition 5.39. Let $\left(\mathcal{O}_{X}\left(\sum_{i \in S} m_{i} V_{i}\right), \Phi, \theta\right)$ be a model resolution. Let $i \in S-\star_{S}$. Define $V_{i}^{o} \equiv V_{i}-\cup_{j \in S-i} V_{j}$ and $X_{i} \equiv X-\cup_{j \in S-i} V_{j}$. Let $U_{i}$ be an open neighborhood of $V_{i}^{o}$ inside $X_{i}$ which deformation retracts on to $V_{i}^{o}$ and let $\iota_{i}: U_{i}-V_{i}^{o} \longrightarrow U_{i}$ be the natural inclusion map. Let $s_{\left(m_{i}\right)_{i \in S}}$ be the canonical section of $\mathcal{O}_{X}\left(\sum_{i \in S} m_{i} V_{i}\right)$ as in Equation (5.12). Let $\Phi_{2}: \mathcal{O}_{X}\left(\sum_{i \in S} m_{i} V_{i}\right) \longrightarrow \mathbb{C}$ is the composition of $\Phi$ with the natural projection map to $\mathbb{C}$. Define

$$
Q_{i}: U_{i}-V_{i}^{o} \longrightarrow \mathbb{C}^{*}, \quad Q_{i}(x):=\Phi_{2} \circ s_{\left(m_{i}\right)_{i \in S}}(x) .
$$

Then the natural $m_{i}$-fold covering of $V_{i}^{o}$ is the $m_{i}$-fold covering of $V_{i}^{o}$ given by a disjoint union of covers diffeomorphic to the cover corresponding to the normal subgroup

$$
G_{i}:=\left(\iota_{i}\right)_{*}\left(\operatorname{ker}\left(\left(Q_{i}\right)_{*}\right)\right) \subset \pi_{1}\left(U_{i}\right)=\pi_{1}\left(V_{i}^{o}\right)
$$

and the number of such covers is $m_{i}$ divided by the index of $G_{i}$ in $\pi_{1}\left(V_{i}^{o}\right)$ (see Lemma 5.40 below). Such a cover does not depend on the choice of neighborhood $U_{i}$. In fact it is an invariant of the model resolution up to isotopy.

Lemma 5.40. The index of $G_{i}$ divides $m_{i}$.

The proof of this lemma also gives us a geometric interpretation of $\widetilde{V}_{i}^{o}$.

Proof. After an isotopy, we can assume that our model resolution admits a regularization

$$
\mathcal{R} \equiv\left(\left(\rho_{j}\right)_{j \in I},\left(\Psi_{I}\right)_{I \subset S}\right)
$$

of radius $R$ along $U$ for some relatively compact open $U$ containing $\cup_{j \in S-\star_{S}} V_{i}$. Let $T_{r, i}$ be the radius $r$ tube of $V_{i}$ as in Equation (5.14) for some $r<3 R / 4$. We can assume that the open neighborhood $U_{i}$ from Definition 5.39 is equal to $T_{r, i}-\cup_{j \in S-i} V_{j}$. We have that our map $Q_{i}$ is equal to

$$
Q_{i}: U_{i}-V_{i}^{o} \longrightarrow \mathbb{C}^{*}, \quad Q_{i}(x) \equiv \Phi_{2} \circ s_{\left(m_{i}\right)_{i \in S}}(x) .
$$

Define $\mathbb{D}(\epsilon)^{*} \equiv \mathbb{D}(\epsilon)-0$ where $\mathbb{D}(\epsilon) \subset \mathbb{C}$ is the $\epsilon$-disk. Then $Q_{i}$ restricted to $Q_{i}^{-1}\left(\mathbb{D}(\epsilon)^{*}\right)$ for $\epsilon>0$ small enough is a fibration whose fibers are smooth manifolds with corners. Combining this with the fact that $\pi_{2}\left(\mathbb{D}(\epsilon)^{*}\right)=0$ we get that the map

$$
\pi_{1}\left(Q_{i}^{-1}(\epsilon)\right) \longrightarrow \operatorname{ker}\left(\left(\left.Q_{i}\right|_{Q_{i}^{-1}\left(\mathbb{D}(\epsilon)^{*}\right)}\right)_{*}\right)=\operatorname{ker}\left(\left(Q_{i}\right)_{*}\right)
$$

is an isomorphism by a fibration long exact sequence argument. Therefore the natural map

$$
\pi_{1}\left(Q_{i}^{-1}(\epsilon)\right) \longrightarrow \pi_{1}\left(U_{i}\right)
$$

has image $G_{i}$. Also for $0<\epsilon \ll r \ll 1$, the map

$$
P: Q_{i}^{-1}(\epsilon) \longrightarrow V_{i}^{o},\left.\quad P(x) \equiv \pi_{\mathcal{N}_{X}} \circ \Psi_{i}^{-1}\right|_{Q_{i}^{-1}(\epsilon)}(x)
$$

is covering map of order $m_{i}$ over $\operatorname{Im}(P)$ and $V_{i}^{o}$ is homotopic to the image $\operatorname{Im}(P)$. Hence the index of $G_{i}$ divides $m_{i}$.

Theorem 5.41. Let $m \in \mathbb{N}_{>0}$ and let $\left(\mathcal{O}_{X}\left(\sum_{i \in S} m_{i} V_{i}\right), \Phi, \theta\right)$ be a graded model resolution that is also a multiplicity $m$ separating resolution and define $V_{i}^{o} \equiv V_{i}-\cup_{j \in S-i} V_{j}$ for all $i \in S$ and define

$$
S_{m} \equiv\left\{i \in S-\star_{S}: m_{i} \text { divides } m\right\} \text {. }
$$

Let $a_{i}$ be the discrepancy of $V_{i}$ for each $i \in S-\star_{S}$. Then there is a graded abstract contact open book $\left(M, \theta_{M}, \phi\right)$ so that the contact pair associated to it is graded contactomorphic to the link of our model resolution. Also there is small positive slope perturbation $\check{\phi}$ of $\phi^{m}$ so that the fixed point set of $\check{\phi}$ is a disjoint union of codimension 0 families of fixed points $\left(B_{i}\right)_{i \in S_{m}}$ satisfying 
(1) $H^{*}\left(B_{i} ; \mathbb{Z}\right)=H^{*}\left(\widetilde{V}_{i}^{o} ; \mathbb{Z}\right)$ where $\widetilde{V}_{i}^{o}$ is the natural $m_{i}$-fold covering of $V_{i}^{o}$ as in Definition 5.39 ,

(2) the action of $B_{i}$ is equal to $-m_{i} w_{i}-\pi\left(m_{i}-m\right) \epsilon^{2}$ where $w_{i}$ wrapping number of $\theta$ around $V_{i}$ and

(3) $C Z\left(\check{\phi}, B_{i}\right)=2 k_{i}\left(a_{i}+1\right)$ where $k_{i} \equiv \frac{m}{m_{i}}$

for all $i \in S-\star_{S}$.

Proof of Theorem 5.41. In this proof we will use the same notation as in Definition 5.33. We will introduce it again here for the sake of clarity. After an isotopy, we can assume that our model resolution admits a regularization

$$
\mathcal{R} \equiv\left(\left(\rho_{i}\right)_{i \in I},\left(\Psi_{I}\right)_{I \subset S}\right)
$$

of radius $R$ along $U$ for some relatively compact open $U$ containing $K \equiv \cup_{i \in S-\star_{S}} V_{i}$ since by Lemma [5.26, the link does not change after this isotopy. Our abstract contact open book $\left(M, \theta_{M}, \phi\right)$ will be the graded abstract contact open book associated to this model resolution as in Definition 5.33. By Lemma 5.36, the link of $O B D\left(M, \theta_{M}, \phi\right)$ is contactomorphic to the link of our model resolution.

We now wish to show that $\phi$ satisfies properties (11)-(3) listed in the statement of this theorem. To do this, we need to recall the construction of $\left(M, \theta_{M}, \phi\right)$. Let $T_{r, I}$ be the radius $r$ tube of $V_{I}$ as in Equation (5.14) and let $T_{r, I}^{o}$ be the interior of $T_{r, I}$. Let $\check{T}$ be a smoothing of the manifold with corners $\cup_{i \in S-\star_{S}} T_{R, i}$ as in Definition 5.33. In other words, $\check{T}$ satisfies Equation (5.38),$X_{\theta}$ points outwards along $\partial \check{T}, \check{T} \subset \cup_{i \in S-\star_{S}} T_{R, i}$ and $\cup_{i \in S-\star_{S}} T_{3 R / 4, i}$ is contained in the interior of $\check{T}$. Define

$$
\pi_{\Phi}: \check{T} \longrightarrow \mathbb{C},\left.\quad \pi_{\Phi} \equiv \Phi_{2} \circ s_{\left(m_{i}\right)_{i \in S}}\right|_{\breve{T}}
$$

where $\Phi_{2}: \mathcal{O}_{X}\left(\sum_{i \in S} m_{i} V_{i}\right) \longrightarrow \mathbb{C}$ is the composition of $\Phi$ with the natural projection map $X \times \mathbb{C} \longrightarrow \mathbb{C}$. Then we can assume that

$$
\left(M, \theta_{M}\right) \equiv\left(\pi_{\Phi}^{-1}(\epsilon),\left.\theta\right|_{\pi_{\Phi}^{-1}(\epsilon)}\right)
$$

for some small $\epsilon>0$. We will assume that $\epsilon>0$ is small enough so that $M \subset \cup_{i \in S} T_{R / 4, i}$ and the fibers of $\left.\pi_{\Phi}\right|_{\pi_{\Phi}^{-1}(\mathbb{D}(\epsilon))}$ are symplectic by Lemma [5.32. Let $\omega$ be the associated symplectic form of our model resolution. Define $\phi: M \longrightarrow M$ to be the monodromy map around the loop

$$
[0,1] \longrightarrow \partial D(\epsilon), \quad t \longrightarrow e^{2 \pi i t}
$$

with respect to the symplectic connection associated to $\omega$.

First of all, we will compute the fixed points of the map $\phi^{m}$. To do this, we will show that they correspond to certain periodic orbits of the flow of a Hamiltonian on $\check{T}$. Define

$$
H: \operatorname{Dom}\left(\pi_{\Phi}\right)=\check{T} \rightarrow \mathbb{R}, \quad H(x)=|F(x)|, \quad \forall x \in \check{T} .
$$

It is sufficient for us to find the periodic orbits of $X_{H}$ starting inside $M$ which map under $\pi_{\Phi}$ to loops in $\mathbb{C}^{*}$ which wrap around 0 exactly $m$ times in the anti-clockwise direction. This is because there is a $1-1$ correspondence between fixed points of $\phi^{m}$ and such orbits. This correspondence sends a fixed point $p$ of $\phi^{m}$ to the unique flowline of $X_{H}$ starting and ending at $p$ whose image under $\pi_{\Phi}$ wraps around 0 exactly $m$ times in the anti-clockwise direction.

Define $\check{T}_{R, I} \equiv T_{\frac{3 R}{4}, I}^{o}-\cup_{i \in S-I} T_{\frac{3 R}{4}, i}^{o}$ for each $I \subset S$. Since $M \subset \cup_{I \subset S} \check{T}_{R, I}$, it is sufficient for us to calculate the fixed points of $\phi^{m}$ inside $M \cap \check{T}_{R, I}$ for each $I \subset S$. Therefore we will now compute the periodic orbits of $X_{H}$ starting inside $M \cap \check{T}_{R, I}$ for all $I \subset S$ which project to loops 
in $\mathbb{C}^{*}$ wrapping $m$ times around 0 in an anti-clockwise direction. Let $a_{R}:[0, \infty) \rightarrow[0, \infty)$ be the smooth function defined in Definition 5.9. In other words, $a_{R}$ satisfies:

(1) $a_{R}^{\prime}(x)>0$ for $x \in[0,3 R / 4)$,

(2) $a_{R}(x)=x$ for $x \leq R / 4$

(3) $a_{R}(x)=1$ for $x \geq 3 R / 4$.

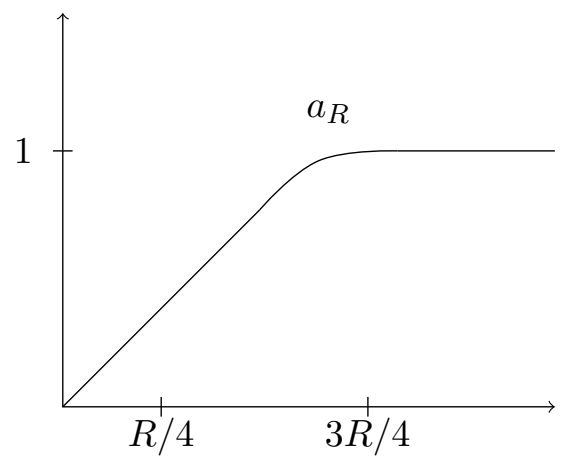

Define

$$
b_{R}:[0, \infty) \rightarrow[0, \infty), \quad b_{R}(x) \equiv \sqrt{a_{R}(x)}
$$

Let

$$
p_{I}: T_{R, I} \rightarrow V_{I}, \quad p_{I}(x) \equiv \pi_{\mathcal{N}_{X} V_{I}}\left(\Psi_{I}^{-1}(x)\right)
$$

be the natural projection map. Inside $\check{T}_{R, I}$ we have that

$$
H(x)=\prod_{i \in I}\left(b_{R}\left(\rho_{i}(x)\right)\right)^{m_{i}}, \quad \forall x \in \check{T}_{R, I}
$$

since the bundle trivialization $\Phi$ is radius $R$ compatible with our regularization $\mathcal{R}$ along $T_{R, I}$. Hence

$$
\left.X_{H}\right|_{x}=\left.\sum_{i \in I}\left(m_{i} b_{R}^{\prime}\left(\rho_{i}(x)\right) b_{R}\left(\rho_{i}(x)\right)^{m_{i}} \prod_{j \in I-i} b_{R}\left(\rho_{j}(x)\right)^{m_{j}}\right) X_{\rho_{i}}\right|_{x} \quad \forall x \in \check{T}_{R, I} .
$$

This means that all the periodic orbits of $X_{H}$ starting inside $\check{T}_{R, I}$ are contained inside the fibers of $p_{I}$ since the vector fields $X_{\rho_{i}}$ are tangent to these fibers. Also since $b_{R}\left(\rho_{i}(x)\right)>0$ and $b_{R}^{\prime}\left(\rho_{i}(x)\right)>0$ for all $x \in \check{T}_{R, I}$ and all $i \in I$, we have that any disk contained inside a fiber of $p_{I}$ bounding any such orbit must intersect $V_{i}$ positively for all $i \in I$. This implies that the projection of this orbit to $\mathbb{C}^{*}$ wraps around 0 more than $m$ times if $|I|>1$ since our model resolution is a multiplicity $m$ separating resolution. This means that if the set of periodic orbits of $X_{H}$ starting inside $M \cap \check{T}_{R, I}$ whose image in $\mathbb{C}^{*}$ wraps $m$ times around 0 is non-empty then $|I|=1$. Hence all fixed points of $\phi^{m}$ are contained inside $\cup_{i \in S} M \cap \check{T}_{R, i}$. Similar reasoning ensures that $i \in S_{m} \cup\left\{\star_{S}\right\}$ and that the set of fixed points of $\phi^{m}$ inside $T_{R, i}$ is $B_{i} \equiv M \cap \check{T}_{R, i}$ for all such $i$.

By Lemma 5.42 below with $W=T_{R, i}, h=\left.\pi \rho_{i}\right|_{T_{R, i}}, B_{1}=h^{-1}(\pi \sqrt{\epsilon}), B_{2}=T_{R, i} \cap\left\{\left|\pi_{\Phi}\right|=\epsilon\right\}$, and $\left.f_{j} \equiv \frac{1}{2 \pi} \arg \left(\pi_{\Phi}\right)\right|_{B_{j}}$ for $j=1,2$, we have that $B_{i}$ is a codimension 0 family of fixed points of $\phi^{m}$ for all $i \in S_{m}$. Since $B_{i}$ is homotopic to $\pi_{\Phi}^{-1}(\epsilon) \cap T_{R, i}$ which in turn is homotopic to the fiber $Q_{i}^{-1}(\epsilon)$ constructed in the proof of Lemma 5.40 we have $H^{*}\left(B_{i} ; \mathbb{Z}\right)=H^{*}\left(\widetilde{V}_{i}^{o} ; \mathbb{Z}\right)$ for all $i \in S-\star_{S}$.

We now need to construct a small positive slope perturbation $\check{\phi}$ of $\phi^{m}$ without creating any extra fixed points so that $B_{\star_{S}}$ disappears and so that $\phi=\check{\phi}$ near $\cup_{i \in S_{m}} B_{i}$. Since $B_{\star_{S}}$ 
is a codimension 0 family of fixed points of $\phi$, there is a neighborhood $N_{\star_{S}}$ of $B_{\star_{S}}$ and a Hamiltonian $H_{\star_{S}}: N_{\star_{S}} \longrightarrow(-\infty, 0]$ so that $\phi^{m}$ is the time 1-flow of $H_{\star_{S}}$ inside $N_{\star_{S}}$ and so that $B_{\star_{S}}=H_{\star_{S}}^{-1}(0)$. Choose $\delta_{\star}>0$ small enough so that $H_{\star_{S}}$ has no $q$-periodic orbits inside $H_{\star_{S}}^{-1}\left(-\delta_{\star}, 0\right)$ for all $q \in[0,2]$. Since the vector field (5.45) is tangent to the fibers of $p_{I}$ inside $T_{R, I} \cap T_{3 R / 4, \star_{S}}$ for all $I \subset S$ and since $\Psi_{I}$ is a regularization, we have that $H_{\star_{S}}$ must be a function of the variables $\left(\rho_{i}\right)_{i \in I}$ inside $T_{R, I} \cap T_{3 R / 4, \star_{S}}$ only. This implies that we can construct a smooth function $\check{b}: N_{\star S} \longrightarrow \mathbb{R}$ for $\delta_{\star}>0$ small enough so that

- $\breve{b}=F \circ H_{\star_{S}}$ for some smooth function $F: \mathbb{R} \longrightarrow \mathbb{R}$ inside $H_{\star_{S}}^{-1}\left(\left[-\delta_{\star_{S}},-\delta_{\star_{S}} / 3\right]\right)$ where $F \circ H_{\star_{S}}=H_{\star_{S}}$ near $H_{\star_{S}}^{-1}\left(-\delta_{\star_{S}}\right)$,

- $\check{b}$ is $C^{2}$ small inside $H_{\star_{S}}^{-1}\left(\left[-\delta_{\star_{S}} / 3,0\right]\right)$

- $\check{b}=\delta r_{M}$ near $\partial M$ where $r_{M}$ is the radial coordinate on $M$ and

- $\breve{b}$ has no critical points.

This implies that the time 1-flow of $\check{b}$ has no fixed points inside $N_{\star_{S}}$ and is equal to $H_{\star_{S}}$ outside a compact subset of $N_{\star_{S}}$. Define $\check{\phi}$ to be equal to $\phi^{m}$ outside $N_{\star_{S}}$ and the time 1-flow of $\breve{b}$ inside $N_{\star_{S}}$. This is a positive slope perturbation of $\phi^{m}$ so that the set of fixed points of $\check{\phi}$ is $\cup_{i \in S-\star_{S}} B_{i}$ and $\check{\phi}=\phi^{m}$ in a neighborhood of these fixed points.

Next we need to compute the action of $B_{i}$ for each $i \in S-\star_{S}$. Let $p \in B_{i}$ and let $\gamma: \mathbb{R} / \mathbb{Z} \rightarrow \check{T}$ be the unique loop starting at $p \in M$ which is symplectically orthogonal to the fibers of $\pi_{\Phi}$ and satisfying $\pi_{\Phi} \circ \gamma(t)=e^{2 i \pi m t}$ for all $t \in \mathbb{R}$. Then the action of $p$ is equal to $-\int_{0}^{1} \gamma^{*} \theta+\pi m \epsilon^{2}=-m_{i} w_{i}-\pi\left(m_{i}-m\right) \epsilon^{2}$.

We now need to compute the Conley-Zehnder index of $B_{i}$ for each $i \in S_{m}$. Fix $i \in S_{m}$ and let $p \in B_{i} \subset M$. Let $\gamma: \mathbb{R} / \mathbb{Z} \rightarrow \check{T}$ be the unique loop starting at $p \in M$ which is symplectically orthogonal to the fibers of $\pi_{\Phi}$ and satisfying $\pi_{\Phi} \circ \gamma(t)=e^{2 i \pi m t}$ for all $t \in \mathbb{R}$. Let $J$ be an $\omega$-compatible almost complex structure on $X$ so that $\pi_{\Phi}$ becomes $J$-holomorphic. Let

$$
\left.T^{\mathrm{ver}} \check{T} \equiv \operatorname{ker}\left(D \pi_{\Phi}\right)\right|_{\check{T}-K} \subset T(\check{T}-K)
$$

be the vertical tangent bundle. Let $\left(T^{\mathrm{ver}} \check{T}\right)^{\perp} \subset T(\check{T}-K)$ be the set of vectors which are $\omega$-orthogonal to the vertical tangent bundle. This is a $J$-holomorphic subbundle of $T(\check{T}-K)$. Let $\tau_{\mathbb{C}^{*}}: T \mathbb{C}^{*} \longrightarrow \mathbb{C}^{*} \times \mathbb{C}$ be the holomorphic trivialization which sends $\frac{\partial}{\partial \vartheta}$ to the constant section 1 and let $\tau_{\mathbb{C}^{*}, 2}: T \mathbb{C}^{*} \longrightarrow \mathbb{C}$ be the composition of $\tau_{\mathbb{C}^{*}}$ with the natural projection map $\mathbb{C}^{*} \times \mathbb{C} \longrightarrow \mathbb{C}$. We then have a trivialization

$$
\begin{gathered}
\tau^{\perp}:\left(T^{\mathrm{ver}} \check{T}\right)^{\perp} \longrightarrow(\check{T}-K) \times \mathbb{C} . \\
\tau_{T^{\perp}}(Y) \equiv\left(x, \tau_{\mathbb{C}^{*}, 2}\left(D \pi_{\Phi}(Y)\right)\right),\left.\forall Y \in\left(T^{\mathrm{ver}} \check{T}\right)^{\perp}\right|_{x}, x \in \check{T}-K .
\end{gathered}
$$

Let

$$
\left(\tau^{\perp}\right)^{*}:\left(\left(T^{\text {ver }} \check{T}\right)^{\perp}\right)^{*} \longrightarrow(\check{T} \cap \check{X}) \times \mathbb{C}
$$

be the corresponding trivialization of the dual bundle.

Let $\kappa_{J, \phi}$ be the canonical bundle of $T^{\mathrm{ver}} \check{T}$. Then we have a canonical isomorphism

$$
\left.\kappa_{J}\right|_{\breve{T}-K} \cong \kappa_{J, \phi} \otimes\left(\left(T^{\mathrm{ver}} \check{T}\right)^{\perp}\right)^{*}
$$

Since $(X-K, \omega)$ is a graded symplectic manifold, we get a natural choice of trivialization $\tau:\left.\kappa_{J}\right|_{X-K} \longrightarrow(X-K) \times \mathbb{C}$ by Definition 7.7. The trivializations $\tau$ and $\left(\tau^{\perp}\right)^{*}$ give us a trivialization $\tau^{\mathrm{ver}}: \kappa_{J, \phi} \longrightarrow(\check{T}-K) \times \mathbb{C}$ of $\kappa_{J, \phi}$ by the identity (5.46).

Let $s_{\phi}$ be a section of $\kappa_{J, \phi}$ so that it is equal to the constant section 1 with respect to our trivialization $\tau^{\text {ver }}$. Let $s$ be a section of $\kappa_{J}$ so that $s^{-1}(0)$ is transverse to 0 and contained inside a small neighborhood $N$ of $\cup_{i \in S-\star_{S}} V_{i}$ which deformation retracts on to 
$\cup_{i \in S-\star_{S}} V_{i}$ and so that $\left.\tau \circ s\right|_{X-N}$ is the constant section 1 . Then by definition $\left[s^{-1}(0)\right]$ is a homology class homologous to $\sum_{i \in S} a_{i}\left[V_{i}\right]$. Now define $\check{T}_{\epsilon} \equiv \pi_{\Phi}^{-1}(\partial \mathbb{D}(\epsilon))$. By construction, $\left.\tau^{\mathrm{ver}}\right|_{\breve{T}_{\epsilon}}$ is homotopic to the induced trivialization from Lemma 5.20 (after identifying the contact hyperplane distribution in $\check{T}_{\epsilon}$ with $T^{\mathrm{ver}} \check{T}$ using an isotopy between these symplectic subbundles).

We can choose $J$ so that near the image $\gamma$, the Hamiltonian flow $\phi_{t}^{\frac{1}{2} \rho_{i}}: T_{R, i} \longrightarrow T_{R, i}$ of $\left.\frac{1}{2} \rho_{i}\right|_{T_{R, i}}$ is $J$-holomorphic. Hence on some neighborhood $N_{\gamma}$ of $\gamma$ invariant under the flow of $X_{\frac{1}{2} \rho_{i}}$, we have that $\phi_{t}^{\frac{1}{2} \rho_{i}}$ lifts to a map

$$
\widetilde{\phi}_{t}:\left.\left.\kappa_{J}\right|_{x} \rightarrow \kappa_{J}\right|_{\phi_{t}^{\frac{1}{2} \rho_{i}}(x)}
$$

for all $x \in \check{T}_{\epsilon} \cap N_{\gamma}$ given by the highest wedge power of the $J$-holomorphic bundle map $\left(\left(D \phi_{t}^{\frac{1}{2} \rho_{i}}\right)^{-1}\right)^{*}$. Also since $D \phi_{t}^{\frac{1}{2} \rho_{i}}(v) \in T^{\mathrm{ver}} \check{T}_{\epsilon}$ for all $\left.v \in T^{\mathrm{ver}} \check{T}_{\epsilon}\right|_{N_{\gamma} \cap \check{T}_{\epsilon}}$, we get an induced map

$$
\widetilde{\phi}_{t}^{\text {ver }}:\left.\left.\kappa_{J, \phi}\right|_{\check{T}_{\epsilon} \cap N_{\gamma}} \longrightarrow \kappa_{J, \phi}\right|_{\check{T}_{\epsilon} \cap N_{\gamma}} \text {. }
$$

Let $\tau_{2}:\left.\kappa_{J}\right|_{X-K} \longrightarrow \mathbb{C}$ and $\tau_{2}^{\text {ver }}: \kappa_{J, \phi} \longrightarrow \mathbb{C}$ be the composition of $\tau$ and $\tau^{\text {ver }}$ respectively with the natural projection map to $\mathbb{C}$. The winding number of the map

$$
w_{\phi}: \mathbb{R} / 2 \pi m \mathbb{Z} \longrightarrow \mathbb{C}^{*} \cong \operatorname{Aut}(\mathbb{C}, \mathbb{C}), \quad w_{\phi}(t)=\tau_{2}^{\text {ver }} \circ\left(\left.\widetilde{\phi}_{t}^{\text {ver }}\right|_{p}\right) \circ\left(\left.\tau_{2}^{\text {ver }}\right|_{\left.\kappa_{J, \phi}\right|_{p}}\right)^{-1}
$$

is equal to the winding number of the map

$$
w_{\tau}: \mathbb{R} / 2 \pi m \mathbb{Z} \longrightarrow \mathbb{C}^{*} \cong \operatorname{Aut}(\mathbb{C}, \mathbb{C}), \quad w_{\tau}(t)=\tau_{2} \circ\left(\left.\widetilde{\phi}_{t}\right|_{p}\right) \circ\left(\left.\tau_{2}\right|_{\left.\kappa_{J}\right|_{p}}\right)^{-1} .
$$

Since $\left[s^{-1}(0)\right]$ is homologous to $\sum_{i \in S-\star_{S}} a_{i}\left[V_{i}\right]$ and $\gamma(t)=\phi_{t}^{\frac{1}{2} \rho_{i}}(p)$ for all $t$, we have that the winding number of $w_{\tau}$ is equal to

$$
k_{i}\left(-1-a_{i}\right) .
$$

Hence by Lemma 7.8 and the fact that the winding number of $w_{\phi}$ is the winding number of $w_{\tau}$, we have that the Conley-Zehnder index of the fixed point $p$ of $\phi^{m}$ is minus 2 times the winding number of $w_{\tau}$ and so $C Z\left(\phi^{m}, B_{i}\right)=2 k_{i}\left(a_{i}+1\right)$.

Here is a technical lemma that was used in the proof of Theorem 5.41 above.

Lemma 5.42. Let $(W, \omega)$ be a symplectic manifold admitting a free Hamiltonian $S^{1}$-action generated by a Hamiltonian $h: W \longrightarrow \mathbb{R}$ (I.e. $\phi_{1}^{h}=i d_{W}$ and $\phi_{t}^{h}(x) \neq x$ for all $x \in W$ and $t \in(0,1))$. Let $B_{1}, B_{2} \subset W$ be two real hypersurfaces inside $W$ with maps

$$
f_{1}: B_{1} \longrightarrow \mathbb{R} / \mathbb{Z}, \quad f_{2}: B_{2} \longrightarrow \mathbb{R} / \mathbb{Z}
$$

so that

(1) the fibers of $f_{i}$ are symplectic submanifolds of $W$ and the corresponding monodromy map $\phi_{i}: f_{i}^{-1}(0) \longrightarrow f_{i}^{-1}(0)$ of $f_{i}$ around $\mathbb{R} / \mathbb{Z}$ is well defined (I.e. no points parallel transport off to infinity in finite time) for $i=1,2$,

(2) $\phi_{1}=i d_{B_{1}}, B_{1}=h^{-1}(C)$ and $B_{2} \subset h^{-1}([C, \infty))$ for some $C>0$,

(3) $B_{i}$ is invariant under our Hamiltonian $S^{1}$-action for $i=1,2$ and for all $t \in S^{1}=\mathbb{R} / \mathbb{Z}$ and $x \in B_{1}$ we have $f_{1}(t \cdot x)=f_{1}(x)+t$ and

(4) $f_{1}^{-1}(0) \cap f_{2}^{-1}(0)$ is equal to a compact codimension 0 submanifold of $f_{2}^{-1}(0)$ with boundary and corners and $\left.f_{1}\right|_{B_{1} \cap B_{2}}=\left.f_{2}\right|_{B_{1} \cap B_{2}}$.

Then $f_{1}^{-1}(0) \cap f_{2}^{-1}(0)$ is a codimension 0 family of fixed points of $\phi_{2}^{m}$ for all $m>0$. 
Proof of Lemma 5.42. Let $Q \subset B_{1}$ be an $S^{1}$ invariant relatively compact open set containing $B_{1} \cap B_{2}$, let $V \equiv Q \cap f_{1}^{-1}(0)$ and $\left.\omega_{V} \equiv \omega\right|_{V}$. For all $r_{1}, r_{2}>0$ let $A_{r_{1}, r_{2}} \subset \mathbb{C}$ be the open annulus whose inner radius is $r_{1}$ and whose outer radius is $r_{2}$ with the standard symplectic form. Let $r: \mathbb{C} \longrightarrow[0, \infty)$ be the radial function $z \longrightarrow|z|$ and $\theta: \mathbb{C}-0 \longrightarrow \mathbb{R} / 2 \pi \mathbb{Z}$ the angle coordinate. Define $\check{C} \equiv \sqrt{C / \pi}$. Let $S_{\check{C}} \subset \mathbb{C}$ be the circle of radius $\check{C}$. After shrinking $Q$ slightly we can, by an equivariant Moser theorem (see GS90), find an $S^{1}$-equivariant open set $U \subset W$ symplectomorphic to $\left(V \times A_{\check{C}-\delta, \check{C}+\delta}, \omega_{V}+\frac{1}{2} d\left(r^{2}\right) \wedge d \vartheta\right)$ so that $Q=V \times S_{\check{C}}$ and $\left.h\right|_{U}=\pi r^{2}$.

If we smoothly deform $f_{2}$ inside $B_{2}$ through fibrations whose fibers are always transverse to the line field given by $\operatorname{ker}\left(\left.\omega\right|_{B_{2}}\right)$ then the symplectic form on the fibers and the monodromy map does not change. This is because such a deformation can be realized by a flow along a vector field tangent to the line field $\operatorname{ker}\left(\left.\omega\right|_{B_{2}}\right)$. In particular, we can assume that in some small $S^{1}$ invariant neighborhood $\check{U} \subset U$ of $B_{1} \cap B_{2}$ that

$$
\left.f_{2}\right|_{\check{U} \cap B_{2}}=\left.(\theta / 2 \pi)\right|_{\check{U} \cap B_{2}} \text {. }
$$

Let $p r_{1}: V \times A_{\check{C}-\delta, \check{C}+\delta} \longrightarrow V$ be the natural projection map. Define $V_{2} \equiv f_{2}^{-1}(0) \cap \check{U}$ and $\left.\omega_{V_{2}} \equiv \omega\right|_{V_{2}}$. We can assume that $\check{U}$ is small enough so that $\left.p r_{1}\right|_{V_{2}}: V_{2} \longrightarrow V$ is a diffeomorphism onto its image. This map is also a symplectic embedding by Equation (5.47). Define

$$
H: V_{2} \longrightarrow \mathbb{R}, \quad H(x) \equiv \pi r(x)^{2}-2 \pi \check{C}=\left.h\right|_{V_{2}}-2 \pi \check{C} .
$$

Since $\omega_{V}+\frac{1}{2} d\left(r^{2}\right) \wedge d \vartheta=\omega_{V}+d h \wedge d\left(\frac{1}{2 \pi} \vartheta\right)$ inside $\check{U}$ and $\left.p r_{1}\right|_{V_{2}}$ is a symplectic embedding, we get that the vector field

$$
-X_{H}^{\omega_{V_{2}}}+2 \pi \frac{\partial}{\partial \vartheta}
$$

is tangent to $\check{U} \cap B_{2}$ and lies in the kernel of $\left.\omega\right|_{B_{2} \cap \check{U}}$. Then for all $m>0, \phi_{2}^{m}$ is equal to the time 1 flow of $-m H$ near $B_{1} \cap f_{2}^{-1}(0)$ inside the symplectic manifold $\left(V_{2}, \omega_{V_{2}}\right)$. Hence $B_{1} \cap f_{2}^{-1}(0)$ is a codimension 0 family of fixed points of $\phi_{2}$.

\section{Proof of Theorem 1.2 and Corollary 1.3.}

Proof of Theorem 1.2. Let $L \equiv\left(L_{f} \subset S_{\epsilon}, \xi_{S_{\epsilon}}, \Phi_{f}\right)$ be the contact pair associated to $f$ with the standard grading as in Example 3.8. Let $\left(\mathcal{O}_{X}\left(\sum_{i \in S} m_{i} E_{i}\right), \Phi, \theta\right)$ be a graded model resolution coming from the log resolution $\pi: Y \longrightarrow \mathbb{C}^{n+1}$ as in Example 5.14, The wrapping number of $\theta$ around $E_{j}$ is $w_{j}$ for all $j \in S-\star_{S}$. By using the function $|z|^{2}$ on $\mathbb{C}^{n+1}$ combined with Lemma 5.34, one can show that the link of this model resolution is contactomorphic to $L$. Hence Theorem 1.2 follows from Theorem 5.41, (HF2) and (HF3),

Lemma 6.1. Suppose we have a cohomological spectral sequence converging to a $\mathbb{Z}$-graded abelian group $G^{*}$ with $E^{1}$ page $\left(E_{p, q}^{1}\right)_{p \in \mathbb{Z}, q \in \mathbb{Z} \text {. Define }}$

$$
\begin{gathered}
m \equiv \sup \left\{p+q: E_{1}^{p, q} \neq 0\right\} \quad \text { and } \\
k_{p} \equiv \sup \left\{p+q: q \in \mathbb{Z}, E_{1}^{p, q} \neq 0\right\} \quad \forall p \in \mathbb{Z} .
\end{gathered}
$$

Suppose that $m$ is finite and $k_{p} \neq m-1$ for all $p \in \mathbb{Z}$. Then $G^{m} \neq 0$ and $G^{k}=0$ for all $k>m$.

Proof. Let

$$
p_{m} \equiv \inf \left\{p \in \mathbb{Z}: \exists q \in \mathbb{Z}, p+q=m, E_{1}^{p, q} \neq 0\right\}
$$


We will show that each element of $E_{j}^{p_{m}, m-p_{m}}$ can never kill or be killed by the spectral sequence differential for each $j \geq 1$. Since $k_{p_{m}-j} \neq m-1$ for all $j$, we get that $k_{p_{m}-j}<m-1$ for all $j \geq 1$. Therefore $E_{j}^{p_{m}-j, m-p_{m}+j-1}=0$ for all $j \geq 1$. Hence the differential:

$$
d_{j}^{p_{m}-j, m-p_{m}+j-1}: E_{j}^{p_{m}-j, m-p_{m}+j-1} \longrightarrow E_{j}^{p_{m}, m-p_{m}}
$$

is zero for all $j$. Also since $\left(p_{m}+j\right)+\left(m-p_{m}-j+1\right)=m+1>m$, we get that $E_{j}^{p_{m}+j, m-p_{m}-j+1}=0$ for all $j$. Therefore the differential:

$$
d_{j}^{p_{m}, m-p_{m}}: E_{j}^{p_{m}, m-p_{m}} \longrightarrow E_{j}^{p_{m}+j, m-p_{m}-j+1}
$$

is zero for all $j$. Hence $G^{m} \neq 0$. Also $G^{k}=0$ for all $k>m$ since $E_{1}^{p, q}=0$ for all $p, q \in \mathbb{Z}$ satisfying $p+q=k$.

Proof of Corollary 1.3. The numbers $\mu_{m}$ do not depend on the choice of log resolution for all $m>0$ by Lemmas 2.3 and 2.6 . Hence Corollary 1.3 follows immediately from Theorem 1.2 combined with Lemmas 2.4 and 6.1

\section{Appendix A: Gradings and Canonical Bundles}

In this section we will develop tools so that we can construct gradings (See Definition 3.2) and relate them to other kinds of topological information. In this paper we will only need to study gradings up to isotopy which will be defined now. We will first give a definition of a grading for any principal $G$ bundle and then relate it to gradings of $(E, \Omega)$. Throughout this section, $G$ will be a Lie group, $\widetilde{G}$ its universal cover and $p: W \longrightarrow B$ will be a principal $G$ bundle. Also $\pi: E \longrightarrow V$, will be a symplectic vector bundle with symplectic form $\Omega$ whose fibers have dimension $2 n$.

Definition 7.1. A grading of $p$ consists of a principal $\widetilde{G}$ bundle $\widetilde{p}: \widetilde{W} \longrightarrow B$ along with a $G$ bundle isomorphism

$$
\iota: \widetilde{W} \times_{\widetilde{G}} G \cong W
$$

Note that a grading of $(E, \Omega)$ is equivalent to a grading of the principal $S p(2 n)$ bundle $\operatorname{Fr}(E)$. Let

$$
\iota_{j}: \widetilde{W}_{j} \times \widetilde{G} G \cong W, \quad j=0,1
$$

be gradings of $p$. An isotopy between these two gradings consists of a $\widetilde{G}$-bundle isomorphism

$$
\Psi: \widetilde{W}_{0} \longrightarrow \widetilde{W}_{1}
$$

together with a smooth family of $G$ bundle isomorphisms:

$$
\check{\iota}_{t}: \widetilde{W}_{0} \times \widetilde{G}_{\overparen{G}} \cong W
$$

joining $\iota_{0}$ and $\iota_{1} \circ \check{\Psi}$ where $\check{\Psi}: \widetilde{W}_{0} \times_{\widetilde{G}} G \longrightarrow \widetilde{W}_{1} \times_{\widetilde{G}} G$ is the natural isomorphism induced by $\Psi$. An isotopy between two gradings of $(E, \Omega)$ is an isotopy between the corresponding gradings on the principal $S p(2 n)$ bundle $\operatorname{Fr}(E)$. We can define isotopies of gradings of symplectic manifolds and contact manifolds in a similar way.

Definition 7.2. Let

$$
\iota: \widetilde{W} \times_{\widetilde{G}} G \cong W
$$

be a grading of $p$. The associated covering map of this grading is the natural map:

$$
\widetilde{W} \longrightarrow \widetilde{W} \times \widetilde{G} G \stackrel{\iota}{\longrightarrow} W .
$$


The following lemma gives a topological characterization of gradings. For simplicity we will assume that the base $B$ is connected. Let $\star \in W$ be a choice of basepoint.

Lemma 7.3. Let $N_{W}$ be the set of normal subgroups $A \triangleleft \pi_{1}(W, \star)$ so that

$$
p_{*}: \pi_{1}(W, \star) \longrightarrow \pi_{1}(B, p(\star))
$$

restricted to $N_{W}$ is an isomorphism. Let $G r_{W}$ be the set of isotopy classes of gradings of $W$. Then the map $Q_{W}: G r_{W} \longrightarrow N_{W}$ sending a grading to

$$
\operatorname{Im}\left(P_{*}\right) \subset \pi_{1}(W, \star)
$$

is an isomorphism where $P$ is the associated covering map of this grading.

Proof. We will first show that the map $Q_{W}$ is well defined by showing that the image (7.1) is a normal subgroup. Let

$$
\iota: \widetilde{W} \times_{\widetilde{G}} G \cong W
$$

be a grading of $p$. The covering map $P$ of such a grading is isomorphic (using the map $\iota$ ) to the natural map

$$
\widetilde{W} \longrightarrow \widetilde{W} \times \widetilde{G} G
$$

The deck transformations of this map are equal to $\operatorname{ker}(\widetilde{G} \longrightarrow G)$ and these act transitively. Hence the image (7.1) is a normal subgroup. Combining this with the fact that the fibers of the natural fibration $\widetilde{W} \longrightarrow B$ are simply connected, the image (7.1) is contained in $N_{W}$ and hence the map $Q_{W}$ is well defined.

We will now construct an inverse to $Q_{W}$. Let $N \triangleleft \pi_{1}(W, \star)$ be an element of $N_{W}$. Let

$$
P: \widetilde{W} \longrightarrow W
$$

be a covering map with a choice of basepoint $\widetilde{\star} \in \widetilde{W}$ mapping to $\star$ so that the map

$$
P_{*}: \pi_{1}(\widetilde{W}, \widetilde{\star}) \longrightarrow \pi_{1}(W, \star)
$$

has image equal to $N$. Let $F$ be a fiber of $p$. Let $\iota_{F}: F \hookrightarrow W$ be the natural inclusion map. Since $\left.P_{*}\right|_{P_{*}^{-1}(N)}$ is injective, we get that $\left(\iota_{F}\right)_{*}^{-1}\left(P_{*}^{-1}(N)\right)=\{i d\}$ and hence $\left.P\right|_{P^{-1}(F)}$ : $P^{-1}(F) \longrightarrow F$ is the universal covering map. This implies that each fiber has a natural $\widetilde{G}$ action and hence

$$
p \circ P: \widetilde{W} \longrightarrow B
$$

is a $\widetilde{G}$ bundle with a natural isomorphism

$$
\widetilde{W} \times_{\widetilde{G}} W \cong W .
$$

We define $Q_{W}^{-1}(N)$ to be the above grading. This is an inverse to $Q_{W}$.

We have the following immediate corollary of Lemma 7.3 ,

Corollary 7.4. Suppose that $p_{j}: W_{j} \longrightarrow B$ is a principal $G_{j}$ bundle for some Lie group $G_{j}$ for $j=1,2$. Let $\Phi: W_{1} \longrightarrow W_{2}$ be a map of fiber bundles so that the induced map on the fibers is a fundamental group isomorphism. Then the map $\Phi_{*}: N_{W_{1}} \longrightarrow N_{W_{2}}$ induces a natural bijection between isotopy classes of gradings on $W_{1}$ and isotopy classes of gradings on $W_{2}$.

Here the sets $N_{W_{1}}$ and $N_{W_{2}}$ in this corollary are defined as in Lemma 7.3 . 
Lemma 7.5. Let $\pi_{K}: K \longrightarrow B$ be a principal $U(1)$ bundle. Then there is a natural $1-$ 1 correspondence between homotopy classes of trivializations of $\pi_{K}$ and isotopy classes of gradings of $\pi_{K}$.

Proof. There is a $1-1$ correspondence between trivializations $\Phi: K \longrightarrow B \times U(1)$ of $K$ up to homotopy and sections of $\pi_{K}$ up to homotopy given by the map sending $\Phi$ to the section whose image is $\Phi^{-1}(1)$. Hence all we need to do is construct a natural $1-1$ correspondence between sections up to homotopy and isotopy classes of gradings. Let $S$ be the set of sections up to homotopy and $G r_{K}$ the set of isotopy classes of gradings. By Lemma 7.3, it is sufficient for us to construct a bijection between $S$ and $N_{K}$. We define

$$
\Psi: S \longrightarrow N_{K}, \quad \Psi(s)=\operatorname{Im}\left(s_{*}: \pi_{1}(B, \star) \longrightarrow \pi_{1}(K, s(\star))\right) .
$$

The inverse of this map is constructed as follows: we start with a normal subgroup $N \in N_{K}$. This gives us a grading

$$
\iota: \widetilde{K} \times_{\mathbb{R}} U(1) \cong K
$$

by Lemma $\widetilde{7.3}$ since $\widetilde{U(1)}=\mathbb{R}$. Since the fibers of $\widetilde{K}$ are contractible, there is a smooth section $\widetilde{s}: B \longrightarrow \widetilde{K}$ by [Pal66, Theorem 9] combined with the Steenrod approximation theorem [Ste99, Section 6.7, Main Theorem]. The composition $s \equiv P_{K} \circ \widetilde{s}$ where $P_{K}: \widetilde{K} \longrightarrow K$ is the associated covering map is then a smooth section of $K$. We then define $\Psi^{-1}(N) \equiv s$. This is the inverse of $\Psi$.

We will now focus on the principal $S p(2 n)$ bundle $\operatorname{Fr}(E)$.

Definition 7.6. Let $J$ be a complex structure on $E$ compatible with $\Omega$. The frame bundle $\operatorname{Fr}(E, \Omega, J)$ of the unitary bundle $(E, \Omega, J)$ is the principal $U(n)$-bundle whose fiber over $v \in V$ is the space of unitary bases $e_{1}, \cdots, e_{n}$ of $\left.(E, \Omega, J)\right|_{v}$. The anti-canonical bundle $\kappa_{J}^{*}$ of $(E, J)$ is the highest exterior power of the complex vector bundle $(E, J)$. The associated $U(1)$-bundle $\kappa_{\Omega, J}^{*} \subset \kappa_{J}^{*}$ of $\kappa_{J}^{*}$ has a fiber at $v \in V$ equal to the subset of elements $e_{1} \wedge \cdots \wedge e_{n}$ where $e_{1}, \cdots, e_{n}$ is a unitary basis for $\left.(E, \Omega, J)\right|_{v}$. Therefore we have a natural map $\operatorname{det}_{\Omega, J}$ : $\operatorname{Fr}(E, \Omega, J) \longrightarrow \kappa_{\Omega, J}$.

The canonical bundle $\kappa_{J}$ of $(E, J)$ is the dual of $\kappa_{J}^{*}$ (or equivalently the anti-canonical bundle of the dual bundle of $(E, J))$. In a similar way, we can define the (anti-)canonical bundle of a symplectic manifold with a choice of compatible almost complex structure, or of a contact manifold $\left(C, \xi_{C}\right)$ with a choice of compatible contact form $\alpha$ and a $\left.d \alpha\right|_{\xi_{C}}$-compatible almost complex structure $J$ on $\xi_{C}$.

Definition 7.7. Let $J$ be an $\Omega$-compatible complex structure on $E$. Let $\iota_{J}: F r(E, \Omega, J) \longrightarrow$ $\operatorname{Fr}(E, \Omega)$ be the natural inclusion map. By [MS04, Proposition 2.22, 2.23], the natural maps $\operatorname{det}_{\Omega, J}$ and $\iota_{J}$ above are bundle maps whose restriction each fiber is a fundamental group isomorphism. Hence by Corollary 7.4 and Lemma 7.5 there is a natural $1-1$ correspondence between isotopy classes of gradings of $(E, \Omega)$ and homotopy classes of trivializations of $\kappa_{\Omega, J}^{*}$. Combining this with the fact that there is a natural $1-1$ correspondence homotopy classes of trivializations of $\kappa_{\Omega, J}^{*}$ and homotopy classes of trivializations of $\kappa_{J}^{*}$ and hence of trivializations of the canonical bundle $\kappa_{J}$, we get a natural $1-1$ correspondence:

$$
G r:\left\{\text { Trivializations of } \kappa_{J}\right\} / \text { homotopy } \stackrel{1-1}{\longrightarrow}\{\text { Gradings of }(E, \Omega)\} / \text { isotopy. }
$$

Given a trivialization $\Phi$ of $\kappa_{J}$ we will call the grading $\operatorname{Gr}(\Phi)$ the grading associated to $\Phi$. Given a grading $g$ of $(E, \Omega)$, we will call $G r^{-1}(g)$ the trivialization associated to this grading. 
The above discussion enables us to compute the Conley-Zehnder index of a fixed point of a graded symplectomorphism in some nice cases. Let $\phi: M \longrightarrow M$ be a graded exact symplectomorphism of a Liouville domain $\left(M, \theta_{M}\right)$. Let $V$ be the unique vector field on the mapping torus $T_{\phi}$ from Definition 3.13 given by the lift of the vector field $\frac{d}{d t}$ on $\mathbb{R} / \mathbb{Z}$ satisfying $\iota_{V} d \alpha_{T_{\phi}}=0$. Let $\phi_{t}^{V}$ be the time $t$ flow of $V$. Let $x$ be a fixed point of $\phi$. Suppose that there is a compatible complex structure $J$ on the vertical tangent bundle $\left(T^{\mathrm{ver}} T_{\phi} \equiv \operatorname{ker}\left(D \pi_{T_{\phi}}\right), d \alpha_{T_{\phi}}\right)$ so that $D \phi_{t}^{V}$ restricted to $\left.T^{\mathrm{ver}} T_{\phi}\right|_{x}$ is $J$-holomorphic for all $t \in[0,1]$. Then $\phi_{t}^{V}$ lifts to a map

$$
\widetilde{\phi}_{t}:\left.\left.\kappa_{J}\right|_{x} \longrightarrow \kappa_{J}\right|_{\phi_{t}^{V}(x)}, \quad \widetilde{\phi}_{t}\left(\wedge_{i=1}^{n} e_{i}^{*}\right)=\wedge_{i=1}^{n}\left(\phi_{t}^{*}\right)^{-1} e_{i}^{*}, \quad \forall e_{1}^{*}, \cdots, e_{n}^{*} \in T_{x}^{*} M .
$$

Since $\phi$ is graded, we see by Definition 3.13 that there is a natural grading on the vertical tangent bundle. Therefore by Definition 7.7 , there is a natural trivialization $\Phi: \kappa_{J} \longrightarrow T_{\phi} \times \mathbb{C}$ of $\kappa_{J}$ associated to this grading. Let $\Phi_{2}: \kappa_{J} \longrightarrow \mathbb{C}$ be the composition of $\Phi$ with the natural projection map to $\mathbb{C}$.

Lemma 7.8. Let $x \in M=\pi_{T_{\phi}}^{-1}(0)$ be a fixed point of $\phi$ and suppose that $\left.D \phi\right|_{x}: T_{x} M \longrightarrow$ $T_{x} M$ is the identity map. Then $C Z(\phi, x)$ is equal to minus 2 times the winding number of the map:

$$
w: \mathbb{R} / \mathbb{Z} \longrightarrow \mathbb{C}^{*}=\operatorname{Aut}(\mathbb{C}, \mathbb{C}), \quad t \longrightarrow \Phi_{2} \circ \widetilde{\phi}_{t} \circ\left(\left.\Phi_{2}\right|_{\left.\kappa_{J}\right|_{x}}\right)^{-1} .
$$

Proof. Let $\gamma: \mathbb{R} / m \mathbb{Z} \longrightarrow T_{\phi}$ be the $m$-periodic orbit of $V$ whose initial point is $x$. Then there is a unique (up to homotopy) unitary trivialization $T$ of $\gamma^{*} T^{\mathrm{ver}} T_{\phi}$ so that $\Phi$ is equal to the highest wedge power of $T$. Because of the correspondence (7.2), we can also ensure that $T$ maps the grading on $\gamma^{*} T^{\text {ver }} T_{\phi}$ (given by pulling back the grading on $T^{\text {ver }} T_{\phi}$ via $\gamma$ ) to the trivial grading on $(\mathbb{R} / m \mathbb{Z}) \times \mathbb{C}^{n+1}$ (maybe after changing the grading to an isotopic one).

Under this trivialization, the flow of $V$ corresponds to a smooth family of Hermitian matrices $\left(A_{t}\right)_{t \in[0,1]}$ and the degree of $w$ is -1 times the winding number of $t \longrightarrow \operatorname{det}_{\mathbb{C}}\left(A_{t}\right)$. Using the correspondence (7.2) and the trivialization $T$, such a family of matrices corresponds to a point in the universal cover $\left.\widetilde{F r}(T M)\right|_{x}$ of $\left.\operatorname{Fr}(T M)\right|_{x}$. Hence the Conley-Zehnder index of $\left(A_{t}\right)_{t \in[0,1]}$ is equal to $C Z(\phi, x)$. Since $A_{t}$ are unitary matrices, we get that $C Z\left(\left(A_{t}\right)_{t \in[0,1]}\right)$ is equal to twice the winding number of $t \longrightarrow \operatorname{det}_{\mathbb{C}}\left(A_{t}\right)$. Hence $C Z(\phi, x)$ is -2 times the winding number of $w$.

\section{Appendix B: Contactomorphisms of Mapping Tori and Floer Cohomology}

The aim of this section is to show that Property (HF2) holds. Here is a statement of this property:

Suppose that $\left(M_{1}, \theta_{M_{1}}, \phi_{1}\right),\left(M_{2}, \theta_{M_{2}}, \phi_{2}\right)$ are graded abstract contact open books so that the graded contact pairs associated to them are graded contactomorphic. Then $\operatorname{HF}^{*}\left(\phi_{0},+\right)=$ $H F^{*}\left(\phi_{1},+\right)$.

We will prove this by using an intermediate Floer cohomology group called $S^{1}$-equivariant Hamiltonian Floer cohomology on a certain mapping cylinder of our symplectomorphism.

Definition 8.1. Let $\left(M, \theta_{M}, \phi\right)$ be an abstract contact open book. Let $\check{\phi}$ be a small positive slope perturbation of $\phi$. The mapping cylinder of $\check{\phi}$ is a triple $\left(W_{\check{\phi}}, \pi_{\check{\phi}}, \theta_{\check{\phi}}\right)$ where

(1) $W_{\check{\phi}} \equiv(\mathbb{R} \times \mathbb{R} \times M) / \mathbb{Z}$ where the $\mathbb{Z}$ action on $(\mathbb{R} \times \mathbb{R} \times M)$ has the property that $1 \in \mathbb{Z}$ sends $(s, t, x)$ to $(s, t-1, \check{\phi}(x))$,

(2) $\pi_{\check{\phi}}: W_{\check{\phi}} \longrightarrow \mathbb{R} \times(\mathbb{R} / \mathbb{Z})$ sends $(s, t, x)$ to $(s, t) \in \mathbb{R} \times \mathbb{R} / \mathbb{Z}$ and

(3) $\theta_{\check{\phi}}=s d t+\kappa \theta_{M}+\kappa d\left(\rho(t) F_{\check{\phi}}\right)$ where 
- $F_{\check{\phi}}: M \longrightarrow \mathbb{R}$ is a smooth function with support in the interior of $M$ satisfying $(\check{\phi})^{*} \theta_{M}=\theta_{M}+d F_{\check{\phi}}$,

- $\rho:[0,1] \longrightarrow[0,1]$ is a a smooth function equal to 0 near 0 and 1 near 1 and

- $\kappa>0$ is a constant small enough to ensure that $d \theta_{\breve{\phi}}$ is symplectic.

Let $r_{M}:(0,1] \times \partial M \longrightarrow(0,1]$ be the cylindrical coordinate on $M$. Let $\delta_{\check{\phi}}>0$ be small enough so that the symplectomorphism $\check{\phi}$ is equal to the time 1 flow of $\delta r_{M}$ inside $\left(1-\delta_{\check{\phi}}, 1\right] \times \partial M$ for some $\delta>0$. Let $\phi_{t}^{\delta r_{M}}:\left(1-\delta_{\check{\phi}}, 1\right] \times \partial M \longrightarrow\left(1-\delta_{\check{\phi}}, 1\right] \times \partial M$ be the time $t$ flow of $\delta r_{M}$. Then we have a natural embedding:

$$
\iota_{\check{\phi}}: C_{\check{\phi}} \equiv\left(\mathbb{R} \times(\mathbb{R} / \mathbb{Z}) \times\left(1-\delta_{\check{\phi}}, 1\right] \times \partial M\right) \hookrightarrow W_{\check{\phi}}, \quad \iota_{\check{\phi}}\left(s, t, r_{M}, y\right) \equiv\left(s, t,\left(\phi_{-t}^{\delta r_{M}}\left(r_{M}, y\right)\right)\right.
$$

called the vertical cylindrical end of $W_{\check{\phi}}$. The coordinate

$$
r_{\check{\phi}}: C_{\check{\phi}} \longrightarrow\left(1-\delta_{\check{\phi}}, 1\right], \quad r_{\check{\phi}}(s, t, x) \equiv r_{M}(x)
$$

is called the vertical cylindrical coordinate. A grading on a mapping cylinder $\left(W_{\check{\phi}}, \pi_{\check{\phi}}, \theta_{\check{\phi}}\right)$ is a grading on the symplectic manifold $\left(W_{\check{\phi}}, d \theta_{\check{\phi}}\right)$.

Two mapping cylinders $\left(W_{\check{\phi}_{1}}, \pi_{\check{\phi}_{1}}, \theta_{\check{\phi}_{1}}\right),\left(W_{\check{\phi}_{2}}, \pi_{\check{\phi}_{2}}, \theta_{\check{\phi}_{2}}\right)$ are isomorphic if there is a diffeomorphism $\Phi: W_{\check{\phi}_{1}} \longrightarrow W_{\check{\phi}_{2}}$ and a constant $\delta>0$ so that

- $\Phi^{*} \theta_{\check{\phi}_{2}}=\theta_{\check{\phi}_{1}}+d q$ for some $q: W_{\check{\phi}_{1}} \longrightarrow \mathbb{R}$ where $q$ has support in $W_{\check{\phi}_{1}}-\left\{r_{\check{\phi}_{1}} \geq 1-\delta\right\}$ and

- $\left.\pi_{\check{\phi}_{1}}\right|_{\left\{r_{\check{\phi}_{1}} \geq 1-\delta\right\}}=\left.\left(\pi_{\check{\phi}_{2}} \circ \Phi\right)\right|_{\left\{r_{\check{\phi}_{1}} \geq 1-\delta\right\}}$.

They are graded isomorphic if, in addition, $\Phi$ is a graded symplectomorphism from $\left(W_{\check{\phi}_{1}}, d \theta_{\check{\phi}_{1}}\right)$ to $\left(W_{\check{\phi}_{2}}, d \theta_{\check{\phi}_{2}}\right)$.

Note that the definition above has many similarities with the definition of the mapping torus from Definition 3.13. Also if we define the mapping torus

$$
\pi_{T_{\check{\phi}}}: T_{\check{\phi}} \longrightarrow \mathbb{R} / \mathbb{Z}, \alpha_{T_{\check{\phi}}}
$$

of our positive slope perturbation $\check{\phi}$ in exactly the same way as in Definition 3.13, then $W_{\check{\phi}}=\mathbb{R} \times T_{\check{\phi}}, \theta_{\check{\phi}}=(s-\kappa) d t+\frac{\kappa}{C} \alpha_{\check{\phi}}$ for some $C>0$ and $\pi_{\check{\phi}}=\operatorname{id}_{\mathbb{R}} \times \pi_{T_{\check{\phi}}}$. The following calculation will be useful later on. If we have a Hamiltonian $H$ equal to $\pi_{\phi}^{*} K$ for some $K: \mathbb{R} \times(\mathbb{R} / \mathbb{Z}) \longrightarrow \mathbb{R}$ then $X_{H}$ is equal to the horizontal lift of $X_{K}^{d s \wedge d t}$ with respect to the symplectic connection associated to $d \theta_{\check{\phi}}$.

Lemma 8.2. Let

$$
\left(B_{1} \subset C_{1}, \xi_{1}, \tau_{1}\right), \quad\left(B_{2} \subset C_{2}, \xi_{2}, \tau_{2}\right)
$$

be the (graded) contact pairs associated to the (graded) abstract contact open books $\left(M_{1}, \theta_{M_{1}}, \phi_{1}\right)$, $\left(M_{2}, \theta_{M_{2}}, \phi_{2}\right)$ respectively. Let $\check{\phi}_{1}$ be a small positive slope perturbation of $\phi_{1}$. If the above contact pairs are (graded) contactomorphic then $\left(M_{2}, \theta_{M_{2}}, \phi_{2}\right)$ is (graded) isotopic to an abstract contact open book $\left(M_{3}, \theta_{M_{3}}, \phi_{M_{3}}\right)$ so that the mapping cylinders

$$
\left(W_{\check{\phi}_{1}}, \pi_{\check{\phi}_{1}}, \theta_{\check{\phi}_{1}}\right), \quad\left(W_{\check{\phi}_{3}}, \pi_{\check{\phi}_{3}}, \theta_{\check{\phi}_{3}}\right)
$$

are (graded) isomorphic where $\check{\phi}_{3}$ is a small positive slope perturbation of $\phi_{3}$.

Proof. Since the corresponding open books are contactomorphic, and the boundary $\partial M_{j}$ is contactomorphic to the binding of $O B D\left(M_{j}, \theta_{M_{j}}, \phi_{j}\right)$ for $j=1,2$, we get that $\partial M_{1}$ is contactomorphic to $\partial M_{2}$. Hence there is a diffeomorphism $\Psi: \partial M_{2} \longrightarrow \partial M_{1}$ so that $\Psi^{*} \alpha_{1}=$ $f \alpha_{2}$ where $\alpha_{j}=\left.\theta_{M_{j}}\right|_{\partial M_{j}}$ for $j=1,2$ and $f: \partial M_{2} \longrightarrow(0, \infty)$. After multiplying $\theta_{2}$ by 
a positive constant, we can assume that $f>1$. Choose $\delta>0$ small enough so that the subset of the cylindrical end $(1-\delta, 1] \times \partial M_{2} \subset M_{2}$ is disjoint from the support of $\phi_{2}$ and let $r_{M_{2}}:(1-\delta, 1] \times \partial M_{2} \longrightarrow(1-\delta, 1]$ be the associated cylindrical coordinate. Let $\rho$ : $(1-\delta, 1] \longrightarrow[0,1]$ be a smooth function with non-negative derivative so that it is equal to 0 near $1-\delta$ and 1 near 1 . Let $F_{t}: M_{2} \longrightarrow \mathbb{R}, t \in[0,1]$ be a smooth family of functions equal to $1+t \rho\left(r_{M_{2}}\right)(f-1)$ inside $(1-\delta] \times \partial M_{2}$ and 1 otherwise. Then $\left(M_{2}, F_{t} \theta_{M_{2}}, \phi_{2}\right)$ is an isotopy of abstract contact open books. Define $\left(M_{3}, \theta_{M_{3}}, \phi_{3}\right) \equiv\left(M_{2}, F_{1} \theta_{M_{2}}, \phi_{2}\right)$. Hence there is a diffeomorphism $\check{\Psi}: \partial M_{3} \longrightarrow \partial M_{1}$ so that $(\check{\Psi})^{*} \alpha_{M_{1}}=\alpha_{M_{3}}$ where $\left.\alpha_{M_{3}} \equiv \theta_{M_{3}}\right|_{\partial M_{3}}$.

Choose a small positive slope perturbation $\check{\phi}_{3}$ of $\phi_{3}$ so that $\left.\check{\phi}_{3}\right|_{(1-\check{\delta}, 1] \times \partial M_{3}}$ is equal to $\left.\left(\operatorname{id}_{(1-\check{\delta}, 1]} \times \check{\Psi}\right)^{*} \check{\phi}_{1}\right|_{(1-\check{\delta}, 1] \times \partial M_{1}}$ for some $\check{\delta}>0$ smaller than $\delta$. Let $\left(T_{\phi_{j}}, \pi_{T_{\phi_{j}}}, \alpha_{\phi_{j}}\right)$ be the mapping torus of $\phi_{j}$ and let $\left(T_{\check{\phi}_{j}}, \pi_{T_{\check{\phi}_{j}}}, \alpha_{\check{\phi}_{j}}\right)$ be the mapping torus of $\check{\phi}_{j}$ for $j=1,3$. Let $\left(B_{3} \subset C_{3}, \xi_{3}, \tau_{3}\right)$ be the contact pair associated to $\left(M_{3}, \theta_{M_{3}}, \phi_{3}\right)$. By lemma 5.34, we get that the contact pair associated to $\left(M_{3}, \theta_{M_{3}}, \phi_{3}\right)$ is contactomorphic to the contact pair associated to $\left(M_{2}, \theta_{M_{3}}, \phi_{2}\right)$ which in turn is contactomorphic to the contact pair associated to $\left(M_{1}, \theta_{M_{1}}, \phi_{1}\right)$. There is a contactomorphism $Q: T_{\phi_{3}} \longrightarrow T_{\phi_{1}}$ so that $\left.Q\right|_{B_{3}}: B_{3} \longrightarrow B_{1}$ is equal to $\check{\Psi}$ under the identification $B_{i}=M_{i}$ for $i=1,3$ and so that $\pi_{T_{\phi_{3}}}=\pi_{T_{\phi_{1}}} \circ Q$ near $\partial T_{\phi_{3}}$. Hence we can find a contactomorphism $\check{Q}: T_{\check{\phi}_{3}} \longrightarrow T_{\check{\phi}_{1}}$ satisfying $(\check{Q})^{*} \alpha_{\check{\phi}_{1}}=\alpha_{\check{\phi}_{3}}$ near $\partial T_{\check{\phi}_{3}}$ and so that $\pi_{T_{\check{\phi}_{3}}}=\pi_{T_{\check{\phi}_{1}}} \circ \check{Q}$ near $\partial T_{\check{\phi}_{3}}$.

Since $W_{\check{\phi}_{j}}$ is naturally diffeomorphic to $\mathbb{R} \times T_{\check{\phi}_{j}}$ for $j=1,3$ we can define

$$
W: W_{\check{\phi}_{3}} \longrightarrow W_{\check{\phi}_{1}}, \quad W \equiv\left(\mathrm{id}_{\mathbb{R}}, \check{Q}\right)
$$

Now $W^{*} \theta_{\check{\phi}_{3}}=\theta_{\check{\phi}_{1}}$ outside a subset $K \subset W_{\check{\phi}_{3}}$ whose intersection with each fiber of $\pi_{\check{\phi}_{3}}$ is compact. Since $\theta_{\check{\phi}_{3}}$ and $W^{*} \theta_{\check{\phi}_{1}}$ scale at most linearly in $C^{1}$ norm as we translate in the $s$ coordinate direction, we can use a Moser argument applied to $\tau \theta_{\check{\phi}_{3}}+(1-\tau) W^{*} \theta_{\check{\phi}_{1}}, \tau \in[0,1]$ giving us our isomorphism.

Lemma 8.3. Suppose that $\left(M_{1}, \theta_{M_{1}}, \phi_{1}\right),\left(M_{2}, \theta_{M_{2}}, \phi_{2}\right)$ are isotopic abstract contact open books. Then $H F^{*}\left(\phi_{1},+\right)=H F^{*}\left(\phi_{2},+\right)$.

Proof. Since the above abstract contact open books are isotopic, we can assume that $M_{1}=M_{2}$ and that there is a smooth family of Liouville forms $\left(\theta_{s}\right)_{s \in[0,1]}$ so that $\theta_{0}=\theta_{M_{1}}$ and $\theta_{1}=\theta_{M_{2}}$. Also there is a smooth family of exact symplectomorphisms $\psi_{s}: M_{1} \longrightarrow M_{1}, s \in[0,1]$ with respect to $\theta_{s}$ with support in a fixed compact set joining $\phi_{1}$ and $\phi_{2}$. Let $r_{s}$ be the cylindrical coordinate for $\left(M_{1}, \theta_{s}\right)$ and choose $\delta>0$ small enough so that $\left\{r_{s} \geq 1-\delta\right\}$ is disjoint from the support of $\psi_{s}$ for all $s \in[0,1]$. By pulling back $r_{s}$ and $\theta_{s}$ and $\psi_{s}$ by a smooth family of diffeomorphisms starting at the identity and parameterized by $s \in[0,1]$, we can assume that $r_{s}=r_{0}$ inside the region $\left\{r_{0} \geq 1-\delta\right\}=(1-\delta, 1] \times \partial M_{1}$. By Gray's stability theorem, we can also assume that $\theta_{s}=r_{0} f_{s} \alpha$ inside $\left\{r_{0} \geq 1-\delta\right\}=(1-\delta, 1] \times \partial M_{1}$ for some contact form $\alpha$ on $\partial M_{1}$ and some smooth family of functions $f_{s}: \partial M_{1} \longrightarrow(0, \infty), s \in[0,1]$.

Now choose a smooth family of functions $g_{s}:(1-\delta, 1] \times \partial M_{1} \longrightarrow(0, \infty), s \in[0,1]$ so that $\frac{\partial g_{s}}{\partial r_{0}}>0, g_{s}$ is equal to $f_{s}$ inside $(1-\delta, 1-\delta / 2] \times \partial M_{1}$, and $g_{s}=C f_{0}$ inside $(1-\delta / 4,1] \times \partial M_{1}$ for some large constant $C>0$ and for all $t \in[0,1]$. Define $\check{\theta}_{s}$ to be equal to $\theta_{s}$ outside $(1-\delta, 1] \times \partial M_{1}$ and equal to $r_{0} g_{s} \alpha$ inside this region. Then $\left(M_{1}, \check{\theta}_{s}\right), s \in[0,1]$ is a smooth family of Liouville domains so that $\check{\theta}_{s}=\theta_{s}$ inside $(1-\delta, 1-\delta / 2] \times \partial M_{1}$ and $\check{\theta}_{s}$ is independent of $s$ near $\partial M_{1}$. 
Let $\left(K_{s, t}\right)_{(s, t) \in[0,1]^{2}}$ be a smooth family of almost complex structures compatible with $d \theta_{s}$ so that $K_{s, t}$ is cylindrical inside $(1-\delta] \times M_{1}$ with respect to $\theta_{s}$ for all $(s, t) \in[0,1]^{2}$. Choose a smooth family of almost complex structures $\left(J_{s, t}\right)_{(s, t) \in[0,1]^{2}}$ compatible with $d \check{\theta}_{s}$ equal to $K_{s, t}$ outside $(1-\delta / 2,1] \times \partial M_{1}$ and equal to $K_{0, t}$ inside $(1-\delta / 4,1] \times \partial M_{1}$. Let $\check{\psi}_{s}$ be a smooth family of exact symplectomorphisms with respect to $\check{\theta}_{s}$ which are small positive slope perturbations of $\psi_{s}$ so that $\breve{\psi}_{s}$ is the time 1 flow of $\eta r_{s}$ inside $(1-\delta, 1] \times \partial M_{1}$ for some very small $\eta>0$ (so that there are no fixed points of this symplectomorphism in this region). Let $\widehat{\psi}_{s}$ be a smooth family of positive slope perturbations of $\psi_{s}$ with respect to $\theta_{s}$ which are equal to the time 1 flow of $\eta r_{s}$ inside $(1-\delta, 1] \times \partial M_{1}$ with respect to the symplectic structure $d \theta_{s}$. We assume that $\eta$ is small enough so that $\widehat{\psi}_{s}$ has no fixed points inside $(1-\delta, 1] \times \partial M_{1}$ for all $s \in[0,1]$.

Since

- $\check{\psi}_{s}, \widehat{\psi}_{s}$ is the time 1 flow of a linear Hamiltonian inside $(1-\delta, 1-\delta / 2) \times \partial M_{1}$,

- $J_{s, t}$ and $K_{s, t}$ are cylindrical inside this region and

- $\left(\check{\psi}_{s},\left(J_{s, t}\right)_{t \in[0,1]}\right),\left(\widehat{\psi}_{s},\left(K_{s, t}\right)_{t \in[0,1]}\right)$ are equal outside $(1-\delta, 1] \times \partial M_{1}$ and

- $\check{\psi}_{s}$ and $\widehat{\psi}_{s}$ has no fixed points inside $(1-\delta, 1] \times \partial M_{1}$

then a maximum principle ([AS10, Lemma 7.2]) tells us that

$$
H F^{*}\left(\check{\psi}_{s},\left(J_{s, t}\right)_{t \in[0,1]}\right)=H F^{*}\left(\widehat{\psi}_{s},\left(K_{t}\right)_{t \in[0,1]}\right)
$$

for all $s \in[0,1]$.

Since $\check{\theta}_{s}$ is independent of $s$ near $\partial M_{1}$, we can assume, by a Moser argument, that $\check{\theta}_{s}=$ $\theta_{0}+\beta_{s}$ for some smooth family of compactly supported closed 1 -forms $\beta_{s}, s \in[0,1]$. Then by [Ulj14, Theorem 2.34], we get that $H F^{*}\left(\breve{\psi}_{s},\left(J_{s, t}\right)_{t \in[0,1]}\right)$ is independent of $s \in[0,1]$. Hence $H F^{*}\left(\widehat{\psi}_{s},\left(K_{s}\right)_{t \in[0,1]}\right)$ is independent of $s$ by Equation (8.2) which implies that $H F^{*}\left(\phi_{1},+\right)=$ $H F^{*}\left(\phi_{2},+\right)$.

Definition 8.4. Let $\left(W_{\check{\phi}}, \pi_{\check{\phi}}, \theta_{\check{\phi}}\right)$ be a mapping cylinder. An almost complex structure $J$ on $W_{\check{\phi}}$ is strictly compatible with $\left(W_{\check{\phi}}, \pi_{\check{\phi}}, \theta_{\check{\phi}}\right)$ if

(1) $J$ is compatible with $d \theta_{\check{\phi}}$,

(2) $\pi_{\check{\phi}}: W_{\check{\phi}} \longrightarrow \mathbb{R} \times \mathbb{R} / \mathbb{Z}$ is $\left(J, j\right.$ )-holomorphic (I.e. $D \pi_{\check{\phi}} \circ J=j \circ D \pi_{\check{\phi}}$ ) where $j$ is the complex structure sending $\frac{\partial}{\partial s}$ to $\frac{\partial}{\partial t}$ where $(s, t)$ parameterizes $\mathbb{R} \times \mathbb{R} / \mathbb{Z}$,

(3) the restriction of $J$ to the cylindrical end $C_{\breve{\phi}}$ is a product $j \oplus J_{M}$ where $J_{M}$ is a fixed cylindrical almost complex structure inside the cylindrical end $\left(1-\delta_{\check{\phi}}, 1\right] \times \partial M$ and

(4) $J$ is invariant under translations in the $s$ coordinate.

We will call $J_{M}$ the associated cylindrical almost complex structure on $M$. An almost complex structure $J$ on $W_{\check{\phi}}$ is compatible with $\left(W_{\check{\phi}}, \pi_{\check{\phi}}, \theta_{\check{\phi}}\right)$ if there is an almost complex structure $\breve{J}$ compatible with $\left(W_{\check{\phi}}, \pi_{\check{\phi}}, \theta_{\check{\phi}}\right)$ and a compact subset $K$ in the interior of $W_{\check{\phi}}$ so that $\left.J\right|_{W_{\check{\phi}}-K}=$ $\left.\check{J}\right|_{W_{\check{\phi}}-K}$.

Definition 8.5. Recall that a 1-periodic orbit of a time dependent Hamiltonian $H_{t}: W_{\check{\phi}} \longrightarrow$ $\mathbb{R}$ is a smooth map $\gamma: \mathbb{R} / \mathbb{Z} \longrightarrow M$ satisfying $\frac{d}{d t} \gamma=X_{H_{t}}^{d \theta_{\check{\phi}}}$. Since there is a natural 1-1 correspondence between 1-periodic orbits and fixed points of the corresponding Hamiltonian symplectomorphism $\phi_{1}^{H_{t}}$, we will call $\gamma$ the 1-periodic orbit associated to the fixed point $\gamma(0)$. A Hamiltonian is autonomous if it does not depend on time. An $S^{1}$-family of 1-periodic orbits of $H$ is a family $\left(\gamma_{t}\right)_{t \in \mathbb{R} / \mathbb{Z}}$ of 1 -periodic orbits where $\gamma_{t}(\check{t})=\gamma_{0}(\check{t}+t)$ for all $t, \check{t} \in \mathbb{R} / \mathbb{Z}$. 
Let $(H, J),(\check{H}, \check{J})$ be pairs consisting of autonomous Hamiltonians $H, \check{H}$ and almost complex structures $J, \check{J}$. A smooth family of pair $\left(H_{s}, J_{s}\right)_{s \in \mathbb{R}}$ joins $(H, J)$ and $(\check{H}, \check{J})$ if $\left(H_{s}, J_{s}\right)=(H, J)$ for all sufficiently negative $s$ and $\left(H_{s}, J_{s}\right)=(\check{H}, \check{J})$ for all sufficiently positive $s$.

Let $h: \mathbb{R} \longrightarrow \mathbb{R}$ be a smooth function which is bounded from below with positive derivative satisfying $h^{\prime \prime}(s)=0$ whenever $s$ is sufficiently positive and $h^{\prime}(s)<1$ for $s$ sufficiently negative. The value of $h^{\prime}(s)$ for large enough $s$ is called the slope of $h$. A Hamiltonian is strictly compatible with $\left(W_{\check{\phi}}, \pi_{\check{\phi}}, \theta_{\check{\phi}}\right)$ if it is equal to $\pi_{\check{\phi}}^{*} h(s)$ everywhere. A Hamiltonian is compatible with $\left(W_{\check{\phi}}, \pi_{\check{\phi}}, \theta_{\check{\phi}}\right)$ if it is equal to $\pi_{\check{\phi}}^{*} h(s)$ outside a compact subset of the interior of $W_{\check{\phi}}$. The slope of such a Hamiltonian is defined to be the slope of $h$.

All the 1-periodic orbits of $h(s)$ on the symplectic manifold $(\mathbb{R} \times \mathbb{R} / \mathbb{Z}, d s \wedge d t)$ wrapping around $\mathbb{R} \times \mathbb{R} / \mathbb{Z}$ once come in $S^{1}$ families in the region $h^{\prime}(s)=1$ and for the unique $s$ satisfying $h^{\prime}(s)=1$ we have 1-periodic orbits

$$
\gamma_{s, q}: \mathbb{R} / \mathbb{Z} \longrightarrow \mathbb{R} \times \mathbb{R} / \mathbb{Z}, \quad \gamma_{q}(t)=(s, t+q)
$$

for all $q \in[0,1)$. Also the 1-periodic orbits of $\pi_{\dot{\phi}}^{*} h(s)$ project to 1-periodic orbits of $h(s)$.

A pair $(H, J)$ is (strictly) compatible with $\left(W_{\check{\phi}}, \pi_{\check{\phi}}, \theta_{\check{\phi}}\right)$ if $H$ is a Hamiltonian (strictly) compatible with $\left(W_{\check{\phi}}, \pi_{\check{\phi}}, \theta_{\check{\phi}}\right)$ and $J$ is an almost complex structure (strictly) compatible with $\left(W_{\check{\phi}}, \pi_{\check{\phi}}, \theta_{\check{\phi}}\right)$. A smooth family $\left(H_{s}, J_{s}\right)_{s \in \mathbb{R}}$ of pairs compatible with $\left(W_{\check{\phi}}, \pi_{\check{\phi}}, \theta_{\check{\phi}}\right)$ has non-increasing slope if the slope of $H_{s}$ is greater than or equal to the slope of $H_{\breve{s}}$ for all $s \leq \check{s}$.

Definition 8.6. Let $\left(H_{\sigma}, J_{\sigma}\right)_{\sigma \in \mathbb{R}}$ be a smooth family of pairs of Hamiltonians and almost complex structures compatible with a mapping cylinder $\left(W_{\check{\phi}}, \pi_{\check{\phi}}, \theta_{\check{\phi}}\right)$. An open subset $V \subset W_{\check{\phi}}$ satisfies the maximum principal with respect to $\left(H_{\sigma}, J_{\sigma}\right)_{\sigma \in \mathbb{R}}$ if for every compact codimension 0 submanifold $\Sigma \subset \mathbb{R} \times \mathbb{R} / \mathbb{Z}$ and every smooth map $u: \Sigma \longrightarrow W_{\check{\phi}}$ satisfying

(1) $u(\partial \Sigma) \subset V$

(2) $\partial_{\sigma} u(\sigma, \tau)+J_{\sigma} \partial_{\tau} u(\sigma, \tau)=J_{\sigma} X_{H_{\sigma}}$, also satisfies $\operatorname{Im}(u) \subset V$.

We say that $\left(H_{\sigma}, J_{\sigma}\right)_{\sigma \in \mathbb{R}}$ satisfies the maximum principle if there is a sequence of relatively compact open sets $\left(V_{i}\right)_{i \in \mathbb{N}}$ whose union is $W_{\check{\phi}}$ so that $\left(H_{\sigma}, J_{\sigma}\right)_{\sigma \in \mathbb{R}}$ satisfies the maximum principle with respect to $V_{i}$ for all $i \in \mathbb{N}$.

Lemma 8.7. Let $\left(M, \theta_{M}, \phi\right)$ be an abstract contact open book and let $W \equiv\left(W_{\check{\phi}}, \pi_{\check{\phi}}, \theta_{\check{\phi}}\right)$ be a mapping cylinder of some positive slope perturbation of $\phi$. Let $\left(K_{\sigma} \equiv \pi_{\tilde{\phi}}^{*}\left(k_{\sigma}(s)\right)\right)_{\sigma \in \mathbb{R}}$ be a smooth family of Hamiltonians strictly compatible with $W$ so that $\frac{d k_{\sigma}}{d \sigma} \leq 0, \frac{d k_{\sigma}^{\prime}}{d \sigma} \leq 0$ and $\frac{d k_{\sigma}^{\prime \prime}}{d \sigma} \leq 0$. Let $Y$ be an almost complex structure strictly compatible with $W$.

Let $\delta, S>0$ and let $r_{\tilde{\phi}}$ be the vertical cylindrical coordinate of $W$. Let $\left(H_{\sigma}, J_{\sigma}\right)_{\sigma \in \mathbb{R}}$ be a smooth family of pairs compatible with $W$ which are equal to $\left(K_{\sigma}, Y\right)$ near the boundary of the set $V_{\delta, S} \equiv \pi_{\check{\phi}}^{-1}((-S, S) \times(\mathbb{R} / \mathbb{Z})) \subset W_{\check{\phi}}$ and also in the region $\left\{r_{\check{\phi}} \geq 1-\delta\right\}$. Then $V_{\delta, S}$ satisfies the maximum principal with respect to $\left(H_{\sigma}, J_{\sigma}\right)_{\sigma \in \mathbb{R}}$.

Proof. Let $u: \Sigma \longrightarrow W_{\check{\phi}}$ be as in Definition 8.6 with $V$ replaced by $V_{\delta, S}$. Let

$$
\iota_{\check{\phi}}: C_{\check{\phi}} \equiv\left(\mathbb{R} \times(\mathbb{R} / \mathbb{Z}) \times\left(1-\delta_{\check{\phi}}, 1\right] \times \partial M\right) \hookrightarrow W_{\check{\phi}}, \quad \iota_{\check{\phi}}\left(s, t, r_{M}, y\right) \equiv\left(s, t,\left(\phi_{-t}^{\delta r_{M}}\left(r_{M}, y\right)\right)\right.
$$

be the vertical cylindrical end of $W_{\check{\phi}}$. Then $r_{\check{\phi}}: C_{\check{\phi}} \longrightarrow\left(1-\delta_{\check{\phi}}, 1\right]$ is the natural projection map. Let $\left.P_{M}: C_{\check{\phi}} \longrightarrow\left(1-\delta_{\check{\phi}}, 1\right] \times \partial M\right)$ be the natural projection map. Consider the map

$$
\left.\widehat{u}: u^{-1}\left(C_{\check{\phi}}\right) \longrightarrow\left(1-\delta_{\check{\phi}}, 1\right] \times \partial M\right), \quad \widehat{u}(\sigma, \tau)(x) \equiv P_{M} \circ u(x) .
$$


Let $J_{M, \sigma, t}$ be the natural almost complex structure on $\left(1-\delta_{\check{\phi}}\right) \times \partial M$ induced by $\left.J_{\sigma}\right|_{\pi_{\tilde{\phi}}^{-1}(s, t)}$ for some $s$. Such an almost complex structure does not depend on $s$ and is cylindrical by definition. Since $J_{\sigma} X_{H_{\sigma}}$ is a multiple of $\frac{\partial}{\partial s}$, we get that $\widehat{u}$ satisfies

$$
\frac{\partial \widehat{u}}{\partial \sigma}+J_{M} \frac{\partial \widehat{u}}{\partial \tau}-J_{M} X_{\delta r_{M}}=0
$$

where $r_{M}$ is the cylindrical coordinate on $M$ and $\delta>0$ is some constant. Therefore by applying [AS10, Lemma 7.2] to $\widehat{u}$ we see that such a map cannot intersect the region $(1-$ $\delta, 1] \times \partial M)$ and hence the image of $u$ cannot intersect the region $\left\{r_{\check{\phi}} \geq 1-\delta\right\}$.

Therefore we only need to show that the image of $u$ is contained inside $\pi_{\check{\phi}}^{-1}((-S, S) \times \mathbb{R} / \mathbb{Z})$. First of all we can make $S$ very slightly smaller so that $u$ is transverse to $\pi_{\tilde{\phi}}^{-1}(\{S,-S\})$ and so that $u(\partial \Sigma) \subset V_{\delta, S}$. This implies that $\check{\Sigma} \subset \mathbb{R} \times \mathbb{R} / \mathbb{Z}$ is a smooth submanifold with boundary. Suppose for a contradiction that $\check{\Sigma}$ is nonempty. Choose $\delta_{1}>0$ small enough so that $\left(H_{\sigma}, J_{\sigma}\right)=\left(K_{\sigma}, Y_{\sigma}\right)$ inside $\pi_{\check{\phi}}^{-1}\left(\left(\left[-S-\delta_{1},-S\right] \cup\left[S, S+\delta_{1}\right]\right) \times \mathbb{R} / \mathbb{Z}\right)$ for all $\sigma \in \mathbb{R}$ and so that $u$ intersects $\pi_{\check{\phi}}^{-1}(\{s\} \times \mathbb{R} / \mathbb{Z})$ transversely for all $s \in\left[-S-\delta_{1},-S\right] \cup[S, S+$ $\left.\delta_{1}\right]$. Now let $\beta: \mathbb{R} \longrightarrow \mathbb{R}$ be a smooth function satisfying $\beta^{\prime} \geq 0,\left.\beta\right|_{\left(-S-\delta_{1} / 2, S+\delta_{1} / 2\right)}=0$, $\left.\beta^{\prime}\right|_{\left(-S-3 \delta_{1} / 4,-S-\delta_{1} / 2\right) \cup\left(S+\delta_{1} / 2, S+3 \delta_{1} / 4\right)}>0$ and $\beta$ is constant outside $\left(-S-3 \delta_{1} / 4, S+3 \delta_{1} / 4\right)$. Choose a smooth function $q_{\sigma}: \mathbb{R} \longrightarrow \mathbb{R}$ so that $\left.q_{\sigma}\right|_{\left(-S-\delta_{1} / 2, S+\delta_{1} / 2\right)}=0$ and $q_{\sigma}^{\prime}=\beta^{\prime} k_{\sigma}^{\prime}$. Then $\frac{d q_{\sigma}^{\prime}}{d \sigma} \leq 0$. Hence

$$
\begin{gathered}
\left.0<\int_{\check{\Sigma}} \beta^{\prime}(s(u))\left|\left(\pi_{\check{\phi}}\right)_{*}\left(\frac{\partial u}{\partial \sigma}\right)\right|^{2} d \sigma \wedge d \tau=\int_{\check{\Sigma}} u^{*} d(\beta(s) d t)\right)\left(\frac{\partial u}{\partial \sigma}, \frac{\partial u}{\partial \tau}-X_{H_{\sigma}}\right) d \sigma \wedge d \tau \\
\left.=\int_{\check{\Sigma}} u^{*} d(\beta(s) d t)\right)-u^{*}\left(\beta^{\prime}(s) d H_{\sigma}\right) \wedge d \tau= \\
\left.\int_{\check{\Sigma}} u^{*} d(\beta(s) d t)\right)-d\left(u^{*}\left(q_{\sigma}(s)\right)\right) \wedge d \tau+u^{*}\left(\frac{d q_{\sigma(s)}}{d \sigma}\right) d \sigma \wedge d \tau \\
\leq \int_{\check{\Sigma}} u^{*} d(\beta(s) d t)-d\left(q_{\sigma}(s(u)) d \tau\right)=\int_{\partial \check{\Sigma}} u^{*}(\beta(s) d t)-q_{\sigma}(s(u)) d \tau=0,
\end{gathered}
$$

giving us a contradiction.

Corollary 8.8. Any smooth family $\left(H_{\sigma}, J_{\sigma}\right)_{\sigma \in \mathbb{R}}$ compatible with $\left(W_{\check{\phi}}, \pi_{\check{\phi}}, \theta_{\check{\phi}}\right)$ with non-increasing slope satisfies the maximum principle.

Definition 8.9. For any Mapping cylinder $W \equiv\left(W_{\check{\phi}}, \pi_{\check{\phi}}, \theta_{\check{\phi}}\right)$, define $\beta_{\check{\phi}} \subset H_{1}\left(W_{\check{\phi}}\right)$ to be the set of homology classes represented by loops which project under $\pi_{\check{\phi}}$ to loops homotopic to

$$
\mathbb{R} / \mathbb{Z} \longrightarrow \mathbb{R} \times \mathbb{R} / \mathbb{Z}, \quad t \longrightarrow(0, t) .
$$

For each $a, b \in[-\infty, \infty]$ where $a<b$, any non-degenerate Hamiltonian $\left(H_{t}\right)_{t \in[0,1]}$ and smooth family of almost complex structures $\left(J_{t}\right)_{t \in[0,1]}$ compatible with $W$ we can define $H F_{[a, b], \beta_{\tilde{\phi}}}^{*}\left(\phi_{1}^{H_{t}}\right)$ in the same way as Floer cohomology of $\phi_{1}^{H_{t}}$ except that we only consider fixed points of action in $[a, b]$ and whose associated 1-periodic orbits represent an element of $\beta_{\check{\phi}}$. We also define $H F_{[-\infty, \infty], \beta_{\tilde{\phi}}^{*}}^{*}\left(\phi_{1}^{H_{t}}\right) \equiv H F_{\beta_{\tilde{\phi}}^{*}}^{*}\left(\phi_{1}^{H_{t}}\right)$.

A non-degenerate autonomous Hamiltonian is an autonomous Hamiltonian $H: W_{\check{\phi}} \longrightarrow \mathbb{R}$ so that for every fixed point $p$ of the time 1 flow $\phi_{1}^{H}$, the eigenspace of $D \phi_{1}^{H}(p)$ associated with the eigenvalue 1 is one dimensional (this eigenspace is the tangent line to the 1-periodic 
orbit associated to $p)$. Now suppose that the mapping cylinder $\left(W_{\check{\phi}}, \pi_{\check{\phi}}, \theta_{\check{\phi}}\right)$ is graded with grading

$$
\iota: \widetilde{F r}\left(T W_{\check{\phi}}\right) \times_{\widetilde{S p}(2 n)} \operatorname{Sp}(2 n) \cong \operatorname{Fr}\left(T W_{\check{\phi}}\right) .
$$

Since any Hamiltonian $H_{t}$ is isotopic to the identity map $\operatorname{id}_{W_{\tilde{\phi}}}$, we get that $\phi_{1}^{H_{t}}$ is naturally graded since the identity map on $\widetilde{F r}\left(T W_{\check{\phi}}\right)$ makes id $_{W_{\check{\phi}}}$ into a graded symplectomorphism. We will call this the standard grading and from now on we will assume that every graded Hamiltonian symplectomorphism has the standard grading. A standard perturbation of a nondegenerate autonomous Hamiltonian $H$ where $\phi_{1}^{H}$ is graded is a time dependent Hamiltonian $\left(H_{t}\right)_{t \in[0,1]}$ which is $C^{\infty}$ close to $H$ and equal to $H$ outside a compact set where

- $\left(H_{t}\right)_{t \in[0,1]}$ is non-degenerate,

- every 1-periodic orbit $\gamma$ of $\left(H_{t}\right)_{t \in[0,1]}$ is a 1-periodic orbit of $H$ and

- for every $S^{1}$ family of 1-periodic orbits $\gamma$ of $H$ there are exactly two 1-periodic orbits $\gamma_{-}$and $\gamma_{+}$in this family which are also 1-periodic orbits of $H_{t}$. These orbits satisfy $C Z\left(\phi_{1}^{H_{t}}, \gamma_{ \pm}\right)=C Z\left(\phi_{1}^{H}, \gamma\right) \pm 1 / 2$.

Such a perturbation exists by [CFH95, Proposition 2.2].

In order to prove our theorem we need another group called $S^{1}$-equivariant Hamiltonian Floer cohomology. See Vit99] and [BO12 for a definition. We will not define this here but we will just state some of the properties that we need. We write these groups as $H F_{S^{1},[a, b], \beta_{\tilde{\phi}}}^{*}(H, J)$ for any non-degenerate autonomous Hamiltonian $H$ and almost complex structures $J$ compatible with $\left(W_{\check{\phi}}, \pi_{\check{\phi}}, \theta_{\check{\phi}}\right)$ and any $a, b \in[-\infty, \infty]$ satisfying $a<b$. We also define $H F_{S^{1}, \beta_{\tilde{\phi}}}^{*}(H, J) \equiv H F_{S^{1},[-\infty, \infty], \beta_{\tilde{\phi}}}^{*}(H, J)$.

These groups satisfy the following properties.

(S1HF1) Let $S_{H}$ be the set of $S^{1}$ families of 1-periodic orbits of $H$ with action in $[a, b]$ representing a class in $\beta_{\check{\phi}}$. Let $\left(H_{t}\right)_{t \in[0,1]}$ be a $C^{\infty}$ small standard perturbation of $H$. This means that for each $\gamma \in S_{H}$, there are two 1-periodic orbits $\gamma_{-}, \gamma_{+}$of $\left(H_{t}\right)_{t \in[0,1]}$ which are also orbits in $\gamma$ satisfying $C Z\left(\phi_{1}^{H_{t}}, \gamma_{ \pm}\right)=C Z\left(\phi_{1}^{H}, \gamma\right) \pm 1 / 2$. Let $S_{H_{t}}$ be the set of such orbits $\gamma_{ \pm}$.

Then the chain complex $C F_{S^{1},[a, b], \beta_{\tilde{\phi}}}^{*}(H)$ defining $H F_{S^{1},[a, b], \beta_{\tilde{\phi}}}^{*}(H, J)$ is a free $\mathbb{Z}[u]$ module generated by $S_{H_{t}}$ and graded by the Conley-Zehnder index taken with negative sign and where the degree of $u$ is -2 . Let $C_{[a, b], \beta_{\tilde{\phi}}}^{*}(H) \subset C_{S^{1},[a, b], \beta_{\tilde{\phi}}}^{*}(H)$ be the $\mathbb{Z}$-submodule generated by elements of $S_{H_{t}}$. Then the differential $\partial$ on $C F_{S^{1},[a, b], \beta_{\dot{\phi}}}^{*}(H)$ is equal to $\partial_{0}+\partial_{1}$ where $\partial_{0}\left(u^{i} C_{[a, b], \beta_{\tilde{\phi}}}^{*}(H)\right) \subset u^{i} C_{[a, b], \beta_{\tilde{\phi}}}^{*}(H)$ and $\partial_{1}\left(u^{i} C_{[a, b], \beta_{\tilde{\phi}}}^{*}(H)\right) \subset$ $\oplus_{j=0}^{i-1} u^{j} C_{[a, b], \beta_{\tilde{\phi}}}^{*}(H)$ for all $i$. Here $\partial_{0}$ is equal to the differential defining $H F_{[a, b], \beta_{\tilde{\phi}}}^{*}(H, J)$. Also $\partial\left(u^{i} \gamma_{-}\right)=u^{i-1} \gamma_{+}$plus 1-periodic orbits of higher action for all $i \geq 1$. The differential is $\mathbb{Z}$-linear but not necessarily $\mathbb{Z}[u]$-linear.

(S1HF2) If $\left(H_{\sigma}, J_{\sigma}\right)_{\sigma \in \mathbb{R}}$ is a smooth family of pairs compatible with $\left(W_{\check{\phi}}, \pi_{\check{\phi}}, \theta_{\check{\phi}}\right)$ with nonincreasing slope joining $(H, J)$ and $(\check{H}, \breve{J})$ then there is a group homomorphism

$$
H F_{S^{1}, \beta_{\check{\phi}}}^{*}(\check{H}, \check{J}) \longrightarrow H F_{S^{1}, \beta_{\check{\phi}}}^{*}(H, J) \text {. }
$$

If in addition $\frac{d}{d \sigma} H_{\sigma} \leq 0$, then we have a group homomorphism

$$
H F_{S^{1},[a, b], \beta_{\check{\phi}}}^{*}(\check{H}, \check{J}) \longrightarrow H F_{S^{1},[a, b], \beta_{\dot{\phi}}}^{*}(H, J)
$$


for all $a<b$. These are called continuation maps. They do not depend on the choice of path $\left(H_{\sigma}, J_{\sigma}\right)_{\sigma \in \mathbb{R}}$ and the composition of two continuation maps is a continuation map. Also if $\left(H_{\sigma}, J_{\sigma}\right)$ does not depend on $\sigma \in \mathbb{R}$ then the associated continuation map is the identity map. If $a=-\infty$ and $b=\infty$ and if $H_{\sigma}=H+f(\sigma)$ for some function $f: \mathbb{R} \longrightarrow \mathbb{R}$ then the corresponding continuation map is also an isomorphism.

(S1HF3) If $(H, J),(\check{H}, \check{J})$ are compatible with $W \equiv\left(W_{\check{\phi}}, \pi_{\check{\phi}}, \theta_{\check{\phi}}\right)$ and

- satisfy the maximum principle with respect to some $V \subset W_{\check{\phi}}$,

- all of $H, \check{H}$ 's 1 -periodic orbits of action in $[a, b]$ representing elements of $\beta_{\check{\phi}}$ are contained in $V$ and

- $\left.(H, J)\right|_{V}=\left.(\check{H}, \check{J})\right|_{V}$,

then

$$
H F_{S^{1},[a, b], \beta_{\check{\phi}}}^{*}(\check{H}, \check{J}) \cong H F_{S^{1},[a, b], \beta_{\check{\phi}}}^{*}(H, J) .
$$

This is due to the fact that their chain complexes are identical. Also if we have two additional pairs $\left(H^{\prime}, J^{\prime}\right),\left(\check{H}^{\prime}, \check{J}^{\prime}\right)$ satisfying the same properties and a smooth nondecreasing family of pairs $\left(H_{\sigma}^{\prime}, J_{\sigma}^{\prime}\right)_{\sigma \in \mathbb{R}}$ and $\left(\check{H}_{\sigma}^{\prime}, \check{J}_{\sigma}^{\prime}\right)_{\sigma \in \mathbb{R}}$ compatible with $W$ joining $(H, J)$ and $\left(H^{\prime}, J^{\prime}\right)$ and joining $(\check{H}, \check{J})$ and $\left(\check{H}^{\prime}, \check{J}\right)^{\prime}$ respectively, satisfying the maximum principle with respect to $V$ and which are equal inside $V$ for all $\sigma$ then the induced continuation maps commute with the above isomorphisms.

Definition 8.10. We define

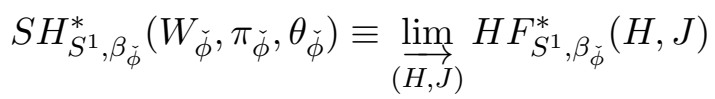

where the direct limit is taken over all pairs $(H, J)$ compatible with $\left(W_{\check{\phi}}, \pi_{\tilde{\phi}}, \theta_{\check{\phi}}\right)$ using the partial ordering $\leq$ on $H$.

Let $\preceq$ be a partial order on a set $S$. A cofinal family is a subset $S^{\prime} \subset S$ so that for all $s \in S$, there exists an $s^{\prime} \in S^{\prime}$ so that $s \preceq s^{\prime}$. In the definition above, it is sufficient to compute $S H_{S^{1}, \beta_{\check{\phi}}}^{*}\left(W_{\check{\phi}}, \pi_{\check{\phi}}, \theta_{\check{\phi}}\right)$ by taking the direct limit over some cofinal family of pairs $(H, J)$ as above.

Lemma 8.11. If the slope of a pair $(H, J)$ compatible with $\left(W_{\check{\phi}}, \pi_{\check{\phi}}, \theta_{\check{\phi}}\right)$ is greater than 1 then the natural map

$$
H F_{S^{1}, \beta_{\check{\phi}}}^{*}(H, J) \longrightarrow S H_{S^{1}, \beta_{\tilde{\phi}}}^{*}\left(W_{\check{\phi}}, \pi_{\check{\phi}}, \theta_{\check{\phi}}\right)
$$

is an isomorphism.

Proof. Let $(\check{H}, \check{J})$ be a pair which is strictly compatible with our mapping cylinder and so that the slope of $\check{H}$ is equal to the slope of $H$. Then there is a constant $c>0$ so that $\check{H}+c>H$ and $H+c>\check{H}$. Consider the continuation maps

$$
H F_{S^{1}, \beta_{\breve{\phi}}}^{*}(H, J) \stackrel{\alpha}{\longrightarrow} H F_{S^{1}, \beta_{\tilde{\phi}}}^{*}(\check{H}+c, \check{J}) \longrightarrow H F_{S^{1}, \beta_{\check{\phi}}}^{*}(H+2 c, J) \longrightarrow H F_{S^{1}, \beta_{\check{\phi}}}^{*}(\check{H}+3 c, \check{J}) \text {. }
$$

By (S1HF2), the composition of any two such maps is an isomorphism and hence the continuation map $\alpha$ is an isomorphism. Therefore it is sufficient for us to assume that $(H, J)$ is strictly compatible with our mapping cylinder. We can also assume that $H=\pi_{\phi}^{*} h(s)$ where $h^{\prime \prime}(s) \geq 0$.

Choose $S>0$ large enough so that $\pi_{\tilde{\phi}}^{-1}((-S, S) \times \mathbb{R} / \mathbb{Z})$ contains all the 1-periodic orbits of $H$ representing a class in $\beta_{\check{\phi}}$. Let $h_{\sigma}: \mathbb{R} \longrightarrow \mathbb{R}, \sigma \in[0, \infty)$ be a smooth family of functions so that 
- $h_{\sigma}(s)=h(s)$ for all $s \in(-S, S)$ and $h_{0}(s)=h(s)$ for all $s \in \mathbb{R}$,

- $h_{\sigma}^{\prime}(s), h_{\sigma}^{\prime \prime}(s), \frac{d}{d \sigma} h_{\sigma}(s) \geq 0$,

- $h_{\sigma}^{\prime \prime}(s)=0$ for all large enough $s$ and

- the slope of $h_{\sigma}$ tends to infinity as $\sigma$ tends to infinity.

By (S1HF3) combined with Lemma 8.7, the natural continuation map

$$
H F_{S^{1}, \beta_{\check{\phi}}}^{*}(H, J) \longrightarrow H F_{S^{1}, \beta_{\tilde{\phi}}}^{*}\left(\pi_{\dot{\phi}}^{*} h_{\sigma}(s), J\right)
$$

is an isomorphism for all $\sigma \geq 0$. Also by (S1HF2), the natural continuation map

$$
H F_{S^{1}, \beta_{\tilde{\phi}}}^{*}(H, J) \longrightarrow H F_{S^{1}, \beta_{\tilde{\phi}}}^{*}\left(\pi_{\tilde{\phi}}^{*} h_{\sigma}(s)+\sigma, J\right)
$$

is an isomorphism for all $\sigma \geq 0$. Since $\left(\pi_{\check{\phi}}^{*} h_{\sigma}(s)+\sigma, J\right)$ is a cofinal family of pairs with respect to the ordering $\leq$, we get our result by (S1HF2).

Lemma 8.12. Fix $q \in \mathbb{R}$. We have

$$
S H_{S^{1}, \beta_{\tilde{\phi}}}^{*}\left(W_{\check{\phi}}, \pi_{\check{\phi}}, \theta_{\check{\phi}}\right)=\underset{(\overrightarrow{H, J})}{\lim _{\longrightarrow}} H F_{S^{1},(-\infty, 0], \beta_{\check{\phi}}}^{*}(H, J)
$$

where the direct limit is taken over pairs $(H, J)$ compatible with our mapping cylinder satisfying $\left.H\right|_{\pi_{\tilde{\phi}}^{-1}((-\infty, q] \times \mathbb{R} / \mathbb{Z})}<0$.

Proof. Since the continuation map

$$
H F_{S^{1}, \beta_{\tilde{\phi}}}^{*}(H, J) \longrightarrow H F_{S^{1}, \beta_{\tilde{\phi}}}^{*}(H+c, J)
$$

is an isomorphism by (S1HF2) for every pair $(H, J)$ compatible with our mapping cylinder, we have

$$
\left.S H_{S^{1}, \beta_{\check{\phi}}}^{*}\left(W_{\check{\phi}}, \pi_{\check{\phi}}, \theta_{\check{\phi}}\right)=\underset{(\overrightarrow{H, J})}{\lim _{\left(S^{1}, \beta_{\check{\phi}}\right.}} H F^{*} H^{\prime} J\right)
$$

where the direct limit is taken over pairs $(H, J)$ compatible with our mapping cylinder satisfying $\left.H\right|_{\pi_{\tilde{\phi}}^{-1}((-\infty, q] \times \mathbb{R} / \mathbb{Z})}<0$. Let $A$ be a constant smaller than the action of all the 1-periodic orbits of $\dot{\phi}$. We say that $h: \mathbb{R} \longrightarrow \mathbb{R}, i \in \mathbb{N}$ is a compatible function with respect to $q$ if

- $h^{\prime}, h^{\prime \prime} \geq 0, h$ is bounded below,

- if $h^{\prime}(x)=1$ then $h(x)<x+\kappa A$ and

- $\left.h_{i}\right|_{(-\infty, q]}<0$.

Then

$$
S H_{S^{1}, \beta_{\check{\phi}}}^{*}\left(W_{\check{\phi}}, \pi_{\check{\phi}}, \theta_{\check{\phi}}\right)=\underset{h}{\lim } H F_{S^{1}, \beta_{\check{\phi}}}^{*}\left(\pi_{\check{\phi}}^{*} h(s), J\right)
$$

where the direct limit is taken over compatible functions with respect to $q$ ordered by $\leq$ and where $J$ is an almost complex structure compatible with our mapping cylinder. Since $\pi_{\phi}^{*} h(s)$ has no 1-periodic orbits representing $\beta_{\breve{\phi}}$ of positive action where $h$ is any compatible function with respect to $q$ we have by (S1HF3).

$$
\underset{h}{\lim _{h}} H F_{S^{1}, \beta_{\tilde{\phi}}}^{*}\left(\pi_{\tilde{\phi}}^{*} h(s), J\right)=\underset{h}{\lim _{h}} H F_{S^{1},(-\infty, 0], \beta_{\tilde{\phi}}}^{*}\left(\pi_{\tilde{\phi}}^{*} h(s), J\right)
$$

proving our result. 
Lemma 8.13. Let $q \in \mathbb{R}$. Let $(\check{H}, \check{J})$ be compatible with $\left(W_{\check{\phi}}, \pi_{\check{\phi}}, \theta_{\check{\phi}}\right)$ then

$$
S H_{S^{1}, \beta_{\check{\phi}}}^{*}\left(W_{\check{\phi}}, \pi_{\check{\phi}}, \theta_{\check{\phi}}\right)=\underset{(\overrightarrow{H, J})}{\lim _{S^{1},(-\infty, 0], \beta_{\check{\phi}}}} H F^{*}(H, J)
$$

where the direct limit is taken over pairs $(H, J)$ compatible with our mapping cylinder satisfying $\left.H\right|_{\pi_{\tilde{\phi}}^{-1}((-\infty, q] \times \mathbb{R} / \mathbb{Z})}<0$ and $\left.(H, J)\right|_{\pi_{\tilde{\phi}}^{-1}([q+1, \infty) \times \mathbb{R} / \mathbb{Z})}=\left.\left(\check{H}+C_{H}, J\right)\right|_{\pi_{\tilde{\phi}}^{-1}([q+1, \infty) \times \mathbb{R} / \mathbb{Z})}$ for some constant $C_{H} \in \mathbb{R}$.

Proof. Let $\left(H_{i}, J_{i}\right)_{i \in \mathbb{N}}$ be a cofinal family of pairs with respect to the directed system mentioned in the statement of this lemma. Such a countable family exists since

$$
\left.\sup H\right|_{\pi_{\tilde{\phi}}^{-1}((-\infty, q] \times(\mathbb{R} / \mathbb{Z}))}<0
$$

for any $(H, J)$ in the directed system above. We have that $\left(H_{i}, J_{i}\right)$ is compatible with our mapping cylinder and $\left.H_{i}\right|_{\left(\pi_{\tilde{\phi}}^{-1}((-\infty, q] \times \mathbb{R} / \mathbb{Z})\right)}<0$ and $\left.\left(H_{i}, J_{i}\right)\right|_{\pi_{\tilde{\phi}}^{-1}([q+1, \infty) \times \mathbb{R} / \mathbb{Z})}=(\check{H}+$ $\left.C_{H_{i}}, J_{i}\right)\left.\right|_{\left.\pi_{\tilde{\phi}}^{-1}([q+1, \infty) \times \mathbb{R} / \mathbb{Z})\right)}$ for some constant $C_{H_{i}} \in \mathbb{R}$. We can assume that $H_{i}<H_{i+1}$ and hence $C_{H_{i}}<C_{H_{i+1}}$ for all $i \in \mathbb{N}$. After passing to a subsequence, we can assume that $C_{H_{i}}>i$ for all $i \in \mathbb{N}$.

Let $(s, t)$ be standard coordinates for the base $\mathbb{R} \times \mathbb{R} / \mathbb{Z}$. Let $K: W_{\check{\phi}} \longrightarrow \mathbb{R}$ be a Hamiltonian equal to $\pi_{\tilde{\phi}}^{*} k(s)$ where $k(s)=0$ for $s \leq q+1, k^{\prime}(s)>0$ for $s>q+1$, and $k^{\prime}(s)$ is constant for $s>q+2$. Since $C_{H_{i}}>i$ for all $i$, there is a $\delta>0$ small enough so that the set of 1-periodic orbits of $H_{i}$ of non-positive action are equal to the set of 1-periodic orbits of $H_{i}+\delta i K$ of non-positive action. Hence by (S1HF3)

$$
H F_{S^{1},(-\infty, 0], \beta_{\tilde{\phi}}}^{*}\left(H_{i}, J_{i}\right)=H F_{S^{1},(-\infty, 0], \beta_{\tilde{\phi}}}^{*}\left(H_{i}+\delta i K, J_{i}\right) .
$$

Combining this with Lemma 8.13 gives us our result.

Lemma 8.14. Suppose that the mapping cylinders $\left(W_{\check{\phi}_{1}}, \pi_{\check{\phi}_{1}}, \theta_{\check{\phi}_{1}}\right),\left(W_{\check{\phi}_{2}}, \pi_{\check{\phi}_{2}}, \theta_{\check{\phi}_{2}}\right)$ are isomorphic. Then

$$
S H_{S^{1}, \beta_{\check{\phi}_{1}}}^{*}\left(W_{\check{\phi}_{1}}, \pi_{\check{\phi}_{1}}, \theta_{\check{\phi}_{1}}\right)=S H_{S^{1}, \beta_{\check{\phi}_{2}}}^{*}\left(W_{\check{\phi}_{2}}, \pi_{\check{\phi}_{2}}, \theta_{\check{\phi}_{2}}\right) .
$$

Proof. Since these mapping cylinders are isomorphic, we can assume that $W_{\check{\phi}_{1}}=W_{\check{\phi}_{2}}, \pi_{\check{\phi}_{2}}=$ $\pi_{\check{\phi}_{2}}$ inside $\left\{r_{\check{\phi}_{1}} \geq 1-\delta\right\}$ for some $\delta>0$ and $\theta_{\check{\phi}_{1}}=\theta_{\check{\phi}_{2}}+k$ where $k: W_{\check{\phi}_{1}} \longrightarrow \mathbb{R}$ has support disjoint from $\left\{r_{\check{\phi}_{1}} \geq 1-\delta\right\}$.

Let $\left(H_{1}, J_{1}\right)$ (resp. $\left.\left(H_{2}, J_{2}\right)\right)$ be a pair strictly compatible with

$$
W_{1} \equiv\left(W_{\check{\phi}_{1}}, \pi_{\check{\phi}_{1}}, \theta_{\check{\phi}_{1}}\right) \quad\left(\text { resp. } W_{2} \equiv\left(W_{\check{\phi}_{2}}, \pi_{\check{\phi}_{2}}, \theta_{\check{\phi}_{2}}\right)\right)
$$

of slope less than some small $\check{\delta}>0$. If $\check{\delta}>0$ is small enough then we can construct a pair $\left(H_{3}, J_{3}\right)$ compatible with $W_{1}$, which is equal to $\left(H_{2}, J_{2}\right)$ in the region $\pi_{\tilde{\phi}_{2}}^{-1}((-1,3) \times \mathbb{R} / \mathbb{Z})$ and equal to $\left(H_{1}, J_{1}\right)$ inside $\pi_{\check{\phi}_{2}}^{-1}(((-\infty,-2),(4, \infty)) \times \mathbb{R} / \mathbb{Z})$ so that $H_{3}$ has no 1 -periodic orbits. Let $\left(\check{H}_{i}, \check{J}_{i}\right)_{i \in \mathbb{N}}$ be a family of pairs strictly compatible with $W_{2}$ so that

- $\left(\check{H}_{i}, \breve{J}_{i}\right)$ is equal to $\left(H_{2}+C_{\check{H}_{i}}, J_{2}\right)$ inside $\pi_{\check{\phi}_{2}}^{-1}([1, \infty) \times \mathbb{R} / \mathbb{Z})$ for some constants $C_{\breve{H}_{i}} \in \mathbb{R}$,

- the restriction $\left.\check{H}_{i}\right|_{\pi_{\tilde{\phi}_{2}}^{-1}((-\infty, 0) \times \mathbb{R} / \mathbb{Z})}$ is negative and uniformly tends to 0 in the $C^{1}$ norm as $i$ tends to infinity and $\check{H}_{i}(x) \rightarrow \infty$ as $i \rightarrow \infty$ for all $x$ in $\pi_{\check{\phi}_{2}}^{-1}((0, \infty) \times \mathbb{R} / \mathbb{Z})$.

Let $\left(\widehat{H}_{i}, \widehat{J}_{i}\right)_{i \in \mathbb{N}}$ be a family of pairs compatible with $W_{1}$ so that

- $\left(\widehat{H}_{i}, \widehat{J}_{i}\right)$ is equal to $\left(H_{3}+C_{\breve{H}_{i}}, \check{J}_{2}\right)$ inside $\pi_{\check{\phi_{1}}}^{-1}([1, \infty) \times \mathbb{R} / \mathbb{Z})$, 
- $\left(\widehat{H}_{i}, \widehat{J}_{i}\right)$ equals $\left(\check{H}_{i}, \check{J}_{i}\right)$ inside $\pi_{\check{\phi}_{2}}^{-1}((-1,3) \times \mathbb{R} / \mathbb{Z})$,

- the restriction $\left.\widehat{H}_{i}\right|_{\pi_{\tilde{\phi}_{1}}^{-1}((-\infty, 0) \times \mathbb{R} / \mathbb{Z})}$ is negative and uniformly tends to 0 in the $C^{1}$ norm as $i$ tends to infinity and $\widehat{H}_{i}(x) \rightarrow \infty$ as $i \rightarrow \infty$ for all $x$ in $\pi_{\check{\phi_{1}}}^{-1}((0, \infty) \times \mathbb{R} / \mathbb{Z})$.

Then by Lemma 8.13 ,

$$
S H_{S^{1}, \beta_{\check{\phi}_{1}}}^{*}\left(W_{\check{\phi}_{2}}, \pi_{\check{\phi}_{2}}, \theta_{\check{\phi}_{2}}\right) \equiv \lim _{i \in \mathbb{N}} H F_{S^{1},(-\infty, 0]}^{*}\left(\check{H}_{i}, \check{J}_{i}\right) .
$$

and

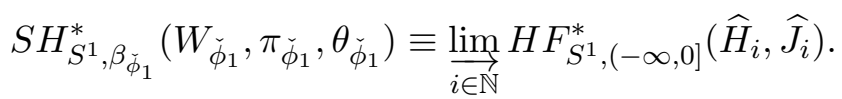

Also by property $(\mathrm{S} 1 \mathrm{HF} 3), H F_{S^{1},(-\infty, 0], \beta_{\check{\phi}_{1}}}^{*}\left(\widehat{H}_{i}, \widehat{J}_{i}\right) \cong H F_{S^{1},(-\infty, 0], \beta_{\check{\phi}_{1}}}^{*}\left(\check{H}_{i}, \check{J}_{i}\right)$ for all $i$ and the continuation maps between these groups commute with these isomorphisms. Hence

$$
S H_{S^{1}, \beta_{\check{\phi}_{1}}}^{*}\left(W_{\check{\phi}_{1}}, \pi_{\check{\phi}_{1}}, \theta_{\check{\phi}_{1}}\right) \cong S H_{S^{1}, \beta_{\check{\phi}_{1}}}^{*}\left(W_{\check{\phi}_{2}}, \pi_{\check{\phi}_{2}}, \theta_{\check{\phi}_{2}}\right)
$$

by Equations (8.4) and (8.3).

Lemma 8.15. Let $A_{-}, A_{+}$be free abelian groups. Define $B_{-} \equiv A_{-} \otimes \mathbb{Z}[u], B_{+} \equiv A_{+} \otimes \mathbb{Z}[u]$. Let

$$
\partial \equiv \partial_{0}+\partial_{1}: B_{-} \oplus B_{+} \longrightarrow B_{-} \oplus B_{+}
$$

be a $\mathbb{Z}$-linear differential where $\partial_{0}\left(A_{-}\right) \subset A_{-}$and $\partial_{1}\left(A_{-}\right)=0$. Now suppose that we have a filtration $B_{-} \oplus B_{+}=F_{0} \supset F_{1} \supset F_{2} \cdots$ for the chain complex $\left(B_{-} \oplus B_{+}, \partial\right)$ so that if $V_{ \pm}^{i} \equiv\left(B_{ \pm} \cap F_{i}\right) /\left(B_{ \pm} \cap F_{i+1}\right)$ then $\partial_{1}\left(u V_{-}^{i}\right) \subset V_{+}^{i}$ and

$$
\left.\partial_{1}\right|_{u V_{-}^{i}}: u V_{-}^{i} \longrightarrow V_{+}^{i}
$$

is an isomorphism for all $i \geq 0$. Then

$$
H\left(B_{-} \oplus B_{+}, \partial\right)=H\left(A_{-}, \partial_{0}\right) .
$$

If these groups are graded then the above isomorphism respects this grading.

Proof. Define $\check{B} \equiv \partial\left(u B_{-}\right)$and define $\partial_{\check{B}}: u B_{-} \longrightarrow \check{B}, \quad \partial_{\check{B}}(x)=\partial(x)$. Since the map (8.5) is an isomorphism for all $i \geq 0$, we get that $\left.\partial_{1}\right|_{u B_{-}}: u B_{-} \longrightarrow\left(B_{-} \oplus B_{+}\right) /\left(B_{-} \oplus 0\right)$ is an isomorphism. Hence $B_{-} \oplus B_{+} \cong A_{-} \oplus u B_{-} \oplus \check{B}$ and the differential with respect to this splitting is the matrix

$$
\left(\begin{array}{ccc}
\partial_{0} & 0 & 0 \\
0 & 0 & 0 \\
0 & \partial_{\check{B}} & 0
\end{array}\right) .
$$

Computing the homology of this chain complex using the above matrix gives us our result since $\partial_{B^{\prime}}$ is an isomorphism.

Lemma 8.16. Let $\left(W_{\check{\phi}}, \pi_{\check{\phi}}, \theta_{\check{\phi}}\right)$ be a mapping cylinder. Then

$$
S H_{\beta_{\check{\phi}}}^{*}\left(W_{\check{\phi}}, \pi_{\check{\phi}}, \theta_{\check{\phi}}\right)=H F^{*}(\phi,+) .
$$

Proof. Let $\left(H=\pi_{\check{\phi}}^{*} h(s), J\right)$ be a pair strictly compatible with $\left(W_{\check{\phi}}, \pi_{\check{\phi}}, \theta_{\check{\phi}}\right)$ where $H$ has slope $1.5, h^{\prime \prime} \geq 0$ and where $\left.h^{\prime}\right|_{(-\infty, 0)}<1$. Let $h_{t}: \mathbb{R} \times \mathbb{R} / \mathbb{Z} \longrightarrow \mathbb{R}, \quad t \in[0,1]$ be a standard perturbation of $h(s)$ viewed as a Hamiltonian on the symplectic manifold $(\mathbb{R} \times \mathbb{R} / \mathbb{Z}, d s \wedge d t$ ). Then $h_{t}$ has exactly two 1-periodic orbits $\gamma_{-}, \gamma_{+}$. Also $H_{t} \equiv \pi_{\bar{\phi}}^{*} h_{t}$ is a standard perturbation 
of $H$ and the 1-periodic orbits of $H_{t}$ project to $\gamma_{-}$or $\gamma_{+}$. A compactness argument $\left(\left[\overline{\mathrm{BEH}^{+} 03}\right)\right.$ tells us that $\left(H_{t}, J\right)$ satisfies the maximum principle so long as $H_{t}$ is sufficiently $C^{\infty}$ close to $H$ and hence we can define $H F_{\beta_{\tilde{\phi}}}^{*}\left(\phi_{1}^{H_{t}}\right)$ in the usual way. We can also define $H F_{S^{1}, \beta_{\tilde{\phi}}}^{*}(H, J)$ using the standard perturbation $H_{t}$ by the same compactness argument.

By [McL12b, Theorem 1.3], we have that $H F_{\beta_{\tilde{\phi}}}^{*}\left(\phi_{1}^{H_{t}}, J\right)$ is isomorphic to $H F_{\beta_{\tilde{\phi}}}^{*}\left(\phi_{1}^{H_{t}},+\right) \oplus$ $H F_{\beta_{\tilde{\phi}}}^{*+1}\left(\phi_{1}^{H_{t}},+\right)$. In fact, in the proof of the above Theorem it was shown that if $A_{-}$(resp. $A_{+}$) is the free abelian group generated by 1-periodic orbits of $H_{t}$ which project to $\gamma_{-}$(resp. $\left.\gamma_{+}\right)$then the differential

$$
\partial_{H_{t}, J}: A_{-} \oplus A_{+} \longrightarrow A_{-} \oplus A_{+}
$$

satisfies $\partial_{H_{t}, J}\left(A_{ \pm}\right) \subset A_{ \pm}$and the homology of

$$
\left.\partial_{H_{t}, J}\right|_{A_{-}}: A_{-} \longrightarrow A_{-}
$$

is equal to $H F^{*}\left(\phi_{1}^{H_{t}}, J\right)$.

If $\check{\phi}$ is a sufficiently generic positive slope perturbation of $\phi$, then we can find a sequence $\left(\alpha_{i}\right)_{i \in \mathbb{N}_{>0}}$ so that there are exactly two 1-periodic orbits $\check{\gamma}_{-}, \check{\gamma}_{+}$of $H_{t}$ of action in the interval $\left[\alpha_{i}, \alpha_{i+1}\right)$ and all 1-periodic orbits are contained in one such interval. Let $B_{ \pm} \equiv A_{ \pm} \otimes \mathbb{Z}[u]$ where $u$ has degree -2 . Let $B_{-} \oplus B_{+}=F_{0} \supset F_{1} \supset \cdots$ be a filtration where $F_{i}$ is the $\mathbb{Z}[u]$ submodule generated by orbits of action $\geq \alpha_{i}$. Define $V_{ \pm}^{i} \equiv\left(B_{ \pm} \cap F_{i}\right) /\left(B_{ \pm} \cap F_{i+1}\right)$ for all $i \in \mathbb{N}_{\geq 0}$. By [S1HF1), the differential $\partial: B_{-} \oplus B_{+} \longrightarrow B_{-} \oplus B_{+}$computing $H F_{S^{1}, \beta_{\tilde{\phi}}}^{*}(H, J)$ is equal to $\partial_{0}+\partial_{1}$ where $\partial_{0}\left(A_{-}\right) \subset A_{-}, \partial_{1}\left(A_{-}\right)=0,\left.\partial_{0}\right|_{A_{-}}=\partial_{H_{t}, J}, \partial_{1}\left(u V_{-}^{i}\right) \subset V_{+}^{i}$ and $\left.\partial_{1}\right|_{u V_{-}^{i}}: u V_{-}^{i} \longrightarrow V_{+}^{i}$ is an isomorphism for all $i \in \mathbb{N}_{\geq 0}$.

Therefore by Lemma 8.15 we have that $H F_{S^{1}, \beta_{\tilde{\phi}}}^{*}(H, J)=H\left(A_{-}, \partial_{0}\right)=H F^{*}(\phi,+)$. Also $H F_{S^{1}, \beta_{\check{\phi}}}^{*}(H, J)=S H_{S^{1}, \beta_{\check{\phi}}}^{*}\left(W_{\check{\phi}}, \pi_{\check{\phi}}, \theta_{\check{\phi}}\right)$ by Lemma 8.11 and hence $S H_{S^{1}, \beta_{\check{\phi}}}^{*}\left(W_{\check{\phi}}, \pi_{\check{\phi}}, \theta_{\check{\phi}}\right)=$ $H F^{*}(\phi,+)$.

Proof of Property (HF2), This follows from Lemmas 8.2, 8.3, 8.14 and 8.16,

Now the only issue is that if we have two polynomials with embedded contactomorphic links. Then we need to show that the associated contact pairs are isomorphic. In other words, we need to show that the normal bundles coincide up to homotopy. This is contained in the proof of the following lemma.

Lemma 8.17. Let $f, g: \mathbb{C}^{n+1} \longrightarrow \mathbb{C}$ be a polynomials with isolated singularities at 0 with embedded contactomorphic links where $n \geq 1$. Then $H F^{*}\left(\phi^{m},+\right)=H F^{*}\left(\psi^{m},+\right)$ where $\phi$ (resp. $\psi$ ) is the monodromy map of the Milnor open book associated to $f$ (resp. $g$ ) as in Example 3.11 .

Proof. Let $\left(L_{f} \subset S_{\epsilon}, \xi_{S_{\epsilon}}, \Phi_{f}\right)$ and $\left(L_{g} \subset S_{\epsilon}, \xi_{S_{\epsilon}}, \Phi_{g}\right)$ be the contact pairs associated to $f$ and $g$ respectively as in Example 3.8. Let $\Psi: S_{\epsilon} \longrightarrow S_{\epsilon}$ be the contactomorphism sending $L_{f}$ to $L_{g}$. We need to show that $\Psi$ is in fact a contactomorphism of graded contact pairs by $\left(\right.$ HF2). Since $H_{1}\left(S_{\epsilon} ; \mathbb{Q}\right)=0$, we get that $\Psi$ is a graded contactomorphism by Equation (7.2) in Definition [7.7. Therefore we just need to show that the composition

$$
\mathcal{N}_{S_{\epsilon}} L_{f} \stackrel{\left.d \Psi\right|_{L_{f}}}{\longrightarrow} \mathcal{N}_{S_{\epsilon}} L_{g} \stackrel{\Phi_{g}}{\longrightarrow} L_{g} \times \mathbb{C} \stackrel{\Psi^{-1} \times \mathrm{id}_{\mathbb{C}}}{\longrightarrow} L_{f} \times \mathbb{C}
$$

is homotopic to $\Phi_{f}$.

This is true since the trivialization $\Phi_{f}$ (and similarly $\Phi_{g}$ ) is uniquely determined by the following topological property: Let $\Psi_{f}: \check{\mathcal{N}}_{S_{\epsilon}} L_{f} \longrightarrow S_{\epsilon}$ be a regularization of $L_{f}$ as in 
Definition 5.2. Then the trivialization $\Phi_{f}$ gives us a section $s$ of $\check{\mathcal{N}}_{S_{\epsilon}} L_{f}$ whose image under the trivialization $\Phi_{f}$ is a constant section. Then $s$ is the unique section up to homotopy with the property that the image of $H_{1}\left(L_{f} ; \mathbb{Q}\right) \stackrel{\Psi_{f} \circ s}{\longrightarrow} H_{1}\left(S_{\epsilon}-L_{f} ; \mathbb{Q}\right)$ is zero. This trivialization could be thought of as a generalization of the Seifert framing of links.

\section{Appendix C: A Morse-Bott Spectral Sequence}

In this section, we will show that property (HF3) holds. Here is a statement of this property:

Let $\left(M, \theta_{M}, \phi\right)$ be a graded abstract contact open book where $\operatorname{dim}(M)=2 n$. Suppose that the set of fixed points of a small positive slope perturbation $\dot{\phi}$ of $\phi$ is a disjoint union of codimension 0 families of fixed points $B_{1}, \cdots, B_{l}$ and let $\iota:\{1, \cdots, l\} \rightarrow \mathbb{N}$ be a function where

- $\iota(i)=\iota(j)$ if and only if the action of $B_{i}$ equals the action of $B_{j}$ and

- $\iota(i)<\iota(j)$ if the action of $B_{i}$ is less than the action of $B_{j}$.

Then there is a cohomological spectral sequence converging to $H F^{*}(\phi,+)$ whose $E_{1}$ page is equal to

$$
E_{1}^{p, q}=\bigoplus_{\{i \in\{1, \cdots, l\}: \iota(i)=p\}} H_{n-(p+q)-C Z\left(\phi, B_{j}\right)}\left(B_{p} ; \mathbb{Z}\right) .
$$

The spectral sequence above is an example of a Morse-Bott spectral sequence. Before we prove this statement we need some preliminary definitions and lemmas.

Definition 9.1. $\left(M, \theta_{M}, \phi\right)$ be a graded abstract contact open book. Let $\check{\phi}: M \longrightarrow M$ be a small positive slope perturbation of $\phi$ and $\left(J_{t}\right)_{t \in[0,1]}$ a $C^{\infty}$ generic family of $d \theta_{M}$-compatible almost complex structures. Let $a, b \in \mathbb{R}$ be real numbers with the property that no fixed point of $\check{\phi}$ has action equal to $a$ or $b$.

We define $H F_{[a, b]}^{*}\left(\check{\phi},\left(J_{t}\right)_{t \in[0,1]}\right)$ in the following way: Let $\check{\phi}^{\prime}$ be a $C^{\infty}$ small generic perturbation of $\check{\phi}$ inside a compact set so that all of the fixed points of $\check{\phi}^{\prime}$ are non-degenerate. Then $H F_{([a, b]}^{*}\left(\check{\phi},\left(J_{t}\right)_{t \in[0,1]}\right)$ is defined in the same way as $H F^{*}\left(\check{\phi}^{\prime},\left(J_{t}\right)_{t \in[0,1]}\right)$ except that we only consider orbits inside the action window $[a, b]$. This group does not depend on the choice of perturbation $\check{\phi}^{\prime}$ so long as no fixed point of $\check{\phi}$ has action equal to $a$ or $b$.

We can define this group in the following equivalent way: Let $C F^{*}\left(\check{\phi}^{\prime}\right)$ be the chain complex for $\check{\phi}^{\prime}$. Then the subspace $C F_{[a, \infty]}^{*}\left(\check{\phi}^{\prime}\right)$ consisting of fixed points of action $\geq a$ is a subcomplex. We define $H F_{[a, b]}^{*}\left(\check{\phi}^{\prime},\left(J_{t}\right)_{t \in[0,1]}\right)$ to be the homology of the quotient complex $C F_{[a, \infty]}^{*}\left(\check{\phi}^{\prime}\right) / C F_{[b, \infty]}^{*}\left(\check{\phi}^{\prime}\right)$.

Suppose that $B$ is the set of fixed points of $\check{\phi}$ of action $c$ and suppose that there is some $a<c<b$ so that there are no fixed points of action in $[a, b]-c$. We define

$$
H F^{*}(\check{\phi}, B) \equiv H F_{[a, b]}^{*}\left(\check{\phi},\left(J_{t}\right)_{t \in[0,1]}\right) .
$$

This does not depend on the choice of $a, b$ or $\left(J_{t}\right)_{t \in[0,1]}$.

Lemma 9.2. $\left(M, \theta_{M}, \phi\right)$ be a graded abstract contact open book. Let $\check{\phi}: M \longrightarrow M$ be the composition of $\phi$ with a $C^{\infty}$ small Hamiltonian so that $\check{\phi}$ has small positive slope. Let $B \subset M$ be an isolated family of fixed points of $\check{\phi}$. 
Let $\left(J_{t}\right)_{t \in[0,1]}$ be a smooth family of almost complex structures cylindrical near $\partial M$. Then there is an neighborhood $N_{B} \subset M$ of $B$ so that for any sufficiently small $C^{\infty}$ perturbation $\check{\phi}^{\prime}$, any Floer trajectory of $\left(\check{\phi}^{\prime},\left(J_{t}\right)_{t \in[0,1]}\right)$ connecting non-degenerate fixed points $p, \check{p} \in N_{B}$ of $\check{\phi}^{\prime}$ is contained inside $N_{B}$.

Proof. We choose a relatively compact open neighborhood $N_{B}$ of $B$ so that any fixed point of $\check{\phi}$ inside $\overline{N_{B}}$ is actually contained inside $B$. Let $\check{N}_{B} \subset M$ be an open neighborhood of $B$ whose closure is contained in $N_{B}$.

Let $\left(\phi_{k}\right)_{k \in \mathbb{N}}$ be a sequence of symplectomorphisms of $M$ which $C^{\infty}$ converge to $\check{\phi}$. Suppose (for a contradiction) that $\phi_{k}$ has a fixed point $p_{k} \in N_{B}-\check{N}_{B}$ for all $k$. Then after passing to a subsequence, we have that $p_{i}$ converges to some $p \in \overline{N_{B}}-\check{N}_{B}$. Since $p$ is a fixed point of $\phi$, we get that $p \in B$ which is impossible. Therefore $\phi_{k}$ has no fixed points inside $N_{B}-\check{N}_{B}$ for all sufficiently large $k$.

Now suppose that $p_{k}, \check{p}_{k}$ are fixed points of $\phi_{k}$ and suppose that we have a sequence of Floer trajectories

$$
u_{k}: \mathbb{R} \times[0,1] \longrightarrow M
$$

of $\left(\check{\phi}^{\prime},\left(J_{t}\right)_{t \in[0,1]}\right)$ joining $p_{k}$ and $\check{p}_{k}$. Define $W \equiv \mathbb{R} \times[0,1] \times M$ with a symplectic form $\omega_{W} \equiv d s \wedge d t+d \theta_{M}$ where $s, t$ are the standard coordinates on $\mathbb{R} \times[0,1]$. Let $i$ be the standard complex structure on $\mathbb{R} \times[0,1] \subset \mathbb{C}$ where $(s, t)$ is identified with $s+i t$. Define $\left.\left.\left.J^{W}\right|_{(s, t, x)} \equiv i\right|_{(s, t)} \oplus J_{t}\right|_{(s, t)}$. Define

$$
u_{k}^{W}: \mathbb{R} \times[0,1] \longrightarrow W, \quad u_{k}^{W}(s, t) \equiv\left(s, t, u_{k}(s, t)\right)
$$

for all $k \in \mathbb{N}$. This is a sequence of $J^{W}$-holomorphic maps.

Now suppose (for a contradiction) that the image of $u_{k}$ is not contained inside $N_{B}$ for all $k$. Then after passing ot a subsequence, there is a sequence of points $\left(s_{k}, t_{k}\right) \in \mathbb{R} \times[0,1]$ so that $u_{k}\left(s_{k}, t_{k}\right) \in N_{B}-\check{N}_{B}$ and $u_{k}\left(s_{k}, t_{k}\right)$ converges to some point $q \in \bar{N}_{B}-\check{N}_{B}$. After reparameterizing the domain by translations in the $s$ direction, we can assume that $s_{k}=0$ for all $k$. Also after passing to a subsequence we can assume that $t_{k} \longrightarrow \check{t} \in[0,1]$ for some $\check{t}$. Define $\left.w_{k} \equiv u_{k}^{W}\right|_{[-1,1] \times[0,1]}$. Then by the main result in [Fis11], we get that $w_{k} C^{0}$ converges to a continuous map $v:[-1,1] \times[0,1] \longrightarrow W$ which is smooth and $J^{W}$-holomorphic on a dense open subset of its domain.

Let $\pi_{M}: W \longrightarrow M$ be the natural projection map. Since $p_{k}$ and $\check{p}_{k}$ converge to points in $B$, their difference in action converges to zero which implies that $\int_{[-1,1] \times \mathbb{R} / \mathbb{Z}} v^{*} d \theta_{M}=0$. Hence $\pi_{M} \circ v$ is constant. Since $\check{\phi}\left(\pi_{M}(v(0,1))\right)=\pi_{M}(v((0,0)))$ and $\pi_{M} \circ v$ is constant, we get that the image of $\pi_{M} \circ v$ is a fixed point of $\check{\phi}$ inside $N_{B}-\check{N}_{B}$. But this is impossible since $v(0, t) \in N_{B}-\check{N}_{B}$.

As a result of the above lemma, we have the following definition:

Definition 9.3. Let $B \subset M$ be an isolated family of fixed points of some positive slope perturbation $\check{\phi}$ of $\phi$ and let $N_{B}$ be a neighborhood of $B$ as in Lemma 9.2, Let $\check{\phi}^{\prime}$ be a $C^{\infty}$ small perturbation so that all the fixed points of $\check{\phi}^{\prime}$ inside $N_{B}$ are non-degenerate. Since all Floer trajectories of $\left(\check{\phi}^{\prime},\left(J_{t}\right)_{t \in[0,1]}\right)$ are contained inside $N_{B}$, we can define the Floer cohomology group $H F^{*}(\check{\phi}, B)$ to be defined in the usual way where we only consider fixed points inside $N_{B}$. Such a group is called the local Floer cohomology of B. Again it does not depend on the choice of perturbation $\check{\phi}^{\prime}$ or $\left(J_{t}\right)_{t \in[0,1]}$ although we will not need this fact here.

Note that if $B$ is the only set of fixed points of $\check{\phi}$ of action in the interval $[a, b]$, then the above definition coincides with the definition of $H F^{*}(\check{\phi}, B)$ from Definition 9.1. More 
generally, if $B$ is a union of isolated families of fixed points $B_{1}, \cdots, B_{l}$ all of the same action then $H F^{*}(\check{\phi}, B)=\bigoplus_{i=1}^{l} H F^{*}\left(\check{\phi}, B_{i}\right)$.

Lemma 9.4. $\left(M, \theta_{M}, \phi\right)$ be a graded abstract contact open book. Let $\check{\phi}: M \longrightarrow M$ be a small positive slope perturbation of $\phi$. Suppose that all the fixed points of $\check{\phi}$ of action in $[a, b]$ is equal to $B=\sqcup_{i=1}^{l} B_{i}$ where $B_{1}, \cdots, B_{l}$ are codimension 0 families of fixed points, all of the same action. Then

$$
H F^{*}(\check{\phi}, B) \equiv \bigoplus_{i=1}^{l} H_{n-*-C Z\left(\phi, B_{i}\right)}\left(B_{i} ; \mathbb{Z}\right) .
$$

Proof. Let $N_{B_{i}} \subset M$ be a small neighborhood of $B_{i}$ with the property that $\check{\phi}$ is the time 1 flow of a Hamiltonian $H_{B_{i}}: N_{B_{i}} \longrightarrow(-\infty, 0]$ satisfying $B_{i}=H_{B_{i}}^{-1}(0)$ for each $i$. Let $\phi_{1}^{H_{B_{i}}}$ : $N_{B_{i}} \longrightarrow N_{B_{i}}$ be the time 1 flow of $H_{B}$ for each $i$. After possibly shrinking each neighborhood $N_{B_{i}}$, we can assume that $N_{B_{1}}, \cdots, N_{B_{l}}$ are all disjoint. Let $\left(J_{t}\right)_{t \in[0,1]}$ be a generic smooth family almost complex structures cylindrical near $\partial M$. By Lemma 9.2, any sufficiently small $C^{\infty}$ perturbation $\check{\phi}^{\prime}$ of $\check{\phi}$ has the property that any Floer trajectory connecting fixed points inside $\cup_{i=1}^{l} N_{B_{i}}$ is actually contained inside $N_{B_{j}}$ for some $j$. Therefore

$$
H F^{*}(B)=\bigoplus_{i=1}^{l} H F^{*}\left(\phi_{1}^{H_{B_{i}}}, B_{i}\right) .
$$

Hence by [SZ92, Theorem 7.1] combined with (CZ4), we have that Equation (9.2) holds.

Proof of Property (HF3), Let $\check{\phi}^{\prime}$ be a $C^{\infty}$ small perturbation of $\check{\phi}$ and let $\left(J_{t}\right)_{t \in[0,1]}$ be a $C^{\infty}$ generic smooth family of almost complex structures cylindrical near $\partial M$. Let $\alpha_{i}$ be the action of $B_{i}$ for each $i \in\{1, \cdots, l\}$. For each $p \in \mathbb{N}$, choose $\beta_{p} \in \mathbb{R}$ so that $\alpha_{i} \neq \beta_{p}$ for all $i \in\{1, \cdots, l\}$ and so that $\alpha_{i}>\beta_{p}$ if and only if $\iota(i) \geq p$. Let $F_{p}$ be the subgroup of the chain complex $C F^{*}\left(\check{\phi}^{\prime}\right)$ generated by fixed points of action greater than $\beta_{p}$. Then $\left(F_{p}\right)_{i \in p}$ is a filtration on this chain complex. By Lemma 9.4,

$$
H^{*}\left(F_{p} / F_{p-1}\right)=H F_{\left[\beta_{p-1}, \beta_{p}\right]}^{*}\left(\check{\phi}, B_{p}\right)=\bigoplus_{\{i \in\{1, \cdots, l\}: \iota(i)=p\}} H_{n-*-C Z\left(\phi, B_{j}\right)}\left(B_{p} ; \mathbb{Z}\right)
$$

for all $p=1, \cdots, l$ and $H^{*}\left(F_{p} / F_{p-1}\right)=0$ if $p \in \mathbb{N}-\{1, \cdots, l\}$. Therefore the spectral sequence associated to the filtration $\left(F_{p}\right)_{p \in \mathbb{N}}$ is (9.1).

\section{REFERENCES}

[A'C75] Norbert A'Campo. La fonction zeta d'une monodromie. Comment. Math. Helv., 50:233-248, 1975.

[AS10] Mohammed Abouzaid and Paul Seidel. An open string analogue of Viterbo functoriality. Geom. Topol., 14(2):627-718, 2010.

[Ati70] Michael Atiyah. Resolution of singularities and division of distributions. Comm. Pure Appl. Math., 23:145-150, 1970.

$\left[\mathrm{BEH}^{+} 03\right]$ Frederic Bourgeois, Yakov Eliashberg, Helmut Hofer, Krzysztof Wysocki, and Eduard Zehnder. Compactness results in symplectic field theory. Geom. Topol., 7:799-888, 2003.

[BO12] Frédéric Bourgeois and Alexandru Oancea. $S^{1}$-equivariant symplectic homology and linearized contact homology. pages 1-67, 2012, arXiv:1212.3731.

[Bot57] Raoul Bott. The stable homotopy of the classical groups. Proc. Nat. Acad. Sci. U.S.A., 43:933-935, 1957.

[Bud12] Nero Budur. Singularity invariants related to Milnor fibers: survey. In Zeta functions in algebra and geometry, volume 566 of Contemp. Math., pages 161-187. Amer. Math. Soc., Providence, RI, 2012. 
[BW58] William Boothby and Hsieu-chung Wang. On contact manifolds. Ann. of Math. (2), 68:721-734, 1958.

[CFH95] Kai Cieliebak, Andreas Floer, and Helmut Hofer. Symplectic homology. II. A general construction. Math. Z., 218(1):103-122, 1995.

[CNPP06] Clément Caubel, András Némethi, and Patrick Popescu-Pampu. Milnor open books and Milnor fillable contact 3-manifolds. Topology, 45(3):673-689, 2006.

[CZ84] Charles Conley and Eduard Zehnder. Morse-type index theory for flows and periodic solutions for Hamiltonian equations. Comm. Pure Appl. Math., 37(2):207-253, 1984.

[Dï4] Max Robert Dörner. The Space of Contact Forms Adapted to an Open Book. 2014. Thesis (Ph.D.)Universität zu Köln.

[DL02] Jan Denef and François Loeser. Lefschetz numbers of iterates of the monodromy and truncated arcs. Topology, 41(5):1031-1040, 2002.

[DS94] Stamatis Dostoglou and Dietmar Salamon. Self-dual instantons and holomorphic curves. Ann. of Math. (2), 139(3):581-640, 1994.

[Fis11] Joel Fish. Target-local Gromov compactness. Geom. Topol., 15(5):765-826, 2011.

[Gir02] Emmanuel Giroux. Géométrie de contact: de la dimension trois vers les dimensions supérieures. In Proceedings of the International Congress of Mathematicians, Vol. II (Beijing, 2002), pages 405-414, Beijing, 2002. Higher Ed. Press.

[GS90] Victor Guillemin and Shlomo Sternberg. Symplectic techniques in physics. Cambridge University Press, Cambridge, second edition, 1990.

[Gut13] Jean Gutt. Generalized Conley-Zehnder index. arXiv:1307.7239, pages 1-25, 2013.

[Hir64] Heisuke Hironaka. Resolution of singularities of an algebraic variety over a field of characteristic zero. I, II. Ann. of Math. (2) 79 (1964), 109-203; ibid. (2), 79:205-326, 1964.

[Hut02] Michael Hutchings. Lecture notes on Morse homology (with an eye towards Floer theory and pseudoholomorphic curves). https://math.berkeley.edu/ hutching/, pages 1-63, 2002.

[McL12a] Mark McLean. The growth rate of symplectic homology and affine varieties. Geom. Funct. Anal., 22(2):369-442, 2012.

[McL12b] Mark McLean. Symplectic homology of Lefschetz fibrations and Floer homology of the monodromy map. Selecta Math. (N.S.), 18(3):473-512, 2012.

[McL16] Mark McLean. Reeb orbits and the minimal discrepancy of an isolated singularity. Invent. Math., 204(2):505-594, 2016.

[Mil68] John Milnor. Singular points of complex hypersurfaces. Annals of Mathematics Studies, No. 61. Princeton University Press, Princeton, N.J.; University of Tokyo Press, Tokyo, 1968.

[MS98] Dusa McDuff and Dietmar Salamon. Introduction to symplectic topology. Oxford Mathematical Monographs. The Clarendon Press Oxford University Press, New York, second edition, 1998.

[MS04] Dusa McDuff and Dietmar Salamon. J-holomorphic curves and symplectic topology, volume 52 of American Mathematical Society Colloquium Publications. American Mathematical Society, Providence, RI, 2004.

[MTZ14a] Mark McLean, Mohammad Farajzadeh Tehrani, and Aleksey Zinger. Altering symplectic manifolds by homologous recombination. arxiv.org/abs/1410.2573, pages 1-52, 2014.

[MTZ14b] Mark McLean, Mohammad Farajzadeh Tehrani, and Aleksey Zinger. Normal Crossings Divisors and Configurations for Symplectic Topology. arxiv.org/abs/1410.0609, pages 1-66, 2014.

[Mus12] Mircea Mustaţă. IMPANGA lecture notes on log canonical thresholds. In Contributions to algebraic geometry, EMS Ser. Congr. Rep., pages 407-442. Eur. Math. Soc., Zürich, 2012. Notes by Tomasz Szemberg.

[Pal66] Richard S. Palais. Homotopy theory of infinite dimensional manifolds. Topology, 5:1-16, 1966.

[Pet16] Dan Petersen. A spectral sequence for stratified spaces and configuration spaces of points. arXiv preprint arXiv:1603.01137, 2016.

[RS93] Joel Robbin and Dietmar Salamon. The Maslov index for paths. Topology, 32(4):827-844, 1993.

[Sei00] Paul Seidel. Graded Lagrangian submanifolds. Bull. Soc. Math. France, 128(1):103-149, 2000.

[Sei01] Paul Seidel. More about vanishing cycles and mutation. In Symplectic geometry and mirror symmetry (Seoul, 2000), pages 429-465. World Sci. Publ., River Edge, NJ, 2001.

[Sei14] Paul Seidel. Critical points of complex polynomials from the symplectic viewpoint (lecture. joint mathematics meetings). 2014.

[Ste99] Norman Steenrod. The topology of fibre bundles. Princeton Landmarks in Mathematics. Princeton University Press, Princeton, NJ, 1999. Reprint of the 1957 edition, Princeton Paperbacks. 
[SZ92] Dietmar Salamon and Eduard Zehnder. Morse theory for periodic solutions of Hamiltonian systems and the Maslov index. Comm. Pure Appl. Math., 45(10):1303-1360, 1992.

[Ulj14] Igor Uljarevic. Floer homology of automorphisms of liouville domains. pages 1-30, 2014, arXiv:1404.2128.

[Var82] Alexander Varchenko. Contact structures and isolated singularities. Mosc. Univ. Math. Bull., 35(2):18-22, 1982.

[Vit99] Claude Viterbo. Functors and computations in Floer homology with applications. I. Geom. Funct. Anal., 9(5):985-1033, 1999.

[Zar72] Oscar Zariski. Some open questions in the theory of singularities. Uspehi Mat. Nauk, 27(5(167)):5968, 1972. Translated from the English (Bull. Amer. Math. Soc. 77 (1971), 481-491) by L. R. Gorodeckilu, A series of articles on the theory of singularities of smooth mappings. 